\title{
The geometric phase in nonlinear frequency conversion
}

\author{
Aviv Karnieli $\mathbb{1}^{1}$, Yongyao Li $\mathbb{D}^{2}$, Ady Arie $\mathbb{\circledR}^{3, \dagger}$ \\ ${ }^{1}$ Raymond and Beverly Sackler School of Physics and Astronomy, Tel Aviv University, Ramat Aviv 69978, Tel Aviv, Israel \\ ${ }^{2}$ School of Physics and Optoelectronic Engineering, Foshan University, Foshan 528000, China \\ ${ }^{3}$ School of Electrical Engineering, Fleischman Faculty of Engineering, Tel Aviv University, Tel Aviv, Israel \\ Corresponding author. E-mail: ${ }^{\dagger}$ ady@tauex.tau.ac.il \\ Received May 21, 2021; accepted June 24, 2021
}

The geometric phase of light has been demonstrated in various platforms of the linear optical regime, raising interest both for fundamental science as well as applications, such as flat optical elements. Recently, the concept of geometric phases has been extended to nonlinear optics, following advances in engineering both bulk nonlinear photonic crystals and nonlinear metasurfaces. These new technologies offer a great promise of applications for nonlinear manipulation of light. In this review, we cover the recent theoretical and experimental advances in the field of geometric phases accompanying nonlinear frequency conversion. We first consider the case of bulk nonlinear photonic crystals, in which the interaction between propagating waves is quasi-phase-matched, with an engineerable geometric phase accumulated by the light. Nonlinear photonic crystals can offer efficient and robust frequency conversion in both the linearized and fully-nonlinear regimes of interaction, and allow for several applications including adiabatic mode conversion, electromagnetic nonreciprocity and novel topological effects for light. We then cover the rapidly-growing field of nonlinear Pancharatnam-Berry metasurfaces, which allow the simultaneous nonlinear generation and shaping of light by using ultrathin optical elements with subwavelength phase and amplitude resolution. We discuss the macroscopic selection rules that depend on the rotational symmetry of the constituent meta-atoms, the order of the harmonic generations, and the change in circular polarization. Continuous geometric phase gradients allow the steering of light beams and shaping of their spatial modes. More complex designs perform nonlinear imaging and multiplex nonlinear holograms, where the functionality is varied according to the generated harmonic order and polarization. Recent advancements in the fabrication of three dimensional nonlinear photonic crystals, as well as the pursuit of quantum light sources based on nonlinear metasurfaces, offer exciting new possibilities for novel nonlinear optical applications based on geometric phases.

Keywords nonlinear optics, quasi phase matching, holography, geometric phase, nonlinear metasurfaces, Pancharatnam-Berry phase, frequency conversion

Contents

1 Introduction

2 The geometric phase

2.1 General definition and geometric origins

2.2 The geometric phase in linear optics

3 Nonlinear geometric phase of propagating waves

3.1 Basics of nonlinear frequency conversion

3.2 Nonlinear interactions with an undepleted pump

3.3 Beam shaping and holography in nonlinear photonic crystals

3.4 Spin- $1 / 2$ dynamics

3.5 Non-adiabatic geometric phase

* This article can also be found at http://journal.hep.com. cn/fop/EN/10.1007/s11467-021-1102-9.
3.6 Adiabatic geometric phase
2

2

2

3

5

5
3.7 Light propagation in nonlinear artificial gauge fields

3.8 Fully nonlinear interaction

4 Geometric phase in nonlinear metasurfaces

4.1 A brief overview of nonlinear metasurfaces

4.2 Shaping light with nonlinear metasurfaces: Dynamic phase holography

4.3 The nonlinear Pancharatnam-Berry phase in metasurfaces: Physical principles and selection rules

4.4 Nonlinear Pancharatnam-Berry beam steering, beam shaping and holography

5 Summary and perspectives

Acknowledgements

References

(C) The Author(s) 2022. This article is published with open access at link.springer.com and journal.hep.com.cn 


\section{Introduction}

Modern optical science has borrowed many concepts from quantum mechanics, and in return, enriched the variety of theoretical and experimental platforms for studying quantum phenomena. One prominent example is the geometric phase, a concept introduced to quantum physics following the work by Berry [1], who later realized [2] its connection with the earlier work by Rytov and Vladimirsky in electromagnetism [3, 4] and of Pancharatnam in optics [5], and its manifestation in other physical systems [6]. Although the adiabatic geometric phase in linear optics was the first to be demonstrated $[7,8]$, its non-adiabatic counterpart received greater acclaim. Since then, the so-called Pancharatnam-Berry phase found numerous applications in optics and constitutes today a central method for wavefront shaping and manipultaion with linear optical metasurfaces [9].

Recently, the nonlinear optical geometric phase gained attention, following advances in engineering adiabatic frequency conversion in nonlinear photonic crystals and waveguides on the one hand [10], and the development of nonlinear metasurfaces [11] on the other hand. Each of these realizations offer a great promise of applications for manipulating light, based on their respective advantages. The geometric phase accumulated by propagating waves in nonlinear photonic crystals demonstrates unique properties such as high efficiency, robustness, tunability, all-optical control, and even nonreciprocity, while giving rise to nonlinear synthetic gauge fields when extended to three dimensions. The geometric phase acquired in nonlinear metasurfaces, the nonlinear counterpart of the Pancharatnam-Berry phase, offers the advantage of flat optical elements, subwavelength resolution, tunability, and intricate selection rules dictated by geometry, which can be employed for multiplexed nonlinear holography in the higher harmonics.

In this review, we discuss the recent advances in both theory and experimental realization of the geometric phase in nonlinear frequency conversion. In Section 2 we present the geometric phase in the general context, its geometric interpretation, and its different realizations in linear optics. In Section 3, we review the geometric phase for propagating waves in nonlinear bulk media. Starting from the basics of frequency conversion, we first cover the linearized interaction under the undepleted pump approximation. This system is analogous to the quantum dynamics of a spin- $1 / 2$ particle in a magnetic field, and demonstrates both adiabatic and non-adiabatic nonlinear geometric phases for light. Generalizing the analogy to spin transport in magnetic materials, we find how nonlinear artificial gauge fields can be defined, giving rise to novel topological phenomena associated with the geometric phase. Finally, we cover the fully nonlinear interaction, and show how the geometric phase retains its original properties, while demonstrating new properties that de- pend on the initial conditions of the system. In Section 4, we discuss the geometric phase in nonlinear metasurfaces, starting from the principles of operation and the selection rules, covering applications such as wavefront engineering and nonlinear holography. We conclude the review with an outlook of possible future directions in this field.

\section{The geometric phase}

Since its discovery, the geometric phase received great attention in many areas of physics, such as condensed matter [12], quantum mechanics [1], classical mechanics [13], and optics [8]. It proved to be of paramount importance for the description of fundamental phenomena such as the different variants of the quantum Hall effect $[14,15]$ and topological insulators [16], and contributed to the development of new technologies such as flat optical elements [9]. In this section, we briefly review the general concept of the geometric phase in physics and its geometric origin (for a more detailed review we refer the reader to Ref. [17]), as well as its current use in linear-optical experiments, before moving to discuss its manifestation in nonlinear optics in the following sections.

\subsection{General definition and geometric origins}

In quantum mechanics, the geometric phase can be understood as arising from the topology of the Hilbert space of a quantum system and the parameter space controlling its Hamiltonian. This geometric notion can nevertheless be cast on a simpler, classical setting. To see this, let us first consider the parallel transport of a vector $\boldsymbol{v}$ in a curved space (such as the surface of a sphere), along some closed curve $\mathcal{C}$ (parameterized by $0 \leq t \leq T$ ). We demand that this vector is moved without change in its magnitude and without rotation with respect to the surface normal as it is transported along the chosen curve $\mathcal{C}$ [18]. In flat space, this corresponds to requiring that $\partial \boldsymbol{v}=0$, where $\partial$ denotes a derivative along the curve. In curved space, this condition is not sufficient, and one must supplement the usual derivative with a connection $\mathcal{A}$ to define a covariant derivative of $\boldsymbol{v}$ along $\mathcal{C}$ such that $(\partial+\mathcal{A}) \boldsymbol{v}=0$. The connection is a geometric property of the curved space and is closely related to its curvature. The price of this generalized definition of parallel transport is that the final vector $\boldsymbol{v}(T)$ is now rotated by an angle $\gamma$ with respect to the initial vector $\boldsymbol{v}(0)$, which exactly equals the integral of the curvature along the surface enclosed by $\mathcal{C}$ [17-19]. The amount of this "failure" to reproduce the same vector after a parallel transport along a closed curve is called holonomy (or anholonomy, as coined by Berry [6]). The rotation angle $\gamma$ quantifies the holonomy of the curved space's connection, and in our context, it will be analogous to the geometric phase of physical systems (see Fig. 1).

In a famous work by Berry [1], the geometric phase for general quantum systems was derived on the basis 
of the adiabatic theorem [20]. We consider a quantum system with a Hamiltonian $H(\boldsymbol{R})$, where $\boldsymbol{R}$ denotes a parameter vector controlling the Hamiltonian. According to the adiabatic theorem, if $\boldsymbol{R}$ is varied on a time scale much slower than the energy-level separation of the spectrum of $H$, then an eigenstate of $H(\boldsymbol{R})$, denoted by $|\psi(\boldsymbol{R})\rangle$, stays an instantaneous eigenstate such that $H[\boldsymbol{R}(t)]|\psi[\boldsymbol{R}(t)]\rangle=E[\boldsymbol{R}(t)]|\psi[\boldsymbol{R}(t)]\rangle$. It turns out, that if $\boldsymbol{R}(t)$ traces a closed curve $\mathcal{C}(t)$ in parameter space such that $\boldsymbol{R}(T)=\boldsymbol{R}(0)$, then the final state $|\psi[\boldsymbol{R}(T)]\rangle$ differs from the initial state $|\psi[\boldsymbol{R}(0)]\rangle$ only by a phase term, i.e.,

$$
|\psi[\boldsymbol{R}(T)]\rangle=\mathrm{e}^{-\frac{\mathrm{i}}{\hbar} \int_{0}^{T} E[R(t)] \mathrm{d} t} \mathrm{e}^{\mathrm{i} \gamma}|\psi[\boldsymbol{R}(0)]\rangle,
$$

where the first phase factor is the dynamic phase, while the second is the geometric phase given by

$$
\gamma=\mathrm{i} \int_{\boldsymbol{R}(0)}^{\boldsymbol{R}(T)}\left\langle\psi(\boldsymbol{R})\left|\nabla_{\boldsymbol{R}}\right| \psi(\boldsymbol{R})\right\rangle \cdot \mathrm{d} \boldsymbol{R} .
$$

where the derivative $\nabla_{\boldsymbol{R}}$ is with respect to parameterspace coordinates. One then defines the so-called Berry connection, $\mathcal{A}$, via

$$
\mathcal{A}=\mathrm{i}\left\langle\psi(\boldsymbol{R})\left|\nabla_{\boldsymbol{R}}\right| \psi(\boldsymbol{R})\right\rangle
$$

and, from it, the Berry curvature, $\mathcal{B}$, as

$$
\mathcal{B}=\nabla_{\boldsymbol{R}} \times \mathcal{A},
$$

such that by applying Stokes' theorem,

$$
\gamma=\oint_{\mathcal{C}} \mathcal{A} \cdot \mathrm{d} \boldsymbol{R}=\iint_{S} \mathcal{B} \cdot \mathrm{d} \boldsymbol{S}
$$
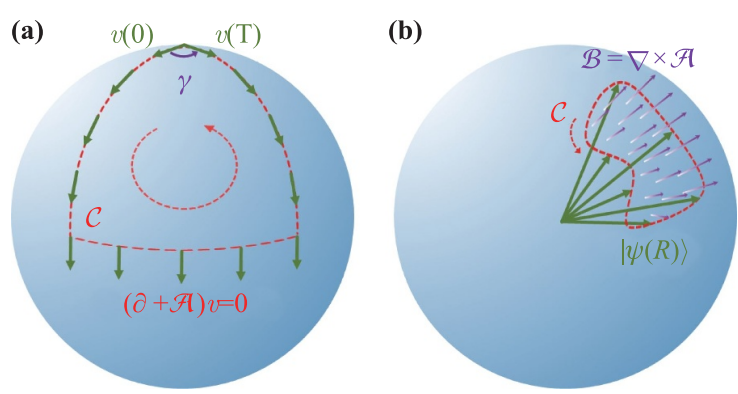

Fig. 1 The geometric phase as a parallel transport. (a) parallel transport of a vector $\boldsymbol{v}$ on the curved surface of a sphere along a closed curve $\mathcal{C}(t)$. The connection $\mathcal{A}$ provides the rule for the parallel transport. The resulting vector $\boldsymbol{v}(\boldsymbol{T})$ is rotated by an angle $\gamma$ with respect to the initial vector $\boldsymbol{v}(0)$ (the two are related via the holonomy transformation), where $\gamma$ equals the integral of the curvature over the surface enclosed by $\mathcal{C}$. (b) The geometric phase can be seen as the holonomy resulting from a parallel transport of the state vector $|\psi\rangle$ along a closed curve $\mathcal{C}$ in the projective Hilbert space. Here, the Berry connection and curvature play the role of the geometric connection and curvature. The resulting geometric phase $\gamma$ equals the surface integral of the Berry curvature $\mathcal{B}$ over the surface enclosed by $\mathcal{C}$, or alternatively by the path integral of the Berry connection $\mathcal{A}$, over the path $\mathcal{C}$. is given by the flux of the Berry curvature through the surface $S$ enclosed by $\mathcal{C}$. It is thus apparent that the Berry connection and curvature play the role of a curved space's connection and curvature, respectively, and that the geometric phase quantifies the holonomy of a generalized parallel transport of the state vector $|\psi\rangle$ along $\mathcal{C}$ [17-19] (see Fig. 1). Thus, the geometric phase, as its name suggests, is closely related to the geometrical properties of the quantum system's Hilbert space and its parameter space. For the instructive example of a spin- $1 / 2$ particle in a slowly-varying magnetic field, Berry found that the geometric phase equals half the solid angle $\Omega$ enclosed by the adiabatic trajectory of the magnetic field in its parameter space:

$$
\gamma=-\frac{1}{2} \Omega
$$

Aharonov and Anandan [21] later realized that Berry's requirement of the adiabatic rotation of $\boldsymbol{B}$ in parameter space is not necessary for the accumulation of the geometric phase, but rather, that it is acquired based on the cyclic trajectory traced by the state vector in the projective Hilbert space [18]. This observation gives rise to the notion of the non-adiabatic geometric phase. Later, Samuel and Bahandri [22] generalized the concept of the geometric phase to a more general setting, allowing noncyclic and non-unitary evolution, showing that the solid angle, determining the geometric phase, for an arbitrary curve $\mathcal{C}$ between two different points is enclosed by the shortest geodesic between these points - the so-called geodesic rule [23]. This work was based on the earlier discovery by Pancharatnam [5], who in his seminal paper discovered the geometric phase for polarized light beams, which we discuss below.

\subsection{The geometric phase in linear optics}

The geometric phase has gained tremendous attention in optics following the observation provided by Berry [2] that the adiabatic quantum mechanical geometric phase [1] and Pancharatnam's earlier, seminal work [5] on the geometric phase of polarized light beams are connected. Pancharatnam defined the phase between two polarization states on the Poincare sphere via a physical interferometric approach, and showed that this property lacks transitivity: if states $A, B$ are in-phase, and states $B, C$ are in-phase, it is not promised that states $A$ and $C$ are in-phase. By suggesting a cyclic series of projective measurements of the polarization, Pancharatnam showed that the state acquires an extra phase factor that equals half the solid angle subtended by the three points connected by the shortest geodesics between them:

$$
\gamma=-\frac{1}{2} \Omega_{A B C} .
$$

Thus Pancharatnam's phase fits more in the category of cyclic, non-unitary and non-adiabatic geometric phases, 
though it was later readily generalized to unitary and non-cyclic settings [22]. Its earliest demonstrations, using interferometry, included cyclic non-unitary evolution [24] using linear polarizers and waveplates [see Fig. 2(a)-(c)], and later a unitary evolution without polarizers [25].

Thereafter, the term Pancharatnam-Berry (PB) phase was coined, where it usually referred to the geometric phase acquired as a circularly-polarized light beam is transmitted (in parallel) through two half-waveplates at $x_{1}$ and $x_{2}$ with a relative angle $\theta$ between their fast axes [Fig. 2(d)]. Each waveplate converts the state to the opposite circular polarization, traversing different great circles (geodesics) on the Poincare sphere, with an angle of $\varphi\left(x_{2}\right)-\varphi\left(x_{1}\right)=2 \theta$ between them. The wedge traced by the two possible paths on the Poincare sphere encloses a solid angle of $\Omega=4 \theta$, thus giving $\gamma=-2 \theta$ [see Fig. 2(e)]. This observation lies at the heart of PB optical elements [26]: thin, non-refractive optical elements that received great attention in a vast variety of fields, with applications ranging from routing [26], focusing [27, 28] and guiding [29] light waves, to studies of spin-orbit coupling of light via q-plates [30-33] [Fig. 2(f)] and even quantum walks [34]. We shall discuss their nonlinear counterparts [11] in greater detail in Section 4.

The adiabatic geometric phase for light, closely resembling the setting suggested by Berry and following earlier predictions by Rytov [3] and Vladimirsky [4], was also demonstrated by Tomita and Chiao [7, 8] in linear optics using a coiled fiber [Figs. 2(g)-(i)]. In their experiment, the plane of polarization of the fiber mode was rotated as it propagated through the windings of the fiber. This corresponds to an adiabatic rotation of the wavevector $\boldsymbol{k}$ in k-space (parameter space for the Hamiltonian $H=\sigma \cdot \boldsymbol{k}$ ). Considering the photon helicity $\sigma_{p h}= \pm 1$ as a spin- 1 degree of freedom, the resulting geometric phase this time equals minus the solid angle subtended by $\boldsymbol{k}, \gamma=-\Omega$. (a)

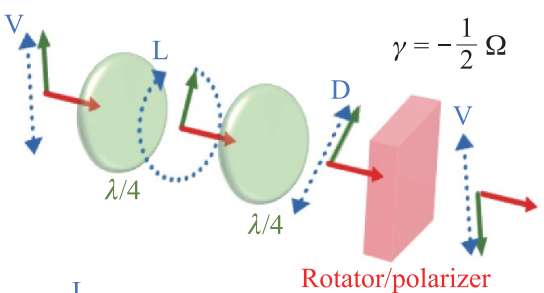

(d)

(g)

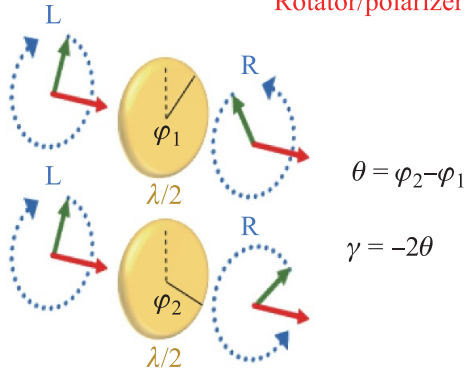

(b)

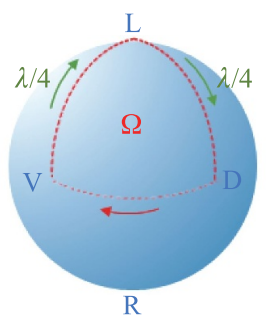

(e)

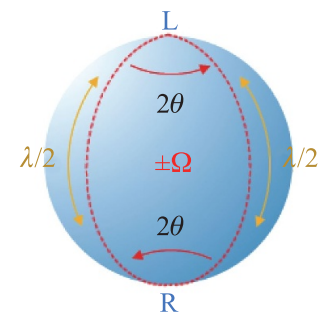

(h)

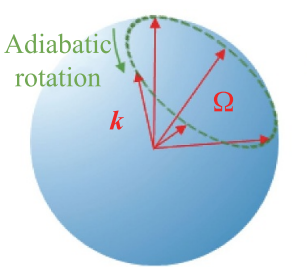

(c)

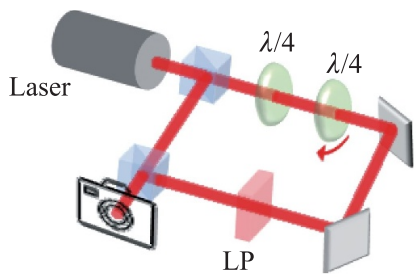

(f)

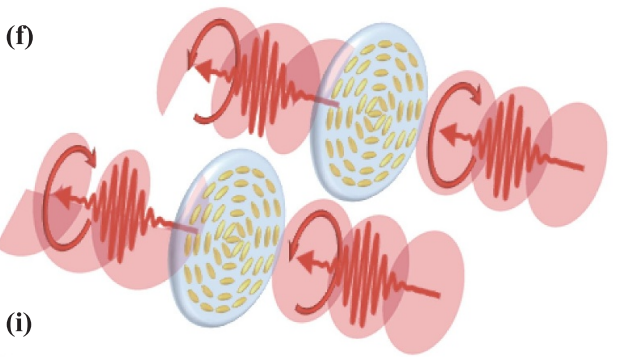

(i)

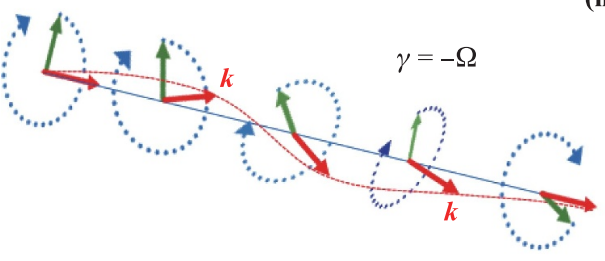

Fig. 2 The geometric phase of light in linear optics. (a-c) Pancharatnam's phase with unitary and non-unitary operations. (a) A vertical-linearly polarized (V) light beam is transmitted through a quarter-waveplate $(\lambda / 4)$, transforming it to a leftcircularly polarized (L) light beam. A second quarter-waveplate with a rotated fast-axis rotates the polarization to a diagonallinearly polarized (D) state. A linear polarizer (LP) can be used to bring the polarization state back to V, as depicted in panel (b). The resulting curve encloses a solid angle $\Omega$, where the geometric phase equals $\gamma=-\Omega / 2$. (c) Sketch of the experimental realization of Ref. [24] using a laser interferometer. (d-f) The Pancharatnam-Berry phase. (d) A left-circularly polarized (L) light beam impinges on two adjacent half-waveplates $(\lambda / 2)$, with their fast-axes having an angle $\theta=\varphi_{2}-\varphi_{1}$ between them. The state of polarization is changed to right-circular (R), but through a different geodesic along the Poincare sphere. (e) The two trajectories trace a closed curve that encloses a solid angle $\Omega=4 \theta$ and the geometric phase equals $\gamma=-2 \theta$. (f) Conversion between spin and orbital angular momentum in q-plates. A metasurface with subwavelength-patterned birefringent features of continuously varying orientation imprints the spiral Pancharatnam-Berry phase onto an incident light beam, with opposite sign for opposite polarizations. (g-i) Adiabatic geometric phase for light via rotation of the polarization plane (or equivalently, the wavevector in $\mathrm{k}$-space). (g) The propagation direction (wavevector $\boldsymbol{k}$ ) is rotated adiabatically returning to its original direction. (h) The wavevector traces a closed curve in parameter space, enclosing a solid angle $\Omega$, where now the geometric phase (associated with the photon helicity) is $\gamma=-\Omega$. (i) Depiction of the experiment by Tomita and Chiao [8] using a coiled optical fiber. 


\section{Nonlinear geometric phase of propagating waves}

The idea of geometric phase accumulation during nonlinear frequency conversion in bulk media dates back to a 1991 paper by Mandel et al. [45], which analyzed numerically the non-adiabatic geometric phase in degenerate four-wave mixing and for a degenerate parametric oscillator. Alber et al. [46] then followed with the analysis of the non-adiabatic geometric phase in nonlinear threewave mixing. Other works considered geometric phases in nonlinear bulk systems such as lasers [47] and Kerr media [48-51]. The advent of the geometrical description of nonlinear frequency conversion [52,53], as well as adiabatic frequency conversion [54-57], stimulated further research of geometric phases in nonlinear optics. Wang et al. [58] proposed a scheme for realizing a non-reciprocal, non-adiabatic geometric phase in nonlinear frequency conversion in the undepleted pump regime (the linearized interaction). Soon after, Karnieli et al. analyzed theoretically the undepleted adiabatic geometric phase in three wave mixing [59] and later observed it experimentally [60]. The analysis of the adiabatic geometric phase in the fully nonlinear regime of both three wave mixing [61] and FWM [62], soon followed. Topological effects in nonlinear gauge fields based on the geometric phase were proposed [63-65], contributing to the fast-growing field of nonlinear topological photonics [66]. In the following subsections, we review the basic concepts of nonlinear frequency conversion in bulk media and introduce the formalism needed to recover the different nonlinear geometric phases mentioned above, highlighting central results in this field.

\subsection{Basics of nonlinear frequency conversion}

The most common nonlinear interactions occur in bulk nonlinear materials, wherein the medium polarization can be written as $\boldsymbol{P}^{N L}=\epsilon_{0} \chi^{(2)} \boldsymbol{E} \boldsymbol{E}+\epsilon_{0} \chi^{(3)} \boldsymbol{E} \boldsymbol{E} \boldsymbol{E}+\ldots$ [67]. Here, $\chi^{(2)}$ and $\chi^{(3)}$ are, respectively, the second- and third-order susceptibility tensors. Parametric nonlinear processes are interactions for which the total initial and final photon energy is conserved, and can be envisioned as a virtual transition as depicted in Figs. 3(a) and (b). For second-order processes, the interaction is between three waves (hence called three-wave mixing): the idler (of frequency $\left.\omega_{i}\right)$, the pump $\left(\omega_{p}\right)$ and the signal $\left(\omega_{s}\right)$. The relation between the three frequencies is dependent on context, namely, the type of interaction considered. In sumfrequency generation (SFG), the idler and pump photons are up-converted to generate the signal photon, such that $\omega_{s}=\omega_{i}+\omega_{p}$, whereas in second harmonic generation (SHG), the idler and pump photons are identical, yielding (a)

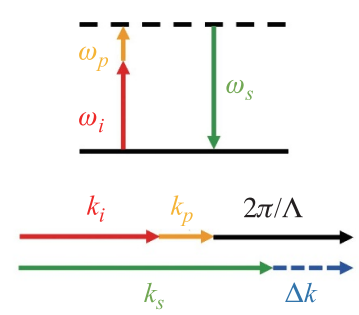

(b)

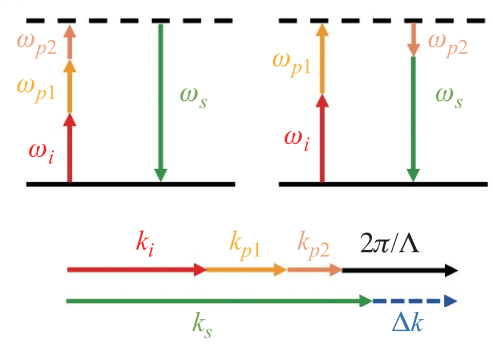

(c)

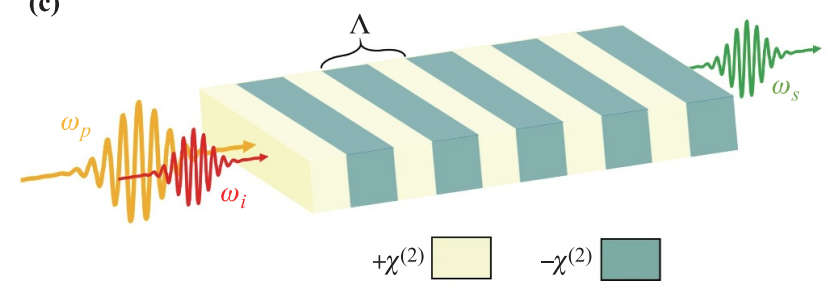

(d)

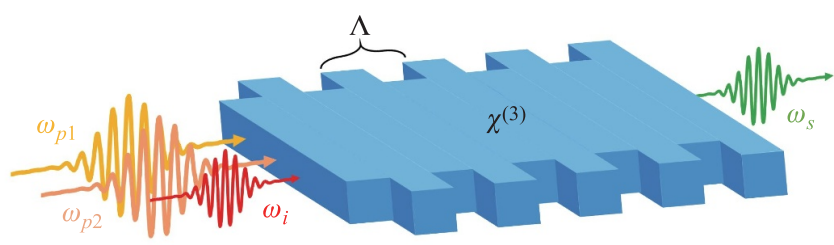

Fig. 3 Principles of nonlinear wave mixing in bulk media and waveguides. (a) In $\chi^{(2)}$ parametric interactions, energy is conserved between the idler, pump and signal photons such that $\omega_{s}=\omega_{i}+\omega_{p}$. Momentum is however not conserved, and the mismatch $\Delta k$ could be tuned via quasi-phase matching. (b) In $\chi^{(3)}$ parametric frequency conversion (four-wave mixing), we consider two possible configurations: a signal photon generated from an idler photon and two pump photons such that $\omega_{s}=\omega_{i}+\omega_{p 1}+\omega_{p 2}$, and a signal and pump photons generated from an idler and a pump photon such that $\omega_{s}+\omega_{p 2}=\omega_{i}+\omega_{p 1}$. In both cases, momentum is not conserved, and the mismatch could be compensated with quasi-phase matching. (c) Quasi phase matching of three wave mixing in nonlinear photonic crystals, where the sign of $\chi^{(2)}$ is flipped every period $\Lambda$, providing an additional momentum $2 \pi / \Lambda$ to the momentum conservation diagram. (d) Quasi phase matching of four wave mixing in corrugated $\chi^{(3)}$ waveguides with periodicity $\Lambda$, providing an additional momentum $2 \pi / \Lambda$ to the momentum conservation diagram. 
(a)

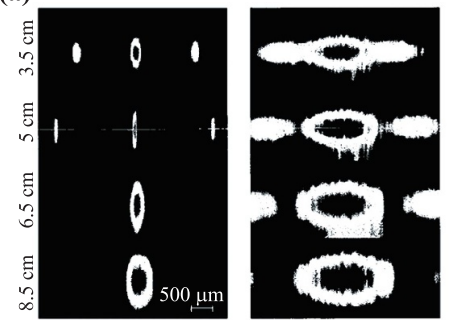

(b)

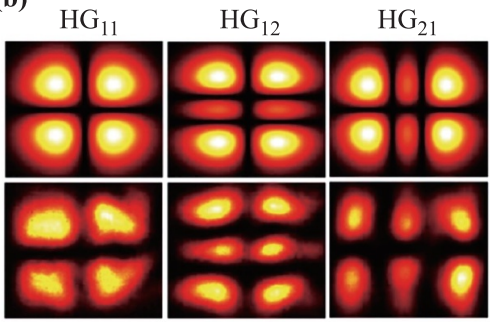

(d)

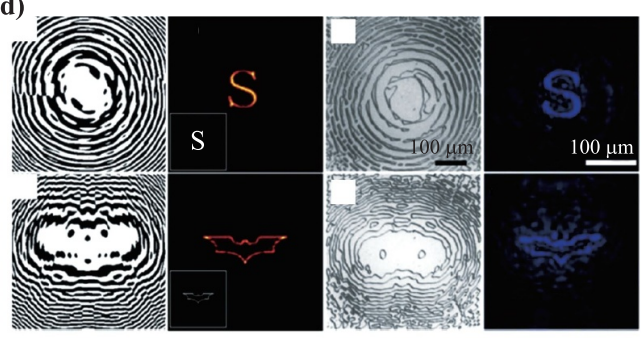

(e)

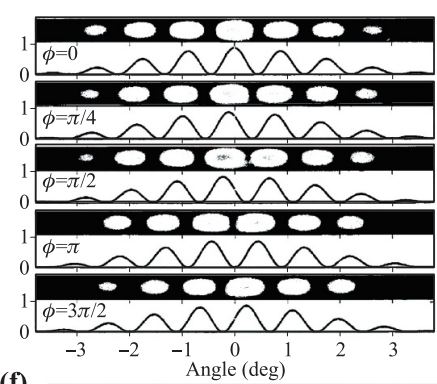

(f)

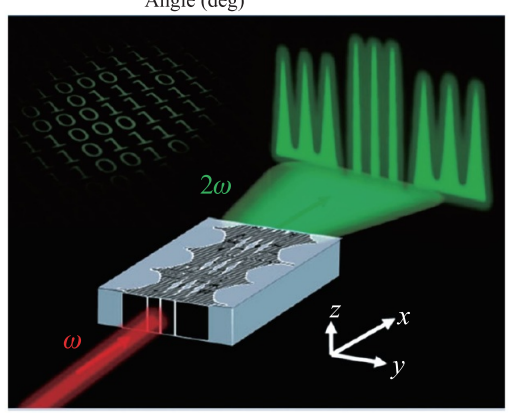

(i)

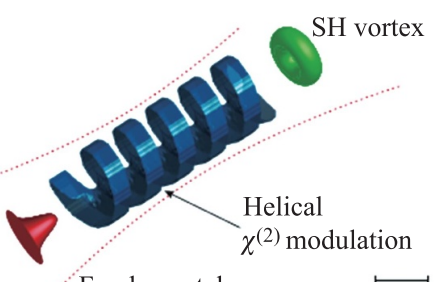

Fundamental (c)

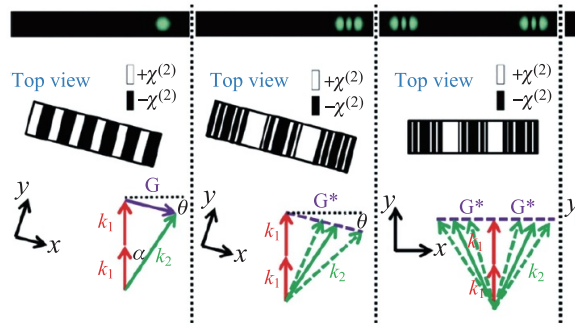

(g)
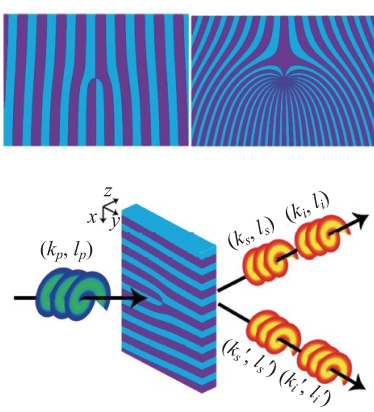

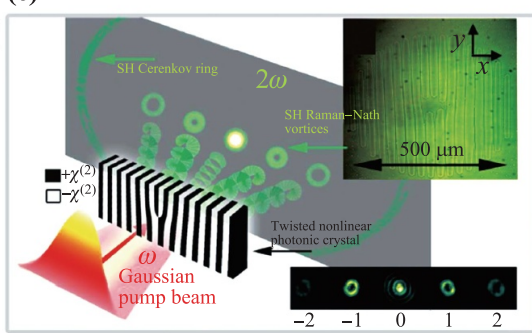

(h)
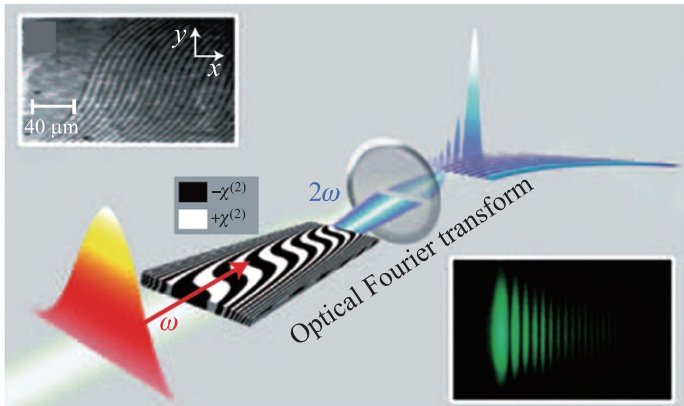

(j)
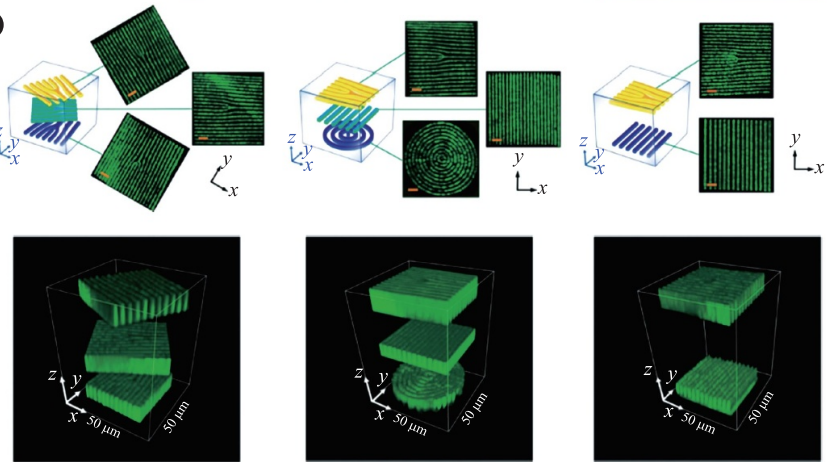

Fig. 4 Nonlinear beam shaping and holography in bulk nonlinear photonic crystals. (a) Focusing and nonlinear diffraction from 1D patterned nonlinear photonic crystals. Reproduced from Ref. [35], Copyright (C) 2002 IEEE. (b) Off-axis 2D shaping of HG beams. Reproduced from Ref. [36], Copyright () 2012 The Optical Society. (c) Off-axis symmetric and asymmetric 1D and 2D shaping of second harmonic beams. Reproduced from Ref. [37]. (d) 2D wavefront shaping and imaging of arbitrary holograms in nonlinear photonic crystals with shaped pumps. Reproduced from Ref. [38], Copyright @ 2016 The Optical Society. (e) Off-axis shaping of LG beams from fork-shaped thin 2D nonlinear crystals. Reproduced from Ref. [39], Copyright (0) 2012 American Physical Society. (f) On-axis shaping of second harmonic beams in the near-field using arbitrary holograms encoded in a 2D nonlinear photonic crystal. Reproduced from Ref. [40]. (g) Shaping spontaneously down-converted photon pairs with orbital angular momentum entanglement using vortex pump beams and fork-shaped nonlinear 2D crystals. Reproduced from Ref. [41], Copyright (C) 2015 IEEE. (h) On-axis generation of Airy beams using 2D nonlinear photonic crystals. Reproduced from Ref. [42], Copyright () 2009 Nature Group. (i) 3D design of chiral volume holograms in nonlinear waveguides. Reproduced from Ref. [43]. (j) 3D nonlinear holograms for off-axis shaping of second harmonic beams. Reproduced from Ref. [44], Copyright (C) 2019 Nature Group. 
$\omega_{s}=2 \omega_{p}$. On the other hand, in difference-frequency generation (DFG) and its quantum counterpart, spontaneous parametric down-conversion (SPDC), the pump photon is downconverted to the idler and signal photons, such that $\omega_{p}=\omega_{i}+\omega_{s}$. Third-order processes are categorized similarly. For example, four wave mixing (FWM) corresponds to the two possible up-conversion schemes depicted in Fig. 3(b), wherein $\omega_{s}=\omega_{p 1}+\omega_{p 2}+\omega_{i}$ or $\omega_{s}+\omega_{p 2}=\omega_{p 1}+\omega_{i}$. In third harmonic generation (THG) the pump frequency is tripled, i.e., $\omega_{s}=3 \omega_{p}$.

The above-mentioned processes need not, in general, conserve momentum, due to the material dispersion causing a natural finite mismatch [67] between the wave vectors: $\Delta k_{0}=\boldsymbol{k}_{s}-\boldsymbol{k}_{i}-\boldsymbol{k}_{p} \neq 0$ in SFG, or $\Delta k_{0}=$ $\boldsymbol{k}_{s}-\boldsymbol{k}_{i}-\boldsymbol{k}_{p 1}-\boldsymbol{k}_{p 2} \neq 0$ in FWM. In presence of this mismatch, the frequency conversion efficiency is hindered significantly, as the amplitude of the waves oscillates rapidly along the propagation instead of building up [67]. To mitigate this momentum mismatch, several techniques of phase matching have been employed in bulk crystals, for example ones that take advantage of birefringence [68]. In this review, we shall only consider the quasi-phase matching (QPM) technique, wherein the nonlinear coefficient is periodically modulated with period $\Lambda$ as depicted in Fig. 3(c). We note however that the geometric phase, as well as adiabatic frequency conversion, can also be accumulated in birefringently phase matched crystals by using thermal gradients [69]. In ferroelectric $\chi^{(2)}$ materials, electric field poling [70] is a standard method for QPM in one and two dimensions, wherein ferroelectric domains of the nonlinear crystal are permanently poled according to the engineered structure, thereby flipping the sign of the nonlinear coefficient [see Fig. 3(c)]. In three dimensions, relatively new techniques of laser-induced domain inversion [71] or deletion [72] are currently used. QPM $\chi^{(2)}$ crystals are commonly referred to as nonlinear photonic crystals (NLPCs). In $\chi^{(3)}$ materials, phase matching can be imposed via corrugated or tapered waveguides $[56,57]$ [Fig. 3(d)] or via intermodal phase matching [73]. In standard QPM, $\Lambda$ is chosen such that $2 \pi / \Lambda$ exactly equals the momentum mismatch. More generally, we shall denote by $\Delta k=\Delta k_{0}-2 \pi / \Lambda$ the overall momentum mismatch including the QPM contribution, which can still take a non-zero value. Figure 3 depicts the corresponding wave vector diagrams for the different QPM schemes.

For illustrating the nonlinear dynamics, we consider the common test case of three-wave mixing, though our conclusions could be generalized to FWM as well. Under the slowly varying envelope approximation [67], this dynamics is given by the set of three coupled equations:

$$
\begin{aligned}
\frac{\partial A_{1}}{\partial z} & =\mathrm{i} \frac{\chi^{(2)}(\boldsymbol{r}) \omega_{1}^{2}}{k_{1} c^{2}} A_{2}^{*} A_{3} \mathrm{e}^{-\mathrm{i} \Delta k_{0} z}, \\
\frac{\partial A_{2}}{\partial z} & =\mathrm{i} \frac{\chi^{(2)}(\boldsymbol{r}) \omega_{2}^{2}}{k_{2} c^{2}} A_{1}^{*} A_{3} \mathrm{e}^{-\mathrm{i} \Delta k_{0} z}, \\
\frac{\partial A_{3}}{\partial z} & =\mathrm{i} \frac{\chi^{(2)}(\boldsymbol{r}) \omega_{3}^{2}}{k_{3} c^{2}} A_{1} A_{2} \mathrm{e}^{\mathrm{i} \Delta k_{0} z},
\end{aligned}
$$

where $A_{1,2,3}$ are the slowly-varying envelopes of the idler, pump, and signal waves (labels for $\omega_{1}<\omega_{2}<\omega_{3}$ are given according to the frequency-conversion process under question), and $\chi^{(2)}(\boldsymbol{r})$ is the spatially varying magnitude of the second-order nonlinear susceptibility.

In the following subsections, we show how engineering of the QPM in 1D, 2D and 3D NLPCs, as well as in tapered and corrugated $\chi^{(3)}$ waveguides, can induce a fullycontrollable geometric phase imprinted on the interacting light waves. We begin by considering the linearized interaction, wherein the pump wave (or waves) is considered to be much stronger than the interacting idler and signal waves, and is thus unperturbed by their presence. This regime of undepleted pump simplifies the dynamics significantly, and renders it equivalent to the dynamics of a non-relativistic spin- $1 / 2$ quantum particle in a magnetic field, in $2 \mathrm{D}+1$ dimensions. Next, we cover the fullynonlinear interaction, wherein all the participating waves are allowed to change. We show in this case how the nonlinear geometric phase persists, while acquiring distinct new properties associated with the nonlinear dynamics.

\subsection{Nonlinear interactions with an undepleted pump}

The linearized dynamics of SFG as well as FWM, i.e., when the pump wave is considered undepleted, share a great resemblence to the dynamics of a quantum two-level system driven by an external field [20]. Without loss of generality, we consider the process of SFG in a $\chi^{(2)}$ NLPC in the undepleted pump approximation, simplifying the dynamics of Eqs. (8)-(10) to

$$
\begin{aligned}
\frac{\partial A_{i}}{\partial z} & =\mathrm{i} \frac{\chi^{(2)}(\boldsymbol{r}) \omega_{i}^{2}}{k_{i} c^{2}} A_{p}^{*} A_{s} \mathrm{e}^{-\mathrm{i} \Delta k_{0} z}, \\
\frac{\partial A_{s}}{\partial z} & =\mathrm{i} \frac{\chi^{(2)}(\boldsymbol{r}) \omega_{s}^{2}}{k_{s} c^{2}} A_{p} A_{i} \mathrm{e}^{\mathrm{i} \Delta k_{0} z} .
\end{aligned}
$$

The QPM scheme allows one to fully control the $\chi^{(2)}$ modulation structure, whereas shaping of the pump beam allows control over its wave front. The nonlinear coupling is the product of the pump field and the $\chi^{(2)}$ coefficient, and for QPM, the nonlinearity can be written as a Fourier series with slowly-varying components [59]

$$
\chi^{(2)}(\boldsymbol{r})=2 d_{i j} \sum_{m=-\infty}^{\infty} a_{m}(\boldsymbol{r}) \mathrm{e}^{\mathrm{i} m\left[\int 2 \pi / \Lambda(\boldsymbol{r}) \mathrm{d} z-\varphi_{c}(\boldsymbol{r})\right]},
$$

with $d_{i j}$ denoting the corresponding element of the nonlinear tensor [67]; $a_{m}(\boldsymbol{r})=(2 / m \pi) \sin [m \pi D(\boldsymbol{r})]$ for $m \neq 0$ and $a_{0}(\boldsymbol{r})=2 D(\boldsymbol{r})-1$, where $0 \leq D(\boldsymbol{r}) \leq 1 / 2$ denotes the modulation duty-cycle, defined as the ratio between the inverted domain length and the modulation period; and $\varphi_{c}(\boldsymbol{r})$ denotes the crystal modulation phase (see Fig. 5 for depiction of these parameters). Note that in Eq. (13), we assume that the NLPC is periodic only along the $z$ direction, with the modulation parameters $\Delta k, D$ and $\varphi_{c}$ changing slowly with respect to the QPM period $\Lambda$. How- 
ever, Eq. (13) can be easily generalized to cases where periodicity exists along more than one crystal axis.

It is common to approximate the linearized dynamical Eqs. (11) and (12) according to the rotating wave approximation, keeping only the Fourier term that is closest to phase-matching (for first-order QPM, this corresponds to the $m= \pm 1$ term). Under this approximation we find the linearized dynamics to be

$$
\mathrm{i} \frac{\partial}{\partial z}\left(\begin{array}{l}
A_{i} \\
A_{s}
\end{array}\right)=-\left(\begin{array}{cc}
0 & \kappa_{i}^{*} \mathrm{e}^{-\mathrm{i} \Phi} \\
\kappa_{s} \mathrm{e}^{\mathrm{i} \Phi} & 0
\end{array}\right)\left(\begin{array}{l}
A_{i} \\
A_{s}
\end{array}\right)
$$

where we define

$$
\Phi(\boldsymbol{r})=\int_{0}^{z} \Delta k\left(\boldsymbol{r}_{T}, z^{\prime}\right) \mathrm{d} z^{\prime},
$$

as the phase mismatch accumulated during propagation in the NLPC, and the coupling constant

$$
\kappa=2 d_{e f f} \sqrt{k_{i} k_{s}} \sin (\pi D) \mathrm{e}^{-\mathrm{i} \varphi_{c}}\left|A_{p}\right| \mathrm{e}^{\mathrm{i} \varphi_{p}}=|\kappa| \mathrm{e}^{\mathrm{i} \varphi},
$$

with $\kappa_{i, s}$ of Eq. (14) related to $\kappa$ via $\kappa_{i}=\sqrt{k_{i} / k_{s}} \kappa$ and $\kappa_{s}=\sqrt{k_{s} / k_{i}} \kappa$. In the above equation, $A_{p}(\boldsymbol{r})=$ $\left|A_{p}(\boldsymbol{r})\right| \mathrm{e}^{\mathrm{i} \varphi_{p}(\boldsymbol{r})}$ is the shaped pump field wave front (both amplitude and phase); $d_{e f f}=2 d_{i j} / \pi$; and $\varphi=\varphi_{p}-\varphi_{c}$ is the relative phase between the pump wave and the QPM modulation. All physical quantities are assumed to have a spatial dependence, as they can be continuously varied along the NLPC.

\subsection{Beam shaping and holography in nonlinear photonic crystals}

Before we introduce the concept of geometric phases in bulk nonlinear materials, it is beneficial to discuss first the well-established field of nonlinear beam shaping and holography, and then connect these previous results to the topics discussed in this review. The concept of nonlinear holography stemmed from the realization of QPM nonlinear photonic crystals, mainly by electric field poling [70] for 1D and 2D designs, and recently, with laser domain engineering [71, 72, 74-76] opening the door for $3 \mathrm{D}$ designs. Since in the QPM technique, the nonlinearity is modulated in a binary fashion, a binary encoding of computer generated holograms [77] is employed [7880]. In general, the binary nonlinear computer-generated hologram can be encoded onto the $\chi^{(2)}$ structure in the following manner [79]

$$
\chi^{(2)}(\boldsymbol{r})=\chi^{(2)} \operatorname{sign}\left\{\cos \left[\boldsymbol{G} \cdot \boldsymbol{r}-\varphi_{c}(\boldsymbol{r})\right]-\cos [\pi D(\boldsymbol{r})]\right\},
$$

where $\boldsymbol{G}$ is a reciprocal lattice vector (a carrier wavevector, transverse or longitudinal, which is responsible for either on-axis or off-axis phase matching $), \varphi_{c}(\boldsymbol{r})$ is the crystal hologram phase, and $D(\boldsymbol{r})$ is the modulation duty cycle, where the variation in the space coordinate $\boldsymbol{r}$ can be either transverse, longitudinal, or both. The firstorder Fourier coefficient of the $\chi^{(2)}$ design will be given by $\chi^{(2)}(2 / \pi) \sin [\pi D(\boldsymbol{r})] \exp \left[\mathrm{i} \boldsymbol{G} \cdot \boldsymbol{r}-\mathrm{i} \varphi_{c}(\boldsymbol{r})\right]$, and is often responsible to the most efficient QPM. The reconstructed hologram, $\sin [\pi D(\boldsymbol{r})] \exp \left[-\mathrm{i} \varphi_{c}(\boldsymbol{r})\right]$ is obtained either in the first diffraction order, if the beam shaping is done in the Fourier plane (planar holography), or in the first longitudinal QPM order (volume holography), either in the far-field or in the near-field. In these setups, the nonlinear process under consideration is usually SHG, which, under the undepleted pump approximation and assuming firstorder QPM, can be described analytically via $[79,80]$

$$
A_{2}(\boldsymbol{r})=-\frac{2}{\pi} \chi^{(2)} \frac{\mathrm{i} \omega L}{n_{2} c}\left|A_{1}(\boldsymbol{r})\right|^{2} \sin [\pi D(\boldsymbol{r})] \mathrm{e}^{\mathrm{i}\left[2 \varphi_{1}(\boldsymbol{r})-\varphi_{c}(\boldsymbol{r})\right]},
$$

where $L$ is the crystal length. Eq. (18) suggests that nonlinear spatial holography of SHG in the perturbative regime can be expressed as the product of the pump wave envelope squared $A_{1}^{2}(\boldsymbol{r})$ and the hologram encoded in the crystal structure. The amplitude would then be proportional to the fundamental beam's intensity, and to the duty cycle modulation encoded in the crystal, $\sin (\pi D)$. The phase would be proportional to the relative phase between the fundamental field squared and the phase encoded in the crystal, i.e., $2 \varphi_{1}-\varphi_{c}$.

The first realizations of nonlinear beam shaping and holography were done using 1D settings. The first such experiment by Imeshev et al. [81] used an on-axis QPM scheme to generate SHG light with a truncated beam profile. Other realizations employed diffractive nonlinear 1D gratings [35, 82] [Fig. 4(a)] as well as 1D phase and amplitude patterns for deflection [83] and holographic beam shaping into HG modes [78], where the hologram was encoded on the transverse axis of the crystal. The generalization of planar diffractive holography to two dimensions soon followed, where $2 \mathrm{D}$ designs were encoded on the transverse direction of thin nonlinear crystals using electric field poling. These included the offaxis generation of 2D Hermite-Gauss and Laguerre-Gauss beams [36, 37, 39, 84-86] and nonlinear hologram imaging [87] [Figs. 4(b), (c) and (e)].

On-axis shaping using the pump wavefront was also realized for both SHG, DFG and SFG [38, 88-90], and later also by using two-dimensional nonlinear photonic crystals $[40,91,92]$, which can also be used for tailoring the wavefront in the nearfield using arbitrary holograms [40] [Fig. 4(d) and (f)]. The nonlinear holography techniques led to the realization of unique optical beams, such as Airy beams [42, 92-97] [Fig. 4(h)], parabolic beams [94], caustic beams with switchable trajectories [98], as well as novel phenomena such as the Talbot effect [99].

Nonlinear holography need not be restricted to the spatial domain, and can also be used for spectral and temporal shaping [100-104]. This is done by the modulation of the phase-mismtach vector $\Delta k$ [through local changes in 
the longitudinal QPM lattice vector $\boldsymbol{G}$ of Eq. (17)]. Further, nonlinear bulk and planar holograms can be used to engineer the biphoton wavefunction in spontaneous parametric down-conversion [Fig. 4(g)] [41, 105-110]. For more detailed reviews of $1 \mathrm{D}$ and $2 \mathrm{D}$ nonlinear holography, the reader is referred to Refs. [79, 80].

The recent advent of three dimensional nonlinear photonic crystals $[71,72,76]$ made possible the realization of 3D nonlinear volume holography [43, 44, 111-114] [Fig. 4(i)-(j) and see also Fig. 9(d)]. These advances also offer exciting new opportunities for realization of unique quantum mechanical analogies, by employing artificial gauge fields, as we discuss in more detail in Section 3.7. For a recent and detailed review of 3D nonlinear photonic crystals and their applications for nonlinear holography, we refer the reader to Ref. [112].

We conclude this subsection with the remark that, although many of the works on nonlinear holography considered the shaping of the SHG beam as described by Eq. (18), these concepts can also be employed for other processes such as SFG and DFG [89, 90, 95]. In terms of the model we developed in the previous sections, and by assuming both undepleted pump and idler input beams, the generated signal beam is given by

$$
\begin{aligned}
A_{s}(\boldsymbol{r})= & \frac{2}{\pi} \chi^{(2)} \frac{\mathrm{i} \omega_{s} L}{n_{s} c}\left|A_{p}(\boldsymbol{r})\right| \sin [\pi D(\boldsymbol{r})] \\
& \cdot \mathrm{e}^{\mathrm{i}\left[\varphi_{p}(\boldsymbol{r})-\mathrm{i} \varphi_{c}(\boldsymbol{r})\right]} A_{i}(\boldsymbol{r}),
\end{aligned}
$$

where again, the hologram amplitude would be proportional to the pump amplitude and to the duty cycle modulation, $\left|A_{p}\right| \sin (\pi D)$. Its phase would be proportional to the relative phase between the pump wave and the crystal modulation phase, i.e. to the SFG phase $\varphi=\varphi_{p}-\varphi_{c}$ that we defined in Eq. (16). Interestingly, the holographic phases of $2 \varphi_{1}-\varphi_{c}$ and $\varphi_{p}-\varphi_{c}$ of Eqs. (18) and (19), respectively, can be connected to a special case of the nonlinear geometric phase where a wedged rotation by an angle $\varphi$ on the Bloch sphere equator is induced [See Fig. 6(d) and Section 3.6]. Thus, the past demonstrations of nonlinear holography can be envisioned as the precursor to nonlinear-geometric-phase-based beam shaping, which we discuss in Section 3.6.

\subsection{Spin- $1 / 2$ dynamics}

Let us now show how the dynamics of undepleted SFG can be recast onto the form of spin- $1 / 2$ dynamics in quantum mechanics. To see this, we can transform Eq. (14) via the transformation

$$
\left(\begin{array}{l}
A_{i} \\
A_{s}
\end{array}\right)=\sqrt{N}\left(\begin{array}{cc}
\mathrm{e}^{-\mathrm{i} \Phi / 2} \sqrt{\frac{\omega_{i}}{n_{i}}} & 0 \\
0 & \mathrm{e}^{\mathrm{i} \Phi / 2} \sqrt{\frac{\omega_{s}}{n_{s}}}
\end{array}\right) \Psi=U \Psi
$$

to the rotating frame, where $N=n_{i}\left|A_{i}\right|^{2} / \omega_{i}+n_{s}\left|A_{s}\right|^{2} / \omega_{s}$ is a constant of motion due to the Manley-Rowe rela-

tions [67], finally giving:

$$
\mathrm{i} \frac{\partial}{\partial z} \Psi=-\left(\begin{array}{cc}
\Delta k / 2 & \kappa^{*} \\
\kappa & -\Delta k / 2
\end{array}\right) \Psi=-(\sigma \cdot \boldsymbol{B}) \Psi,
$$

where $\Psi=U^{\dagger}\left(A_{i}, A_{s}\right)^{\mathrm{T}}$ (with $\left.\Psi^{\dagger} \Psi=1\right)$ denotes a spinor wavefunction comprising the (scaled and rotated) idler and signal fields; $\sigma=\left(\sigma_{x}, \sigma_{y}, \sigma_{z}\right)$ is the Pauli matrix vector, with

$$
\sigma_{x}=\left(\begin{array}{ll}
0 & 1 \\
1 & 0
\end{array}\right), \sigma_{y}=\left(\begin{array}{cc}
0 & -\mathrm{i} \\
\mathrm{i} & 0
\end{array}\right), \sigma_{z}=\left(\begin{array}{cc}
1 & 0 \\
0 & -1
\end{array}\right)
$$

and where we introduced the magnetic field equivalent

$$
\boldsymbol{B}(\boldsymbol{r})=\operatorname{Re} \kappa(\boldsymbol{r}) \hat{\boldsymbol{x}}+\operatorname{Im} \kappa(\boldsymbol{r}) \hat{\boldsymbol{y}}+\frac{\Delta k(\boldsymbol{r})}{2} \hat{\boldsymbol{z}} .
$$

The linearized, non-diffracting dynamics of SFG in the rotating frame [Eq. (21)] is, therefore, equivalent to the dynamics of a spin- $1 / 2$ particle in an external magnetic field. From this analogy, it follows that the SFG dynamics could be mapped on a corresponding frequency-domain Bloch sphere [Fig. 5(a)], where the idler and signal frequencies correspond to the north and south poles, respectively. Similarly to the Poincare sphere representation, one may define the equivalent Stokes parameters using the rotated-frame spinor $\Psi$

$$
S_{i}=\Psi^{\dagger} \sigma_{i} \Psi
$$

(a)
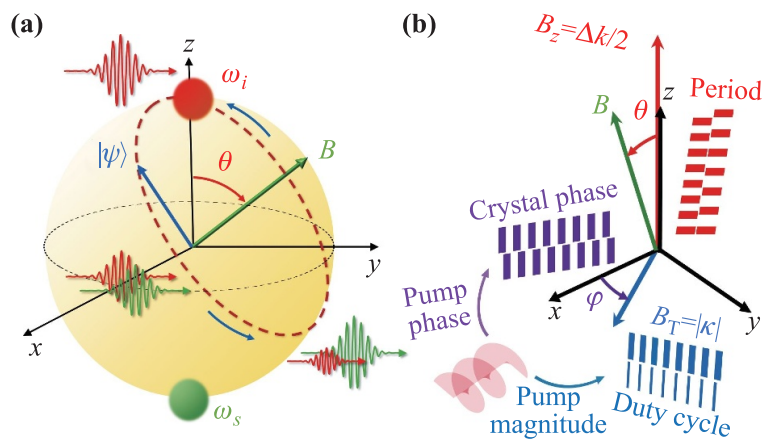

Fig. 5 Bloch sphere representation and parameter space of nonlinear frequency conversion with an undepleted pump. (a) Frequency domain Bloch sphere: the north and south poles correspond to the idler and signal frequencies, respectively. The equator corresponds to equal superpositions of idler and signal frequencies. The state vector $\Psi$ precesses around the magnetic field equivalent $\boldsymbol{B}$. (b) The parameter space of the magnetic field equivalent. The $z$-component is controlled by the momentum mismatch $\Delta k$ - namely, the poling period (marked in red). The radial component is defined by the nonlinear coupling strength $|\kappa|$, proportional to the modulation duty cycle and to the pump field strength (marked in blue). The polar angle $\varphi$ is given by the relative phase between the poling phase and pump phase front (marked in purple). $\theta$ denotes the elevation angle. All these parameters are tuneable and can be used to tailor the direction and magnitude of $\boldsymbol{B}$ at any point in space. 
or, in terms of the original field amplitudes [53]

$$
\begin{aligned}
S_{1} & =\frac{1}{N} \sqrt{\frac{n_{i} n_{s}}{\omega_{i} \omega_{s}}} 2 \operatorname{Re~e}^{-\mathrm{i} \Phi} A_{i}^{*} A_{s}, \\
S_{2} & =\frac{1}{N} \sqrt{\frac{n_{i} n_{s}}{\omega_{i} \omega_{s}}} 2 \operatorname{Im~} \mathrm{e}^{-\mathrm{i} \Phi} A_{i}^{*} A_{s}, \\
S_{3} & =\frac{1}{N}\left(\frac{n_{i}}{\omega_{i}}\left|A_{i}\right|^{2}-\frac{n_{s}}{\omega_{s}}\left|A_{s}\right|^{2}\right),
\end{aligned}
$$

with $S^{2}=S_{1}^{2}+S_{2}^{2}+S_{3}^{2}=1$. For either choice, the dynamics of the geometric state vector $\boldsymbol{S}=\left(S_{1}, S_{2}, S_{3}\right)$ can be written as a precession around the magntic field equivalent $\boldsymbol{B}$, namely

$$
\frac{\partial \boldsymbol{S}}{\partial z}=\boldsymbol{B} \times \boldsymbol{S}
$$

The Bloch sphere equator hosts equal-superpositions of the idler and signal frequencies, whereas the north and south poles represent the signal-only and idler-only states. Any other point on the surface of the sphere represents a single state vector, with different populations of the idlersignal modes (its $z$-component) and with different phases between them (azimuthal direction). For a constant $\boldsymbol{B}$ vector, say at an angle $\theta$ with respect to the $z$-axis of the Bloch sphere, and for an initial state starting at the idler frequency (north pole), the dynamics as manifested by Eqs. (21) and (26) dictates that the state vector precesses along a circle around $\boldsymbol{B}$, as depicted in Fig. 5(a) (evolution of the precession is with respect to the propagation $z$ axis). Frequency conversion is achieved as the state gets closer to the south pole. A phase-matched interaction $(\Delta k=0)$ corresponds to $B_{z}=0$ (a transverse magnetic field), such that the precession is done on a great circle passing through both poles.

The direction of the magnetic field equivalent can be determined by tuning the nonlinear interaction parameters: the NLPC modulation and the pump wave front. To see this, we rewrite the magnetic field vector in spherical coordinates as

$$
\boldsymbol{B}=B \hat{\boldsymbol{B}}(\theta, \varphi),
$$
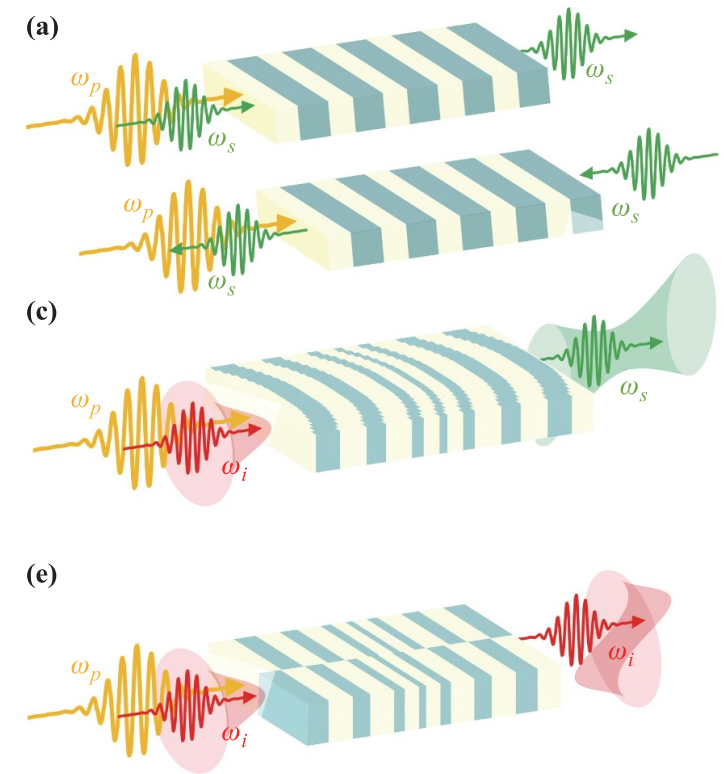
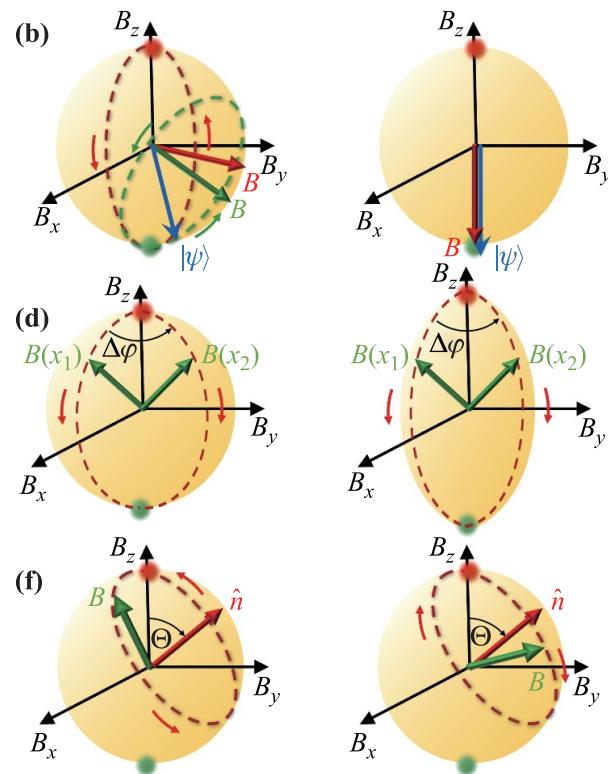

Fig. 6 Schemes of non-adiabatic and adiabatic geometric phases in nonlinear frequency conversion with an undepleted pump. (a, b) Non-adiabatic geometric phase. (a) Pump and signal beams enter a nonlinear photonic crystal, in a co-propagating (nearly phase-matched interaction) and counter-propagating (highly phase-mismatched interaction) geometry. (b) Left: Precession of the state vector $\Psi$ around $\boldsymbol{B}$ for a co-propagating interaction. The geometric phase equals half the solid-angle enclosed by the state trajectory. Right: In the counter-propagating geometry, $\Delta k \gg|\kappa|$ and therefore $\boldsymbol{B}=B_{z} \hat{\boldsymbol{z}}$, such that $\Psi$ stays parallel to $\boldsymbol{B}$ and no geometric phase is accumulated. (c, d) Non-cyclic adiabatic geometric phase on a wedge of parameter space. (c) Quadratic adiabatic geometric phase in a 2D NLPC. Pump and idler waves are incident on the engineered crystal such that different transverse positions $x_{1}, x_{2}$ correspond to a different adiabatic trajectory of $\boldsymbol{B}$, from the north pole of the parameter space surface to the south pole, thus inducing an adiabatic frequency conversion to the signal frequency. The accumulated geometric phase is quadratic, hence acting as a cylindrical lens for the signal wave. (d) Left: Spherical parameter surface. Right: Oblong surface. The extended dimension along the $z$-direction increases the robustness of the geometric phase to changes in the input frequency, while retaining the value of the geometric phase $\gamma=\Delta \varphi$. (e, f) Cyclic adiabatic geometric phase on a circle in parameter space. (e) Idler and pump beams enter an adiabatic 2D NLPC with two adjacent regions, each inducing an adiabatic circular rotation of $\boldsymbol{B}$ about a unit vector $\hat{\boldsymbol{n}}$ having an angle $\Theta$ with the $z$-axis, but with opposite orientation [panel (f)]. For a choice of $\Theta=\pi / 3$, a geometric phase difference of $\pi$ is induced between the two regions, thus approximately converting the spatial mode to $\mathrm{HG}_{10}$ in the original idler frequency. 
with $B=\sqrt{|\kappa|^{2}+(\Delta k / 2)^{2}}, \theta=\arccos (\Delta k / 2 B)$ and $\varphi$ the coupling phase defined after Eq. (16). The control over the parameters determining the spherical coordinates $B, \theta, \varphi$ is depicted in Fig. 5(b), where we also graphically illustrate the meaning of each parameter in the NLPC modulation. Namely, this is the parameter space of the Hamiltonian governing the dynamics in Eq. (21).

\subsection{Non-adiabatic geometric phase}

The revolution of the state vector $\Psi$ about a fixed magnetic field equivalent $\boldsymbol{B}$ encloses a solid angle $\Omega$ on the Bloch sphere, that is dependent on the chosen elevation angle coordinate $\theta$ [Fig. $6(\mathrm{a})]$. This solid angle equals to $\Omega=2 \pi(1-\cos \theta)$, and, much like the linear PB phase, the accumulated geometric phase along one cycle becomes

$$
\gamma=-\pi(1-\cos \theta)
$$

where it can be shown that the dynamic phase is given by $\phi_{d}=\Phi[58]$.

The nonlinear interaction possesses an inherent linear nonreciprocity attributed to the pump field bias: copropagation of the signal (idler) waves with the pump field results in a phase-matched (or nearly-phase-matched) interaction, giving the anticipated precession of $\Psi$ about $\boldsymbol{B}$; however, if the signal (idler) wave is counter-propagating with respect to the pump field, the interaction is highly mismatched, implying that $\boldsymbol{B}=B_{z} \hat{\boldsymbol{z}}$ and that $\Psi$ remains parallel to $\boldsymbol{B}$ [see Figs. 6(a)-(b)]. This property was proposed [115] and later realized experimentally [116] as a new platform of optical isolation. By extension, the nonlinear geometric phase need also demonstrate such nonreciprocity [58-60], which can be employed for nonreciprocal spatial mode conversion.

Wang et al. [58] derived the non-reciprocal, nonadiabatic geometric phase also for an arbitrary non-closed trajectory, wherein the circuit is closed by the shortest geodesic (great circle) on the Bloch sphere between the start and end points. A closed form solution could be found as function of the propagation coordinate:

$$
\gamma(z)=\arg \left\{[\cos (B z)+\mathrm{i} \cos \theta \sin (B z)] \mathrm{e}^{-\Delta k z / 2}\right\},
$$

which recovers Eq. (28) for a full cycle at every point $z=$ $m \pi / B, m=1,2, \cdots$. The results of their analysis are presented in Figs. $7(\mathrm{a})-(\mathrm{c})$.

\subsection{Adiabatic geometric phase}

Adiabatic frequency conversion [54-57] relies on the adiabatic theorem, stating that for a Hamiltonian with separate energy levels and for a slow change in its parameters, an initial eigenstate will stay an instantaneous eigenstate of the Hamiltonian. Broadband and robust frequency conversion is achieved when the magnetic field equivalent $\boldsymbol{B}$ starting, for example, at the north pole, is slowly varied such that its final orientation points at the south pole.
This ensures that the initial idler frequency eigenstate follows the variation in $\boldsymbol{B}$ such that the state ends at the signal frequency, ideally with minimal level-crossing. For this purpose, it is sufficient to change the value of $\boldsymbol{B}$ between a large (positive) value of $B_{z}$ to a large (negative) value $-B_{z}$. This can be done by introducing a linear chirp to the momentum mismatch $\Delta k(z)$ (or modulation period $\Lambda$ ), ensuring that it changes its sign once along the crystal. Another alternative that can be realized in birefringently phase matched crystals is obtained by introducing a thermal gradient $[69,117]$. A broadband efficiency is achieved since any deviation from the nominal wavelengths introduces a small change in $\Delta k$, which shifts the origin of parameter space up or down with respect to the $z$ axis. As long as the adiabatically-changing $\boldsymbol{B}$ passes through the shifted origin, high conversion efficiency is assured. This concept is illustrated in Fig. 6(d) and Fig. 7(d) [53]. For a review on adiabatic frequency conversion, we refer the reader to Ref. [10].

An adiabatic variation of the magnetic field equivalent $\boldsymbol{B}$ can also induce an adiabatic geometric phase when its trajectory encloses a solid angle in parameter space. The full controllability over the 3D orientation of the parameter vector along the NLPC is possible thanks to the tunable QPM and pump field parameters [Fig. 5]. This effect was theoretically analyzed in Ref. [59], where two possible adiabatic rotation schemes were proposed: First, a wedge scheme [Fig. 6(d) and Fig. 7(g)], where different points $x_{1}, x_{2}$ along the 2D NLPC transverse plane host adiabatically-changing magnetic fields $\boldsymbol{B}\left(x_{1}\right), \boldsymbol{B}\left(x_{2}\right)$ going from the north pole to the south pole, but with relative azimuthal phase $\Delta \varphi$ between them. Much like the PB phase, this case converts the light to the opposite (signal) eigenstate with a geometric-phase-shaped wave front wherein

$$
\gamma=-\Delta \varphi \text {. }
$$

This trajectory need not be restricted to the face of a sphere, and induces the same geometric phase for any rotationally-symmetric surface, for example an ellipsoid ellongated along the $z$-direction of parameter space. Much like the broadband response of adiabatic frequency conversion, this elongated shape ensures that the geometric phase is topologically robust against changes in input wavelength as long as the origin of parameter space remains inside the surface. For opposite wavelengths or for reversal of the crystal orientation, the phase gains an opposite sign, implying that the interaction has asymmetric transmission properties. This effect was later observed experimentally [60], using a 2D NLPC with a quadratic transverse variation in $\gamma$ acting as a geometric-phase cylindrical lens. For opposite eigenstates and opposite crystal orientations, the lens changed from converging to diverging, confirming the properties of the nonlinear geometric phase [Fig. 6(c) and Figs. 8(a, b)]. Elongating the parameter-space surface indeed resulted in a broadband efficiency [60] [Fig. 6(d)]. 
The second type of adiabatic trajectory is a circular rotation of $\boldsymbol{B}$ about some direction $\hat{\boldsymbol{n}}$ having an angle $\Theta$ with the $z$-axis of parameter space [Figs. 6(f), $7(\mathrm{f})$ and $8(\mathrm{c})]$. In this rotation, $\boldsymbol{B}$ starts and ends in the same pole, thus the output optical frequency is the same as the input one. If $\boldsymbol{B}$ rotates on the surface of a sphere, the induced geometric phase takes the usual form

$$
\gamma=-\pi(1-\cos \Theta) \text {. }
$$

For oppositely-oriented circular trajectories (right- or lefthanded), the phase gains an opposite sign. This property can be utilized to measure the geometric phase ef- fect by placing two oppositely-oriented trajectories next to one another, and observing a phase contrast [Figs. 6(e, f) and $8(\mathrm{c}, \mathrm{d})]$. This effect was also demonstrated experimentally by two adjacent opposite trajectories with $\Theta=\pi / 3$, inducing $\gamma= \pm \pi / 2$. This $\pi$ geometric phase step between the two regions of the NLPC converted the spatial mode from HG00 to (approximately) HG01, while keeping the original input frequency. Together with the linear nonreciprocity of nonlinear frequency conversion [116], this geometric phase effect realizes a nonreciprocal spatial mode converter, as implied by turning the pump wave on and off [Fig. 8(d)].

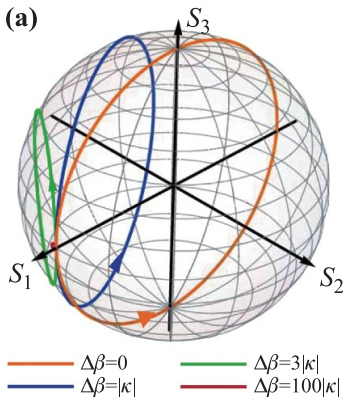

(d)
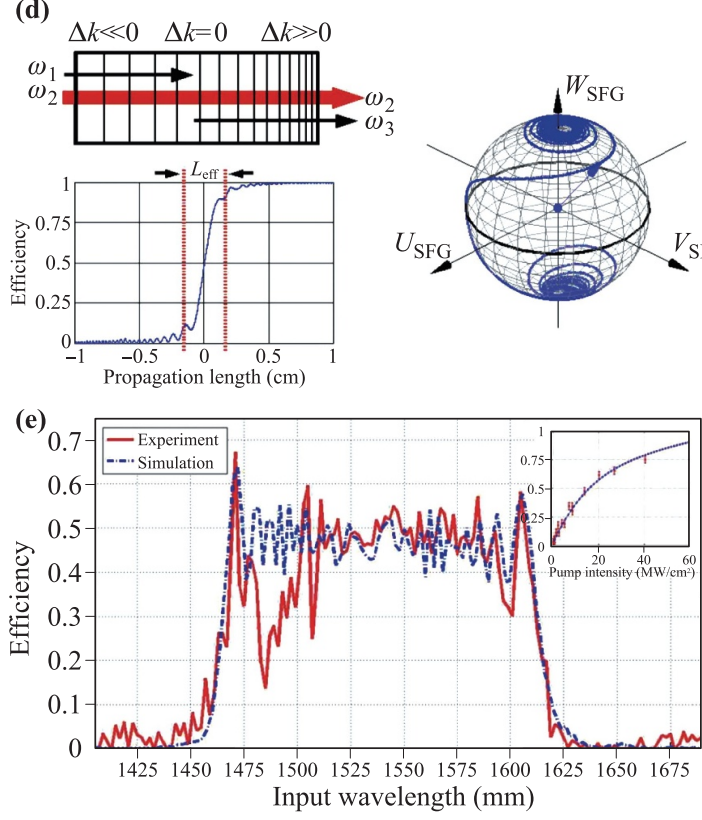

(b)

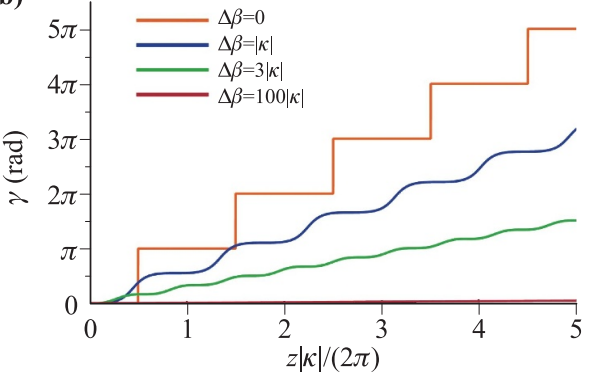

(c)

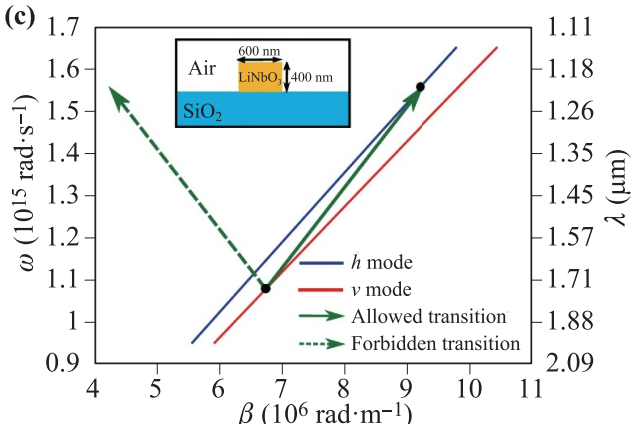

(f)
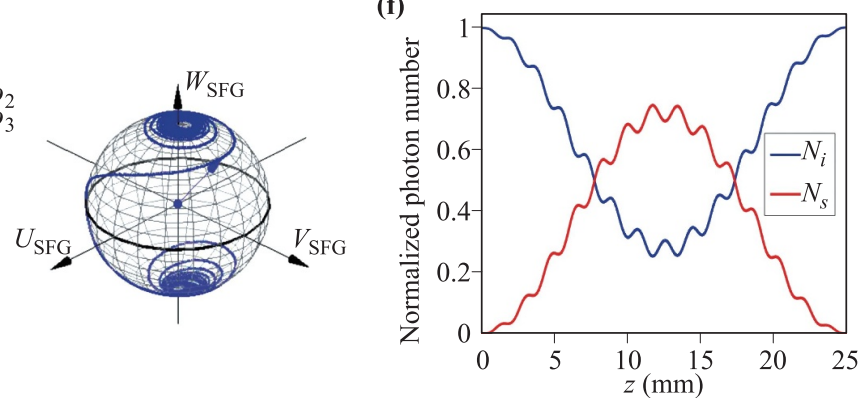

(g)

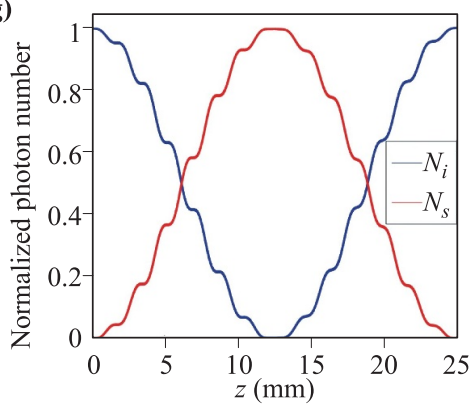

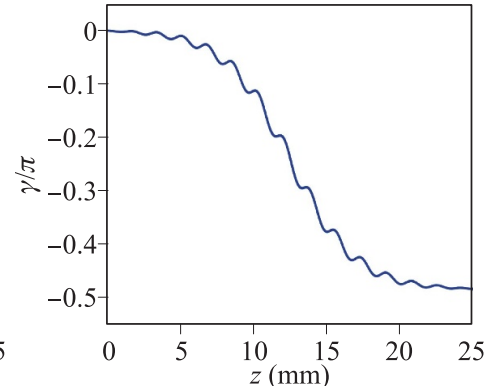

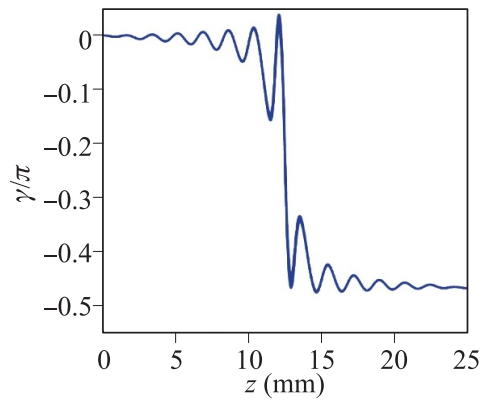

Fig. 7 Geometric phases and adiabatic frequency conversion in sum-frequency generation. (a-c) Theoretical analysis of the non-adiabatic geometric phase. (a) Circular trajectories of $\Psi$ on the Bloch sphere and (b) accumulated geometric phase for different values of momentum mismatch (here denoted as $\Delta \beta$ ). (c) Proposal for a nonreciprocal frequency conversion and geometric phase accumulation in $\mathrm{LiNbO}_{3}$ waveguides. (a-c) Reproduced from Ref. [58], Copyright () 2017 The Optical Society. (d, e) Adiabatic frequency conversion: theory and experiment. (d) an adiabatic frequency converter comprises a 1D NLPC with a chirped momentum mismatch $\Delta k$, inducing a linear adiabatic variation in $\boldsymbol{B}$ from $+B_{z}$ to $-B_{z}$. The state follows this adiabatic variation on the Bloch sphere, with a distinct step-like conversion along the propagation axis. (e) Measured conversion efficiency. The process is broadband thanks to its topological robustness: as long as the parameterspace variation of $\boldsymbol{B}$ passes through the origin (which may be shifted in the $z$-direction for different input wavelengths), high conversion efficiency is promised. (d, e) Reproduced from Ref. [53]. (f, g) Simulation of adiabatic geometric phase accumulation in an engineered 1D NLPC. (f) Left: Frequency conversion. Right: Geometric phase accumulation for a circular adiabatic trajectory [corresponding to Fig. 6(f)]. (g) Left: frequency conversion. Right: Geometric phase accumulation for a wedge adiabatic trajectory [corresponding to Fig. 6(d)]. (f, g) Reproduced from Ref. [59]. 


\subsection{Light propagation in nonlinear artificial gauge fields}

The emergence of topological photonics [118, 119] stimulated many research directions, motivated by the emulation of condensed matter systems as well as by discovery of unique topological effects in photonics. In these systems, arrays of waveguides or resonators are used to realize topologically-nontrivial band structures. Some of these systems possess a Berry phase accumulated when the light encloses a closed path in momentum space, or in another form, gains a hopping phase when it completes a circuit along discrete lattice sites. The Berry connection associated with the band structure (or hopping phase) is commonly seen as an artificial gauge field, whereas the Berry curvature acts as an artificial magnetic field. In this picture, the photonic analogs of quantum Hall and quantum spin-Hall effects could be understood as emergent from this artificial magnetism. The fast-growing comple- mentary field of nonlinear topological photonics [66] aims to resolve the effects of optical nonlinearity on topological phenomena. Many of the related works focus on topological lasers [120,121] and Kerr nonlinearity [122], while others have further shown enhanced and robust harmonic generation which mainly stemmed from the linear topological properties of the pump wave or simultaneous pump and signal waves $[123,124]$. For a recent review on this topic, we refer the reader to Ref. [66].

Here we focus the attention on a new direction in nonlinear topological photonics that originates in the topology of 3D NLPCs [112] and pump waves in bulk materials, giving rise to novel types of artificial gauge fields that manipulate light propagation. Westerberg et al. [63] were the first to propose such an effect for a degenerate three-wave mixing in $\chi^{(2)}$ crystals as well as in Kerr nonlinear $\chi^{(3)}$ materials. Such systems are inherently non-Hermitian, and their dynamics differs from Eq. (21), although many of the geo- (a)
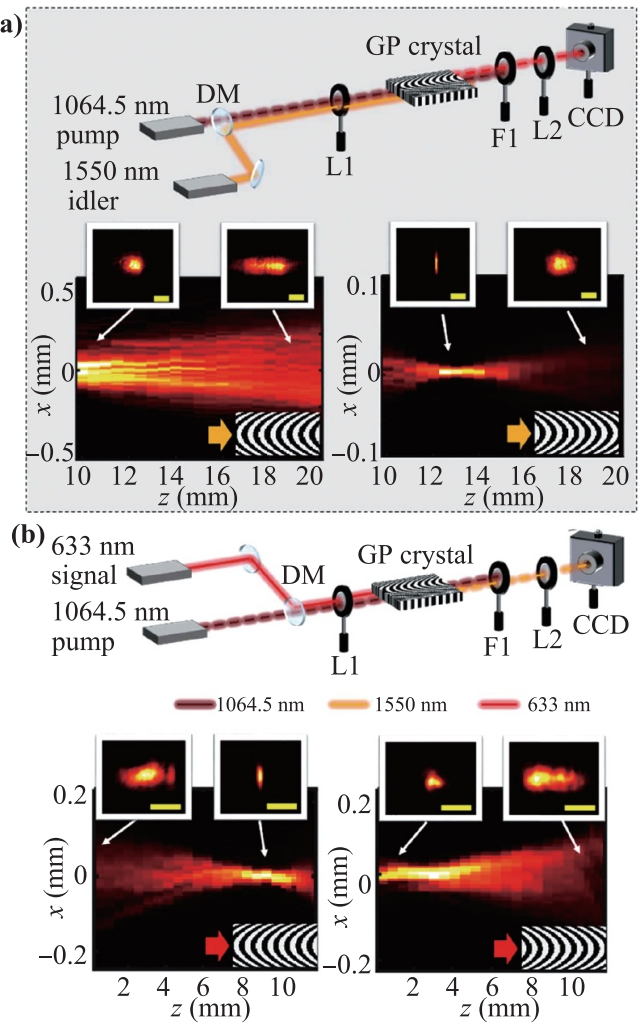

(c)
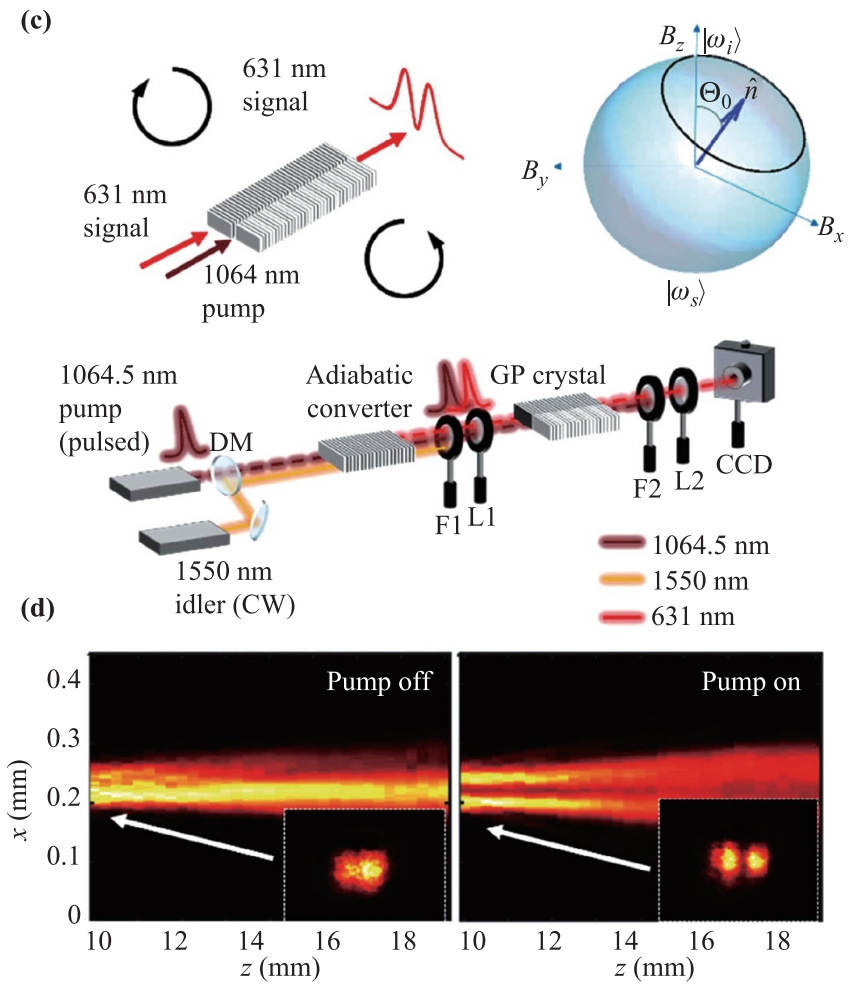

Fig. 8 Observation of the adiabatic geometric phase of sum frequency generation in 2D nonlinear photonic crystals. The experiments were performed using a $10 \mathrm{~mm}$ engineered $\mathrm{LiNbO}_{3}$ crystal (interacting wavelengths listed in the figure). (a, b) Quadratic adiabatic geometric phase along a wedge trajectory: geometric phase lens. The concept depicted in Figs. 6(c) and (d) was experimentally realized, and a cylindrical wavefront with opposite sign (converging or diverging) was observed in the converted wavelength. The lens changes from positive to negative depending on the input frequency (idler or signal) and orientation of the adiabatic trajectory (crystal direction), as expected from the geometric phase. (c, d) Adiabatic geometric phase along a circular rotation: spatial mode converter. (c) Experiment setup corresponding to the concept depicted in Fig. 6(e). An additional adiabatic converter was used before the geometric phase crystal to efficiently generate a signal pulse arriving synchronously with the pump pulse. (d) Spatial mode conversion in the original signal frequency. Left: Pump field filtered out before the geometric phase nonlinear crystal. Right: Pump field impinging on the crystal, allowing for adiabatic circular trajectories with opposite orientations as depicted in Fig. 6(f). The $\pi$ geometric phase step converts the spatial mode to $\mathrm{HG}_{10}$ while retaining the original input frequency of the signal wave. Reproduced from Ref. [60]. 
metrical properties persist. They discovered that for such interactions it is possible to apply the adiabatic limit and obtain artificial scalar and vector potentials, with $\mathcal{A}$ denoting the vector gauge field. From this gauge field they derived a synthetic magnetism that arises from the topological charge of the pump field, which was assumed to carry OAM of $\hbar l$, where $l$ is an integer. Near the center of the vortex, they found that the synthetic magnetism is

$$
\mathcal{B}=\nabla \times \mathcal{A}=-2 \alpha \hbar l \hat{\boldsymbol{z}},
$$

where $\alpha$ is a parameter capturing the strength of the magnetic field. The emergent field $\mathcal{B}$ is exactly the real-space Berry curvature associated with the accumulation of a geometric phase along propagation. For a photon fluid model, they have shown that this magnetism rotates an elliptical beam about its axis [Fig. 9(a)].

Recently, Karnieli et al. [64,65] found that these types of emergent topological phenomena are analogous to effects of spin transport in magnetic materials hosting topologically nontrivial magnetization textures, such as magnetic skyrmions $[125,126]$. To see this, we start by considering the coupled wave equations [Eqs. (11) and (12)], where we now allow diffraction under the paraxial approximation. For a pump wavelength much longer than the signal and idler wavelengths, we have that $k_{i} \sim k_{s} \sim \bar{k}$, and, applying the transformation $U$ of Eq. (20) to the rotating frame, we have that

$$
\mathrm{i} \frac{\partial}{\partial z} \Psi=\left[\frac{\left(\boldsymbol{p}_{T}-\boldsymbol{A}\right)^{2}}{2 \bar{k}}-\sigma \cdot \boldsymbol{M}(\boldsymbol{r})\right] \Psi,
$$

where $\boldsymbol{M}(\boldsymbol{r})=\boldsymbol{B}(\boldsymbol{r})$ of Eq. (21), is now seen as an analogous material magnetization (instead of an external magnetic field), and $\Psi$ now envisioned as a spinor wavefunction of a spin current, with the idler and signal frequencies (a)

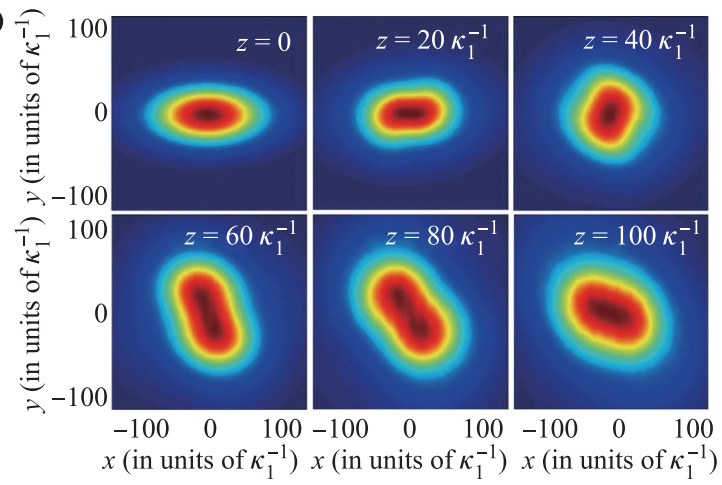

(c)
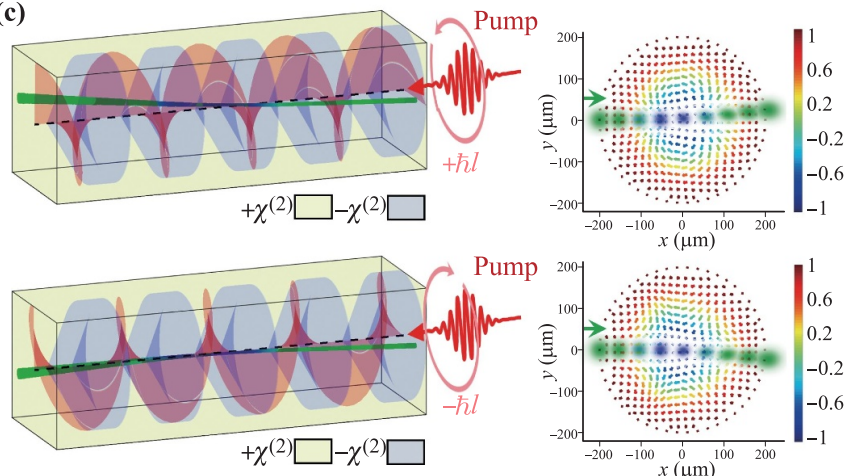

(b)

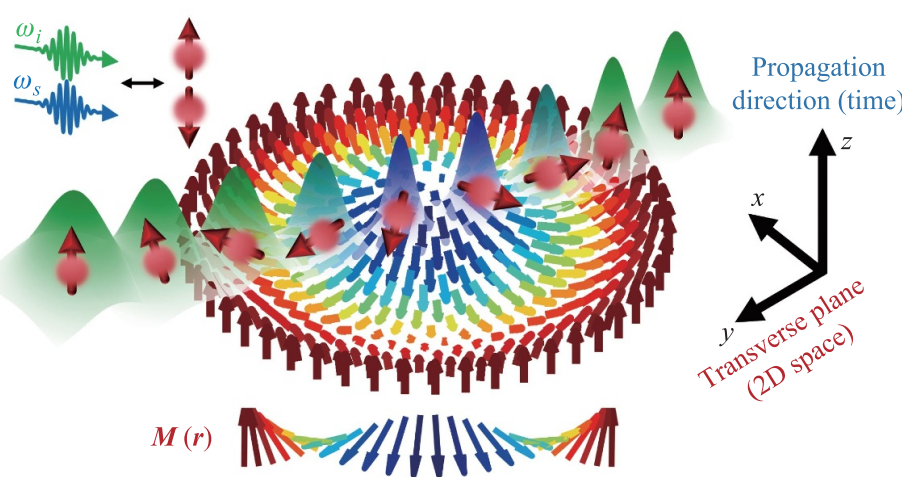

(d)

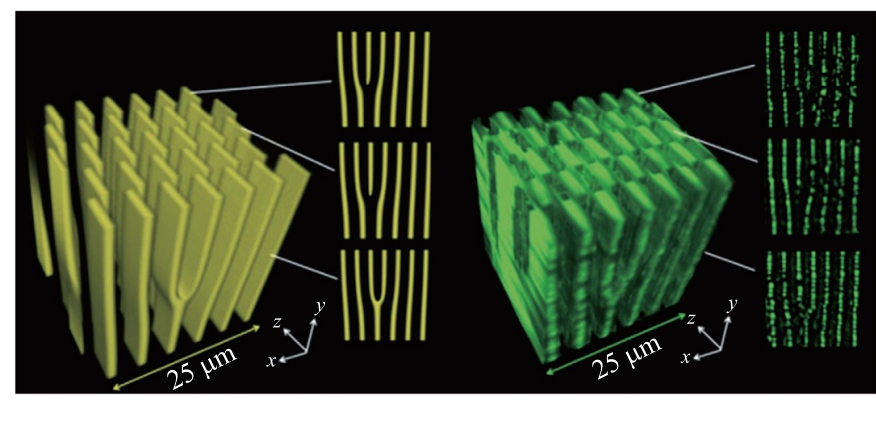

Fig. 9 Light propagation in nonlinear gauge fields. (a) Rotation of an elliptical beam about the optical axis by a pumpinduced nonlinear gauge field, giving rise to an artificial magnetic field (Berry curvature) that is proportional to the pump OAM. (a) Reproduced from Ref. [63]. (b) Concept of the topological Hall effect for light. A light beam in the idler or signal frequency (the up or down pseudospin) traverses a topologically nontrivial $\chi^{(2)}$-magnetization texture $\boldsymbol{M}(\boldsymbol{r})$, the equivalent of a magnetic skyrmion. Similarly to a $2 \mathrm{D}$ electron spinor wavefunction passing through a skyrmion, the light pseudospin follows adiabatically the local magnetization direction. The light wavefront on the transverse plane accumulates a geometric phase gradient, deflecting it with a strength proportional to the topological charge of the skyrmion. Equivalently, one can define synthetic gauge fields and artificial magnetic and electric fields that apply a Lorentz force on the light beam. (c) Proposal for an experiment to observe the topological Hall effect for light using a 3D nonlinear photonic crystal (with a radially-varying period) and a vortex pump beam of orbital angular momentum $\pm \hbar l$. The crystal and pump beams together define a nonlinear skyrmion texture, with a skyrmion number $S= \pm l$. A light beam impinging the crystal at an angle with respect to the optical axis will be deflected upwards (or downwards), depending on the skyrmion number. (b, c) Reproduced from Ref. [64]. (d) Recent advances in fabrication of volume holograms in 3D nonlinear photonic crystals. Shown is a fabricated layered fork grating for generation of vortex second-harmonic beams. (d) Reproduced from Ref. [113]. 
acting as a pseudospin degree of freedom; $\boldsymbol{p}_{T}=-\mathrm{i} \nabla_{T}$ is the transverse momentum operator with $\bar{k}$ the analog of mass; and $\boldsymbol{A}=\frac{1}{2} \nabla_{T} \Phi \sigma_{z}$ is an emergent gauge field from the transformation to the rotating frame, where $\Phi$ is defined in Eq. (15). Eq. (33), therefore, describes the dynamics of the photonic analogue of spin transport in the presence of material magnetization.

When the magnetization vector $\boldsymbol{M}(\boldsymbol{r})$ changes slowly with $\boldsymbol{r}$ such that the adiabatic approximation holds, we can decouple the dynamics of the two eigenstates pointing parallel and anti-parallel to the magnetization direction. This can be done by applying a unitary transformation $\Psi=\mathcal{U} \tilde{\Psi}$ that rotates the $z$-direction of the pseudospin to point along the local magnetization direction $\hat{M}(\boldsymbol{r})$. From this local gauge transformation $\mathcal{U}(\boldsymbol{r})$ we obtain two new artificial gauge fields, $\mathcal{A}=\mathrm{i} \mathcal{U}^{\dagger} \nabla_{T} \mathcal{U}+\mathcal{U}^{\dagger} \boldsymbol{A} \mathcal{U}$ and $\mathcal{V}=$ $-\mathrm{i} \mathcal{U}^{\dagger} \partial_{z} \mathcal{U}-M \sigma_{z}$, with a new dynamics in terms of the rotated spinor $\tilde{\Psi}$ :

$$
\mathrm{i} \frac{\partial}{\partial z} \tilde{\Psi}=\left[\frac{\left(p_{\mathrm{T}}-\mathcal{A}\right)^{2}}{2 \bar{k}}+\mathcal{V}\right] \tilde{\Psi}
$$

which, as the effective Hamiltonian is now diagonal, can be readily decoupled into two independent equations of motion for each pseudospin component of $\tilde{\Psi}$.

From the artificial gauge fields $\mathcal{A}$ and $\mathcal{V}$, one can derive emergent magnetic and electric fields as follows

$$
\begin{aligned}
& \mathcal{E}=-\nabla \mathcal{V}-\frac{\partial \mathcal{A}}{\partial z}=\frac{M_{\mathrm{T}} \nabla M_{\mathrm{T}}}{M}, \\
& \mathcal{B}=\nabla \times \mathcal{A}=-\frac{1}{2} \hat{\boldsymbol{z}}\left[\hat{\boldsymbol{M}} \cdot\left(\frac{\partial \hat{\boldsymbol{M}}}{\partial x} \times \frac{\partial \hat{\boldsymbol{M}}}{\partial y}\right)\right],
\end{aligned}
$$

with $M_{\mathrm{T}}$ denoting the transverse (in-plane) component of $\boldsymbol{M}$, and $M=|\boldsymbol{M}|$. The electric field originates in the dynamic phase and results in deflection along a gradient in the nonlinear coupling strength (since $M_{\mathrm{T}}=\kappa$ ), giving rise to an optical analogue of the Stern-Gerlach effect $[127,128]$. The emergent magnetic field $\mathcal{B}$ again serves as the real-space Berry curvature, such that a geometric phase difference is accumulated when different parts of the wavefunction $\tilde{\Psi}$ traverse different paths in real space: $\gamma=\oint \mathcal{A} \cdot \mathrm{d} l$. The emergent electric and magnetic fields exert a Lorentz-like force on the eigenstates of the system [64], $\mathcal{F}=q_{s}(\mathcal{E}+\boldsymbol{v} \times \mathcal{B})$, with $q_{s}= \pm 1$ being a pseudospin-dependent charge for the two frequencies. A gradient in the real-space geometric phase can therefore result in topological deflection of light beams.

One example of this phenomenon is the topological Hall effect [129-132], wherein polarized spin currents are deflected into opposite directions, corresponding to their spin, via interaction with a magnetic skyrmion. Skyrmions [133] are topologically nontrivial spin textures that map the 3D magnetization vector $\hat{\boldsymbol{M}}$ onto a $2 \mathrm{D}$ plane. The spatial variation in $\hat{\boldsymbol{M}}$ covers all directions on the unit sphere, and the number of times it does so is called the skyrmion number,

$$
\begin{aligned}
S & =\frac{1}{4 \pi} \iint \mathrm{d}^{2} \boldsymbol{r}\left[\hat{\boldsymbol{M}} \cdot\left(\frac{\partial \hat{\boldsymbol{M}}}{\partial x} \times \frac{\partial \hat{\boldsymbol{M}}}{\partial y}\right)\right] \\
& =-\frac{1}{2 \pi} \iint \mathcal{B} \cdot \mathrm{d} \boldsymbol{a} .
\end{aligned}
$$

The skyrmionic texture, therefore, naturally induces a real-space Berry curvature, and it can be implemented in nonlinear optics by using 3D NLPCs and shaped pump beams [see Figs. 9(b) and (c)]. In Ref. [64], it was proposed to employ the full controllability over the interaction parameters (Fig. 5) to engineer the variation in $\boldsymbol{M}(\boldsymbol{r})$ such that it realizes high-order $(S>1)$ skyrmions that are otherwise thermodynamically unstable in condensed matter systems. The topological deflection of light beams of different frequencies by such nonlinear skyrmions was numerically calculated, and was found to agree with a kinematic model where the light experiences a Lorentz force. Recent advances in the fabrication of 3D NLPCs [43, 44, 71, 72, 112-114] [Fig. 9(d) and Figs. 4(i, j)] offer a promising route towards the experimental realization of these novel phenomena in nonlinear topological photonics of bulk media.

\subsection{Fully nonlinear interaction}

When the intensities of the signal and idler waves become comparable to that of the pump wave, the undepleted pump approximation is no longer valid since the superposition principle and the conservation of the total photon number cannot be assumed anymore. Under this circumstance, frequency conversion becomes fully nonlinear. The non-adiabatic geometric phase was investigated in this regime by Alber et al. [46]. Further analysis of the geometric description of the nonlinear interaction was presented by Luther et al. [52], while a comprehensive physical model of adiabatic three-wave mixing was developed later by several researchers, for example Porat and Arie [134], Phillips et al. [135-137], Heese et al. [138-140], and Yaakobi et al. [141, 142]. In more recent works, $\mathrm{Li}$ et al. [61, 62], Lü et al. [143], and Zhao et al. [144] have shown how one can generate and control the adiabatic geometric phase in the fully nonlinear regime of both threeand four-wave mixing. Below, we detail the geometric representations of fully-nonlinear three wave mixing and its corresponding geometric phase.

We begin by defining the following dimensionless amplitudes, using similar notations as in the preceding sections:

$$
q_{j}=E_{j} \mathrm{e}^{\mathrm{i} \Phi(z)}\left(\frac{\omega_{j}}{n_{j}} \sum_{l=1}^{3} \frac{n_{l}}{\omega_{l}}\left|E_{l 0}\right|^{2}\right)^{-1 / 2},
$$

with $E_{l 0}, l=1,2,3$ standing for the field strength at $z=$ 0 , and a dimensionless propagation coordinate $\tau=\alpha z$, 
where

$$
\alpha=\frac{4 d_{i j}}{\pi c}\left(\frac{\omega_{1} \omega_{2} \omega_{3}}{n_{1} n_{2} n_{3}} \sum_{l=1}^{3} \frac{n_{l}}{\omega_{l}}\left|\tilde{E}_{l 0}\right|^{2}\right)^{1 / 2} .
$$

In addition, we define the dimensionless momentum mismatch $\Delta \Gamma=\Delta k(\tau) / \alpha$, and the dimensionless coupling $g(\tau)=\sin [\pi D(\tau)] \exp \left[i \varphi_{c}(\tau)\right]$. Substituting into Eqs. (8)-(10), they are recast into a canonical Hamiltonian structure

$$
\frac{\mathrm{d} q_{j}}{\mathrm{~d} \tau}=-2 \mathrm{i} \frac{\partial H}{\partial q_{j}^{*}},
$$

where the Hamiltonian is

$$
H=\frac{1}{2}\left(g q_{1}^{*} q_{2}^{*} q_{3}+g^{*} q_{1} q_{2} q_{3}^{*}\right)-\frac{\Delta \Gamma}{2} \sum_{j=1}^{3}\left|q_{j}\right|^{2} .
$$

The Manley-Rowe (MR) relations [67] define 3 constants of motion for this Hamiltonian, given by $K_{1}=\left|q_{1}\right|^{2}+\left|q_{3}\right|^{2}$, $K_{2}=\left|q_{1}\right|^{2}-\left|q_{2}\right|^{2}$, and $K_{3}=\left|q_{2}\right|^{2}+\left|q_{3}\right|^{2}$.

The geometric representation of the state space is represented by a set of coordinates $(X, Y, Z)$ and a surface $\varphi(X, Y, Z)=0$ on which the state vector can move. These are defined by $[52,135]$

$$
\begin{aligned}
& X+\mathrm{i} Y=q_{1} q_{2} q_{3}^{*}, \quad Z=\left|q_{3}\right|^{2}, \\
& \varphi=X^{2}+Y^{2}-Z\left(Z-K_{1}\right)\left(Z-K_{3}\right) .
\end{aligned}
$$

The shape and size of this surface are defined by the initial condition of $q_{1,2,3}$ at $\tau=0$ through the MR parameters. The Hamiltonian can be expressed via the coordinates (42) as

$$
H=\left(g_{\mathrm{r}} X+g_{\mathrm{i}} Y\right)+\Delta \Gamma\left[Z-\left(K_{1}+K_{3}\right)\right] / 2,
$$

where $g_{\mathrm{r}}=\operatorname{Re} g$ and $g_{\mathrm{i}}=\operatorname{Im} g$. Every state of the nonlinear system can be mapped onto a state surface. In the linear undepleted pump regime, this surface therefore reduces to the Bloch sphere.

Based on the geometric definition in Eqs. (42)-(44), the dynamics of Eq. (40) can be expressed in geometric form

(a)

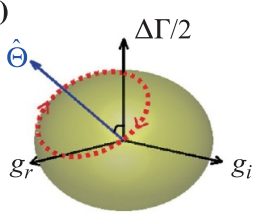

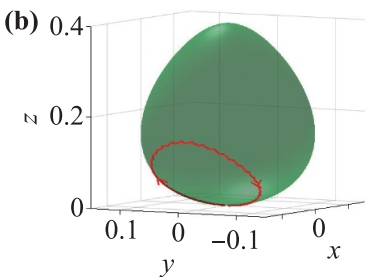

Fig. 10 (a) A typical example of a clockwise circular rotation of the QPM vector $\boldsymbol{B}(\tau)$ on its parameter space sphere. (b) A corresponding rotation of the state vector, $\boldsymbol{W}(\tau)$, which follows the clockwise rotation of the QPM vector from the bottom of the surface $\varphi=0$ and draws a trajectory on it. Reproduced from Ref. [61]. by defining a state vector $\boldsymbol{W}=(X, Y, Z)$ and an effective magnetic field vector $\boldsymbol{B}(\tau)=\nabla H=\left(g_{\mathrm{r}}, g_{\mathrm{i}}, \Delta \Gamma / 2\right)$ as

$$
\mathrm{d} \boldsymbol{W} / \mathrm{d} \tau=\{\boldsymbol{W}, H\}=\boldsymbol{B}(\tau) \times \nabla \varphi,
$$

where $\nabla=\left(\partial_{X}, \partial_{Y}, \partial_{Z}\right)$. The comparison between the geometric representations for the undepleted and depleted regimes of three-wave mixing is shown in Table 1 . The undepleted dynamics are recovered for $\left|q_{2}\right|^{2} \gg\left|q_{3}\right|^{2},\left|q_{1}\right|^{2}$, or $K_{3} \gg Z$, where the state surface becomes a sphere. With this comparison, it is obvious that we can interpret the adiabatic geometric phase by means of this geometric description, as was similarly done in the undepleted case.

As in the undepleted regime, the typical processes for depleted three-wave mixing are SFG and DFG. For SFG, the initial conditions are generally set as $q_{1,2}^{(0)} \neq 0$ and $q_{3}^{(0)}=0$, which indicates that $\boldsymbol{W}$ is initiated at the south pole of the state surface. For DFG, we can set the initial conditions to be $q_{2,3}^{(0)} \neq 0$ and $q_{1}^{(0)}=0$. In this case, $\boldsymbol{W}$ is initiated at the north pole of the state surface. We now consider a cyclic adiabatic evolution of the magnetic field equivalent $\boldsymbol{B}$, such that $\boldsymbol{W}$ remains its instantaneous

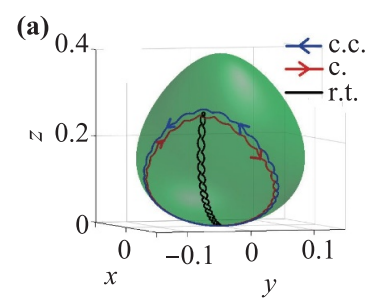

(c)

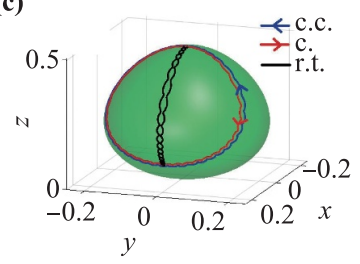

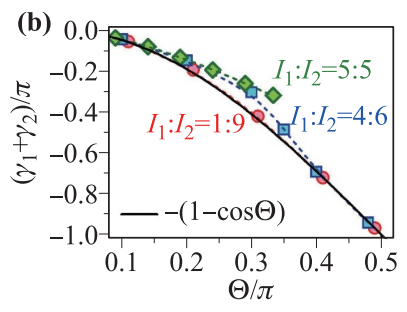

(d)

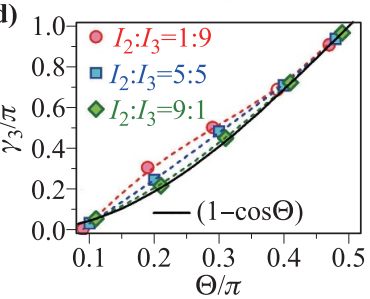

Fig. 11 Typical examples for the calculation of the AGP in the process of SFG $(\mathbf{a}, \mathbf{b})$ and DFG $(\mathbf{c}, \mathbf{d})$ when the magnetic field equivalent performs a circular rotation defined in the same way as in the undepleted pump case. (a) Circular rotation for SFG. The blue and the red trajectories show the counterclockwise (c.c.) and clockwise (c.) rotations, respectively. The black trajectory is the round-trip (r.t.) motion. Here, we select $\Theta=0.3 \pi$ and $\left(I_{1}, I_{2}, I_{3}\right)=(0.4,0.6,0)$. (b) The sum of the AGPs for $q_{1,2}$, which are calculated for the clockwise rotation, versus $\Theta$ with different depletion levels in the process of SFG. Here, the solid curves are the theoretical prediction for the undepleted case, i.e., $\gamma=-\pi(1-\cos \Theta)$. (c) Circular rotation for DFG, with $\Theta=\pi / 3$ for initial conditions of $\left(I_{1}, I_{2}, I_{3}\right)=$ $(0,0.5,0.5)$. The meaning of the curves are the same as in panel (a). (d) The AGP for $q_{3}$ (i.e., $\gamma_{3}$ ), which is also calculated from the clockwise rotation, as a function of $\Theta$ for different ratios $I_{2} / I_{3}$. The dashed curves are the theoretical predictions for the undepleted case, i.e., $\gamma=\pi(1-\cos \Theta)$. Reproduced from Ref. [61]. 
Table 1 Comparison between the geometric representation in the undepleted (linear) and depleted (nonlinear) three-wave mixing. The constants of motion $K, K_{1}$ and $K_{3}$ arise from the Manley-Rowe relations.

\begin{tabular}{lcr}
\hline & Undepleted (linear) & Depleted (nonlinear) \\
\hline Normalized field amplitudes & $\Psi=\left(q_{1}, q_{3}\right)$ & $\left(q_{1}, q_{2}, q_{3}\right)$ \\
\hline Coordinates & $S_{1}+\mathrm{i} S_{2}=2 q_{1}^{*} q_{3}$ & $X+\mathrm{i} Y=q_{1} q_{2} q_{3}^{*}$ \\
& $S_{3}=\left|q_{3}\right|^{2}-\left|q_{1}\right|^{2}$ & $\left.q_{3}\right|^{2}$ \\
\hline Bloch surface & $\varphi=S_{1}^{2}+S_{2}^{2}+\left(S_{3}-K\right)\left(S_{3}+K\right)$ & $X^{2}+Y^{2}-Z\left(Z-K_{1}\right)\left(Z-K_{3}\right)$ \\
& $\left(K=\left|E_{3}\right|^{2}+\left|E_{1}\right|^{2}\right)$ & $\boldsymbol{W}=(X, Y, Z)$ \\
\hline State vector & $\boldsymbol{S}=\left(S_{1}, S_{2}, S_{3}\right)$ & $g$ \\
\hline Coupling & $\kappa$ & $\Delta \Gamma$ \\
\hline Phase mismatch & $\Delta k$ & $\boldsymbol{B}=\nabla H=\left(g_{\mathrm{r}}, g_{\mathrm{i}}, \Delta \Gamma / 2\right)$ \\
\hline Magnetic field equivalent & $\boldsymbol{B}=(\operatorname{Re} \kappa, \operatorname{Im} \kappa, \Delta k / 2)$ & $\mathrm{d} \boldsymbol{W} / \mathrm{d} \tau=\boldsymbol{B} \times \nabla \varphi$ \\
\hline Dynamical equation & $\mathrm{d} \boldsymbol{S} / \mathrm{d} z=\boldsymbol{B} \times \boldsymbol{S}$ &
\end{tabular}

eigenstate over the entire process, and $\boldsymbol{B}(0)=\boldsymbol{B}(T)$. Figures $10(\mathrm{a})$ and (b) show a typical example for such a circular rotation of $\boldsymbol{B}$ in its parameter space and the corresponding trajectory of the state vector $\boldsymbol{W}$ on the state surface.

According to the above discussion, the variation of the amplitudes $q_{j}(j=1,2,3)$ throughout the whole process can be assumed to correspond to an instantaneous eigenstate $\tilde{q}_{j}(\tau)$ with an eigenvalue $\mu_{j}$, times a geometric phase factor, such that $q_{j}(\tau)=\tilde{q}_{j}(\tau) \mathrm{e}^{\mathrm{i} \gamma_{j}(\tau)}$, where $\gamma_{j}$ is the accumulated adiabatic geometric phase of the $j$-th wave. From the dynamical equation Eq. (40), the overall adiabatic geometric phase $\gamma_{j}$ can be obtained

$$
\begin{aligned}
\gamma_{j} & =\int_{0}^{T}\left(-\frac{1}{\left|q_{j}\right|^{2}} 2 q_{j}^{*} \frac{\partial H}{\partial q_{j}^{*}}\right) \mathrm{d} \tau-\int_{0}^{T} \mu_{j} \mathrm{~d} \tau \\
& =\Phi_{j}-\mathcal{D}_{j},
\end{aligned}
$$

where $\Phi_{j}$ and $\mathcal{D}_{j}$ are the total phase and the dynamical phase of $q_{j}$, respectively.

A direct geometric way for obtaining $\gamma_{j}$ would be to compare the phase accumulated over a trajectory of $\boldsymbol{W}$ that encloses a solid angle (due to a circular rotation of $\boldsymbol{B})$, with one that does not (a round trip trajectory): see Figs. 11(a) and (c). The round trip that we consider is exactly the vertical projection of the circular rotation, which we realize by setting $\varphi_{\mathrm{c}} \equiv 0$ (i.e., no azimuthal rotation of $\boldsymbol{B})$. Defining the phase-space variables $q_{j}^{\prime}=q_{j} / N_{j}$, $p_{j}^{\prime}=-\mathrm{i} q_{j}^{*} / N_{j}$ (where $\left.N_{j}=\sqrt{2\left|q_{j}\right|^{2}}\right)$, Eq. (46) can be represented as

$$
\gamma_{j}=\int_{\bigcirc} p_{j}^{\prime} \mathrm{d} q_{j}^{\prime}-\int_{\uparrow \downarrow} p_{j}^{\prime} \mathrm{d} q_{j}^{\prime}=\Phi_{j}^{c}-\Phi_{j}^{\mathrm{rt}},
$$

where $\Phi_{j}^{c}$, and $\Phi_{j}^{\mathrm{rt}}$ stand for the total phase of the $j$ th wave after circular (clockwise or counter-clockwise) rotation and round-trip motion, respectively. The sub-

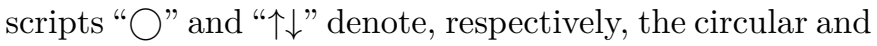
round trip trajectories. The adiabatic geometric phase can, therefore, be viewed as the difference of the actionangle variables in the phase space of $\left(q_{j}^{\prime}, p_{i}^{\prime}\right)[145]$ between the two kinds trajectories. Ref. [61] discussed such circular adiabatic motion of $\boldsymbol{B}$ in its parameter space, around a unit vector with an angle $\Theta$ with the $\Delta \Gamma / 2$ axis (similar to the circular rotation introduced in the undepleted case). Figure 12 manifests the results of the calculation of the AGP through Eq. (47) for the processes of SFG and DFG with different depletion levels.

In the SFG process, the initial condition is generally set as $q_{3}=0$. The geometric phase can then be accumulated both on $\omega_{1}$ and $\omega_{2}$. Now, the relative magnitude of the geometric phases $\gamma_{1}$ and $\gamma_{2}$ (for each of the waves $\omega_{1}$ and $\omega_{2}$ ) is determined based on the initial condition of $I_{j}=\left|q_{j}\right|^{2}$ : if $I_{1}<I_{2}$ then the dominant geometric phase will be accumulated in $\omega_{1}$, and vice versa. We identify two limiting cases: (i) in the undepleted regime, where $I_{1} \ll I_{2}$ (or the other way around), $\omega_{2}$ acts as the pump wave, and the geometric phase is completely accumulated in $\omega_{1}$ (the idler wave); (ii) the fully depleted regime, where $I_{1}=I_{2}$. Here, both waves $\omega_{1}$ and $\omega_{2}$ acquire the same geometric phase, i.e., $\gamma_{1}=\gamma_{2}$. Interestingly, for the case of full depletion, a cusp appears at the north pole of the state surface, making the surface normal $\nabla \varphi$ ill-determined. Hence, the adiabatic approximation breaks down and so does the accumulation of the geometric phase, found to be hindered for $\Theta>0.35 \pi$ [see the green rhombus in Fig. 12(b)]. In the DFG process, the initial condition is generally set as $q_{1}=0$. The adiabatic geometric phase in this case is accumulated only for $\omega_{3}$, in both the undepleted $\left(I_{3} \ll I_{2}\right)$ and fully depleted $\left(I_{3} \gg I_{2}\right)$ regimes. Figure 12 shows the value of the adiabattic geometric phase for different processes and at different regimes, compared with the undepleted result $\gamma=-\pi(1-\cos \Theta)$.

Recently, it was found that Eq. (47) is not only valid for the calculation of the AGP in three-wave mixing, but can also hold for the calculation of the AGP in the process of four-wave mixing [62]. As shown in Fig. 3(b), there are two possible schemes of four wave mixing: Either (I) two photons are annihilated and two new photons are generated, or (II) three photons are annihilated and one new photon is generated. In both schemes, and in a similar manner to what was described above in the case of three 
wave mixing, it is possible to construct canonical Hamilton equations and to map all the possible states of the system onto a geometric surface. For the undepleted case, where it is assumed that there are two undepleted pumps, the surface that describes the two remaining signal and idler waves is simply the Bloch sphere [56, 57], but when the pump depletion becomes significant, the region near one of the poles becomes elongated and attains a conical shape [62], in a similar manner to the case of depleted three wave mixing. There is however one important difference between three wave mixing and four wave mixing: in the latter process and for scheme II, there is an additional contribution to the geometric phase owing to an effective Stark shift, i.e., intensity dependent phase mismatch [62].

\section{Geometric phase in nonlinear metasurfaces}

As we have seen, bulk nonlinear materials provide a versatile and fully-controllable manner to tailor the spatial and spectral shape of the generated light, together with high and robust efficiency of frequency conversion. These concepts have led to the formulation and experimental realization of frequency-domain geometric phases - complementing the $\mathrm{PB}$ phase of light polarization. This new phase can be used for beam shaping and light manipulation schemes employing novel topological phenomena. Most excitingly, the recent advancements in the fabrication of 3D NLPCs [112] offer new avenues for further developments in this field.

However, there are several disadvantages for using bulk nonlinear materials. First, frequency conversion of propagating waves is sensitive to phase-matching conditions. Second, $\chi^{(2)}$ domain engineering is often limited to dimensions larger than the optical wavelength, forbidding wavefront shaping on the sub-wavelength scale. Third, the required length of nonlinear crystals, especially those used in adiabatic frequency conversion, only allows for thick optical elements, which are often less appealing for applications.

\subsection{A brief overview of nonlinear metasurfaces}

For these reasons, the field of nonlinear metasurfaces [11, 155-158] has gained much attention over the recent decade. Such systems allow for ultrathin nonlinear optical components with subwavelength features, that do not rely on phase matching. Their overall low conversion efficiency (reaching at most $10^{-5}$ for all-dielectric metasurfaces [159]) is still unrivaled with bulk nonlinear materials (which, for adiabatic frequency conversion, can even approach 100\%), with the main reason being the low thickness of metasurfaces, allowing only very short interaction lengths. This is somewhat mitigated by the relatively high nonlinear coefficients achievable in nonlinear metasurfaces, realized via optical resonances and field confinement. For example, a giant nonlinear response of $\chi^{(2)}=3 \times 10^{5} \mathrm{pm} / \mathrm{V}$ can be achieved [160], even if the corresponding bulk material can reach values only $3-4$ orders of magnitude smaller. The nonlinear response of nonlinear metasurfaces is determined by the nonlinearity tensor of the constituent meta-atoms, depending on the individual meta-atom geometry, their short- and long-range couplings, and the choice of materials and embedding environment.

Surface nonlinearities are well-known to exist even for materials exhibiting inversion symmetry, which forbids second order nonlinearities $[67,161,162]$ (third-order nonlinearities are, on the other hand, unrestricted by inversion symmetry of bulk materials). Nonlinear metasurfaces are thus commonly constructed from arrays of plasmonic nanoparticles [160, 163-177] (constituting the meta-atoms), where the field is confined to the surface of the meta-atom and its magnitude can be enhanced by matching it with the localized surface plasmon resonance $[163,166]$ and the coupling between metaatoms $[169,174,177]$. When the plasmonic nanoparticles are further coupled to nonlinear materials such as multiple quantum wells or semiconductors $[160,168,172]$ the nonlinearity can dramatically increase. Volume nonlinearities, allowing a larger mode volume and lower heating damage, can also be employed by the use of alldielectric meta-atoms $[156,178,179]$. All these variants of nonlinear metasurfaces were employed for the enhancement and control of various nonlinear processes, such as SHG [160, 163, 165-169, 174, 175, 180, 181], THG [172, 176-179], and FWM [182-184].

While harmonic generation in bulk nonlinear materials is restricted to microscopic selection rules [67], similar rules apply to the macroscopic symmetries of metaatoms $[11,165,175,176]$. For example, if the meta-atom has an $m$-fold rotational symmetry, when a pump with a circular polarization is used, $n$-th harmonic generation is allowed if

$$
n=m l \pm 1,
$$

where $l$ is an integer and the \pm sign stand for the circular polarization of the generated harmonic, being the same ("+") or the opposite ("-") to that of the fundamental wave. Other symmetry-related phenomena, such as circular dichroism [164, 167, 170, 173], were also observed in chiral meta-atoms and meta-molecules.

4.2 Shaping light with nonlinear metasurfaces: Dynamic phase holography

Apart from tailoring the nonlinear response, controlling the geometry and material composition of metasurfaces can be utilized for shaping light beams at the generated harmonics. We associate these methods with the dynamic phase of the nonlinear metasurface response, since they rely on phase differences arising directly from tuning the meta-atom shape and size, or by binary modulation of the 
(a)

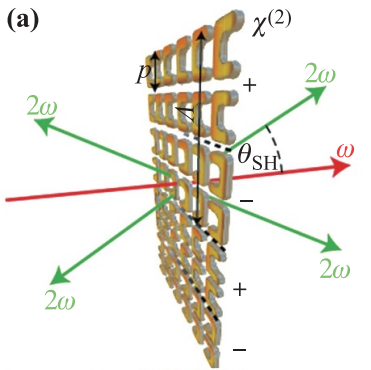

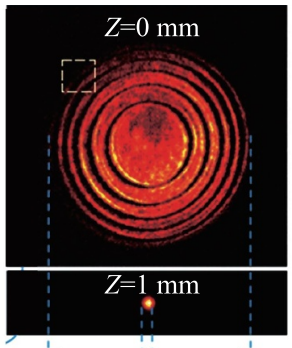

(c)

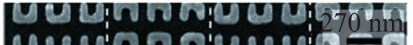

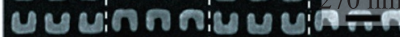

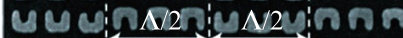

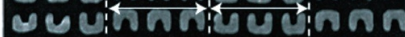
yuvinn

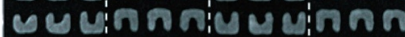
yusinn

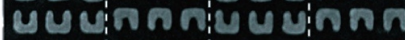
umunnaivusinnn \begin{tabular}{l:c:c:c}
\hline$\chi_{e f f}^{(2)} \uparrow$ & $\downarrow$ & $\uparrow$ & $\downarrow$ \\
\hline
\end{tabular}

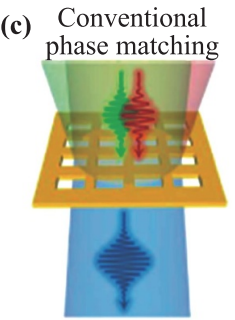

Anomalous phase matching
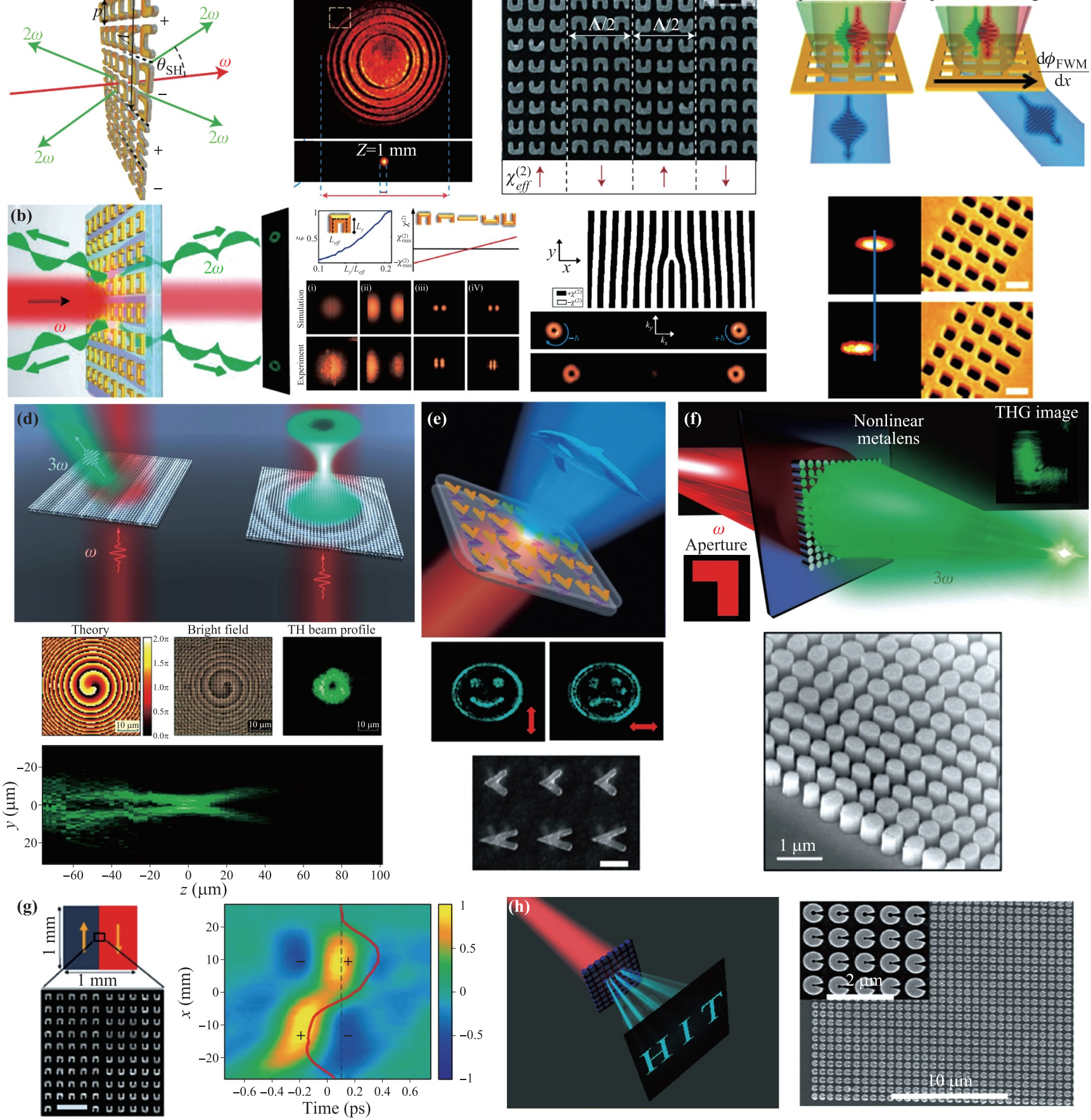

Fig. 12 Beam shaping and dynamic phase holography with nonlinear metasurfaces. (a) Steering and focusing of SHG from nonlinear SRR resonators with modulated transverse phase (modulated SRR orientation). Reproduced from Ref. [146], Copyright $\odot 2015$ Nature Group. (b) Nonlinear shaping of light beams into Laguerre-Gauss and Hermite-Gauss modes using SRR plasmonic metasurfaces with modulated effective nonlinearity (modulated SRR orientation and geometry). Reproduced from Refs. [147, 148]. (c) Steering and focusing of FWM light using nonlinear plasmonic hole arrays with variable rectangular geometry. Reproduced from Ref. [149], Copyright @ 2016 Nature Group. (d) Steering, shaping and focusing of THG from all-dielectric metasurfaces with oval meta-atoms of varying geometry. Reproduced from Ref. [150], Copyright @ 2018 American Chemical Society. (e) Nonlinear holography with double-layered metasurfaces of varying meta-atom geometry, where different holograms are obtained at the THG signal for different input polarizations. Reproduced from Ref. [151], Copyright @ 2016 Nature Group. (f) Nonlinear metasurface lens, allowing for imaging an object at the third harmonic. The lens was fabricated by varying the geometry of oval meta-atoms. Reproduced from Ref. [152], Copyright () 2020 American Chemical Society. (g) Nonlinear spatial and temporal shaping of $\mathrm{THz}$ emission from orientation-modulated SRR nonlinear metasurfaces. Reproduced from Ref. [153], Copyright @ 2019 Nature Group. (h) Nonlinear holography from all-dielectric metasurfaces at the THG signal by varying SRR geometry. Reproduced from Ref. [154], Copyright @ 2018 American Chemical Society. 
metasurface phase pattern. This is done to differentiate these works from the continuous geometric phase holography techniques, which we cover in more detail in the next subsection.

Segal et al. [146], and later Keren-Zur et al. [147] demonstrated beam shaping using binary modulation of plasmonic split-ring resonators (SRR) orientation as well as their shape. Similarly to the control over the nonlinearity in bulk nonlinear materials [Eqs. (13) and (7)], the beam shaping scheme realized in Ref. [147] relied on computergenerated holograms encoded in the structure of the metasurface. The modulated effective $\chi^{(2)}$ coefficient can be written in this case as

$$
\begin{aligned}
\chi_{\text {eff }}^{(2)}(x, y)= & \chi^{(2)} \operatorname{sign}\left\{\cos \left[\frac{2 \pi x}{\Lambda}-\varphi(x, y)\right]\right. \\
& -\cos [\pi q(x, y)]\},
\end{aligned}
$$

where $\varphi(x, y), q(x, y)$ are, respectively, the spacedependent phase and amplitude parameters of the metasurface modulation. Introducing a binary phase $\varphi(x, y)$ can be achieved by varying the orientation of the meatatoms. Controlling the modulation amplitude $q(x, y)$ can be achieved by tailoring the meta-atom shape. This way, it was possible to realize diffractive elements and Fresnel lenses [146] [Fig. 12(a)], as well as shape vortex and Airy beams $[147,148]$ [Fig. 12(b)]. We note that the concept of phase control via orientation of meta-atoms was later on explained also in terms of the nonlinear $\mathrm{PB}$ phase, which we shall introduce below.

Almeida et al. used plasmonic nonlinear metasurfaces of varying meta-atom geometries (rectangular holes in gold) to realize subwavelength phase control [149] [Fig. 12(c)], allowing for realization of anomalous phase matching that results in nonlinear deflection and lensing. A similar idea was proposed by Lin et al., for holographic multiplexing [184]. In another paper [151], Almeida et al. encoded arbitrary holograms by controlling the geometry of L-shaped meta-atoms in a double layer, allowing for different images to be formed at the third harmonic depending on the input polarization [Fig. 12(e)]. Wolf et al. [185] realized optical elements such as beamsplitters in the mid-IR by controlling the shape of gold SRR on top of a quantum-well. Kolkowski et al. [186] demonstrated engineerable chiroptical effects using triangular gold metaatoms. A recent study by Keren-Zur et al. [153] reported the shaping of the spatio-temporal profile of terahertz radiation using binary-modulated gold SRR metasurfaces [Fig. 12(g)].

All-dielectric nonlinear metasurfaces with a varying oval meta-atom shape were employed for generation of vortex beams, deflection and focusing, as demonstrated by Wang et al. [Fig. 12(d)] [150]. Semiconductor dielectric meta-atoms were used to generate vector beams [187]. Gao et al. [154] demonstrated nonlinear holography in the third harmonic, using silicon metasurfaces with varying SRR geometry [Fig. 12(h)]. In a very recent work by Schlickriede et al. [152], the imaging through a nonlinear all-dielectric metasurface lens was demonstrated in the third harmonic, wherein the eccentricity of oval metaatoms was changed to tailor the phase [Fig. 12(f)]. Furthermore, a recent experimental and theoretical study realized full phase control over SHG from AlGaAs, having a very strong nonlinearity albeit anisotropic, using nanochair metatoms, where beam steering and focusing were demonstrated [159].

\subsection{The nonlinear Pancharatnam-Berry phase in meta- surfaces: Physical principles and selection rules}

The phase induced by nonlinear metasurfaces can also be continuously controlled by varying the meta-atom orientation, in a way that is independent from the specific nature of the nonlinear interaction and the meta-atomic resonance, associated with their shape and geometry. This idea can be understood as the generalization of the PB phase to the nonlinear regime. This nonlinear $\mathrm{PB}$ phase is acquired by a simultaneous frequency conversion that may or may not be accompanied by the conversion of the input circular polarization. To explain this mechanism, we adopt the approach suggested by Li et al. [188] and Tymchenko et al. [189], while adding a geometrical interpretation that corresponds to the well-known mechanism of the linear PB phase, introduced in Section 2. We emphasize here that the geometric phase in nonlinear metasurfaces relies on the polarization of light, whereas the geometric phase we discussed for propagating waves in nonlinear materials (Section 3) relies on the frequency of light.

Consider Fig. 13(a), where we depict a u-shaped SRR meta-atom, that is oriented at an angle $\theta$ with respect to the lab transverse coordinate frame $x, y$ (black axes). The local frame of the meta-atom, rotated by this same angle $\theta$ with respect to the lab frame, is then denoted by $x^{\prime}, y^{\prime}$ (blue axes). We first plot the Poincare sphere in the Stokes lab frame $\left(S_{1}, S_{2}, S_{3}\right)$. Suppose the incident light is prepared in a right circular polarization (RCP), so we consider the initial state to be at the north pole of the Poincare sphere. Upon interaction with the metasurface, the light may convert its polarization - corresponding to a trajectory along a great circle traversing the poles.

We then rotate the Poincare sphere to the local Stokes frame $\left(S_{1}^{\prime}, S_{2}^{\prime}, S_{3}^{\prime}\right)$ via a clockwise rotation along $S_{3}$ by an angle $\theta$. In the local frame, the trajectory of the fundamental frequency along the Poincare sphere has an angle $\theta$ with respect to the $S_{1}^{\prime}$ axis. Frequency conversion to the $n$-th harmonic then multiplies this angle by $n$, such that the new angle spanned relative to the $S_{1}^{\prime}$ axis is $n \theta$. The $n$-th harmonic's polarization state is at the south pole (left circular polarization; LCP), and now two outcomes are possible in principal: (i) the polarization state reverts back to the original RCP $(\sigma \rightarrow \sigma)$; (ii) the polarization state stays at the converted LCP, i.e., changes its original sign $(\sigma \rightarrow-\sigma)$. Rotation of the Poincare sphere back to the lab frame will be in opposite direction in each of the 
aforementioned cases: (i) counter-clockwise or (ii) clockwise by an angle $\theta$. For case (i), the polarization state of the $n$-th harmonic traverses a closed trajectory with an angle $(n-1) \theta$ relative to the $S_{1}$ axis, giving a geometric phase of

$$
\gamma=\sigma(n-1) \theta .
$$

In the other case (ii), this angle is $(n+1) \theta$, and the trajectory is not closed. However, as in the linear PB case, the geometric phase can still be defined, and results in

$$
\gamma=\sigma(n+1) \theta .
$$

The same result can be readily obtained through a simple calculation, as was shown in Refs. $[188,189]$. We denote the fundamental harmonic field in the lab frame by $E_{\text {lab }}^{\sigma}$. In the local frame, the field is rotated and given by $E_{\mathrm{loc}}^{\sigma}=$ $E_{\text {lab }}^{\sigma} \mathrm{e}^{\mathrm{i} \sigma \theta}$. The $n$-th nonlinear polarization is thus given by $P_{n, \text { loc }}=\alpha_{0}\left(E_{\text {loc }}^{\sigma}\right)^{n}=\alpha_{0} \mathrm{e}^{\mathrm{i} n \sigma \theta}\left(E_{\text {lab }}^{\sigma}\right)^{n}$, where $\alpha_{0}$ is the local nonlinear polarizability tensor. The rotation back to the lab frame depends on the final polarization state after the interaction, and adds an additional phase factor of $\mathrm{e}^{\mp \mathrm{i} \sigma \theta}$. In terms of the lab frame, we thus find

$$
P_{n, \text { lab }}^{ \pm \sigma}=\alpha_{0} \mathrm{e}^{\mathrm{i} \sigma(n \mp 1) \theta}\left(E_{\text {lab }}^{\sigma}\right)^{n}
$$

giving back Eqs. (50) and (51) for each of the cases above. This property of continuous control over the geometric phase using the orientation of meta-atoms is frequently employed for nonlinear beam shaping and holography, as demonstrated conceptually in Fig. 13(b).

The symmetry properties of meta-atoms determine which nonlinear process takes place in the interaction through Eq. (48). Figure 13(c) depicts the selection rules, and the corresponding geometric phases, generated by meta-atoms with different rotational symmetries. Onefold symmetry allows geometric phase accumulation in both the fundamental, second and third harmonics, with different geometric phases for different changes in polar-

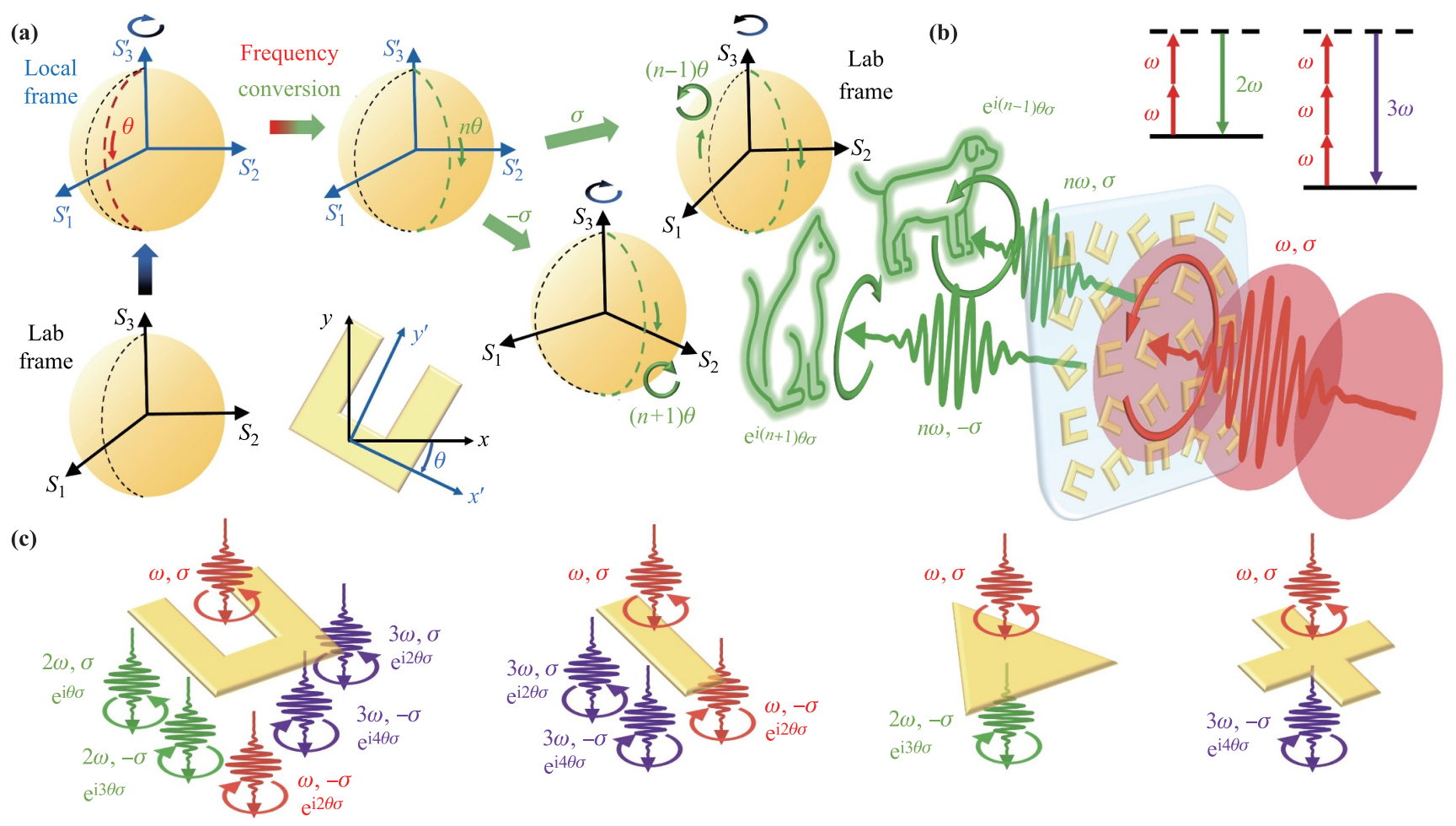

Fig. 13 The nonlinear Pancharatnam-Berry phase. (a) Geometric depiction. A meta-atom fast-axis is rotated by an angle $\theta$ with respect to the lab coordinates. For an incident light of circular polarization $\sigma= \pm 1$ in the fundamental frequency $\omega$, the lab frame Poincare sphere is rotated about $S_{3}$ by an angle $\sigma \theta$ to the local frame. In the local frame, the geodesic trajectory of the fundamental frequency (red dashed curve) spans an angle $\sigma \theta$ with the $\theta=0$ geodesic passing through $S_{1}^{\prime}$ (black dashed curve). Frequency conversion to the $n$-th harmonic increases this angle to $n \sigma \theta$ (rotated geodesic now in green dashed curve). Depending on whether the $n$-th harmonic retains $(\omega, \sigma \rightarrow n \omega, \sigma)$ or flips $(\omega, \sigma \rightarrow n \omega,-\sigma)$ its polarization, the transformation back to the lab frame rotates the local Poincare sphere with an angle $\mp \sigma \theta$ about the $S_{3}^{\prime}$ axis, respectively. The resulting geometric phases in the lab frame are $\gamma_{\sigma, \sigma}=(n-1) \theta \sigma$ and $\gamma_{\sigma,-\sigma}=(n+1) \theta \sigma$. (b) Nonlinear geometric phase holography. The different dependence of the geometric phase on polarization and harmonic order (here depicted second and third harmonic generation, inset) allows to encode different holograms for each output. The geometric phase is encoded through a continuous change in the orientation of the meta-atoms on the meatsurface. (c) Selection rules for different meta-atom symmetry. Left to right: one-, two-, three- and four-fold symmetry, and the possible outcomes in the first, second and third harmonics with and without polarization change. Also noted are the geometric phase values for each case. 
izations. As in bulk materials, SHG is forbidden in metaatoms with inversion symmetry (for example, two- and four-fold symmetries). On the other hand, THG is forbidden for a three-fold symmetry. For two-fold symmetry, the geometric phase is accumulated for both the first and third harmonics. Interestingly, three- and four-fold symmetric meta-atoms do not allow the accumulation of geometric phases in the fundamental harmonic, since their linear response is isotropic. This property can be employed for encoding holograms that are visible only at the converted harmonics [190].

\subsection{Nonlinear Pancharatnam-Berry beam steering, beam shaping and holography}

The concept of thin geometric phase optical elements for beam shaping and holography is well-established in linear optics. In recent years, following the advancements in the field of nonlinear metasurfaces, the concept was borrowed also to the nonlinear realm [11, 201-203].

Some of the earlier works focused on the demonstration of diffractive elements intended to steer light beams according to the generated harmonic and polarization conversion, by inducing a geometric phase gradient in the nonlinear metasurface (Fig. 14). In this respect, Li et al. [188] demonstrated experimentally, and analysed theoretically, the continuous control over the geometric phase for plasmonic metasurfaces with three- and four-fold rotational symmetry [Fig. 14(b)]. In their experiment, the THG signal was diffracted thanks to a geometric phase grating, in a manner that depended on the input polarization. Soon after, the idea of gradient nonlinear metasurfaces using multiple quantum well SRR resonators (onefold symmetry) was analyzed theoretically by Tymchenko et al. [189], where the SHG field was shown to diffract to different directions according to the initial and final po-

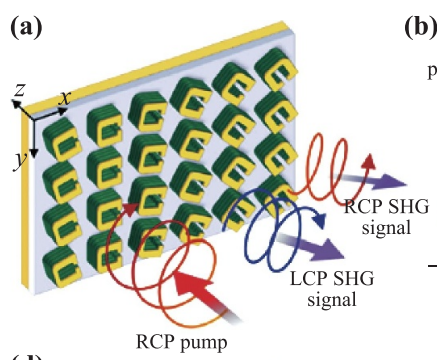

(d)

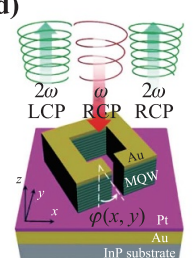

(f)

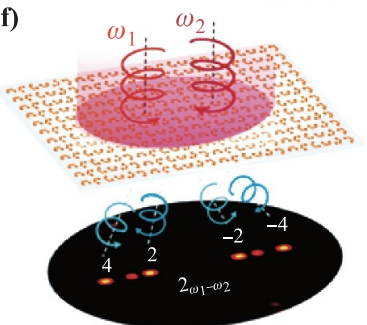

(b) $\mathrm{RCP}$

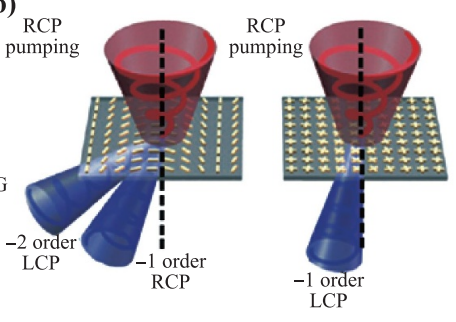

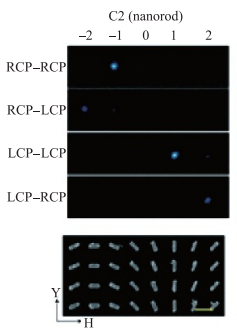

(e)

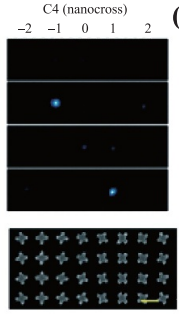

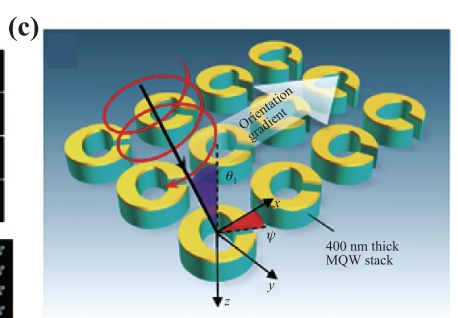
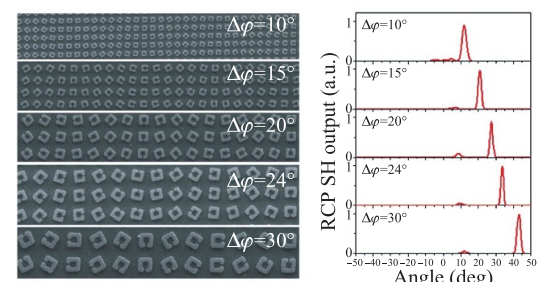

Angle (deg)

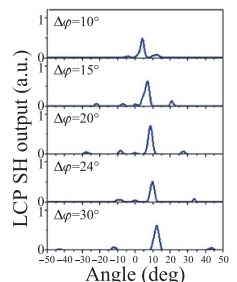

(g)
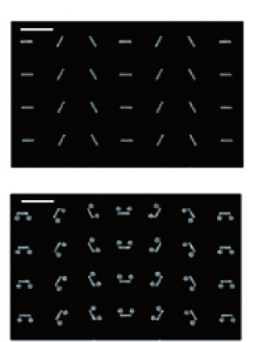

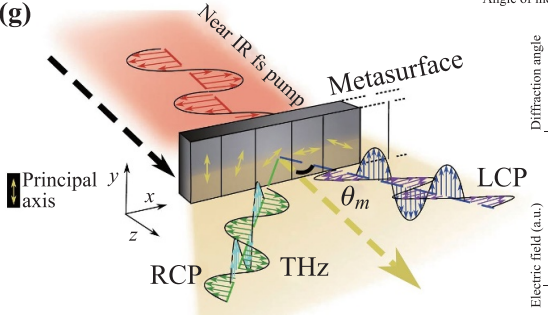

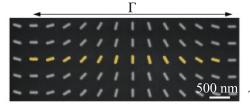
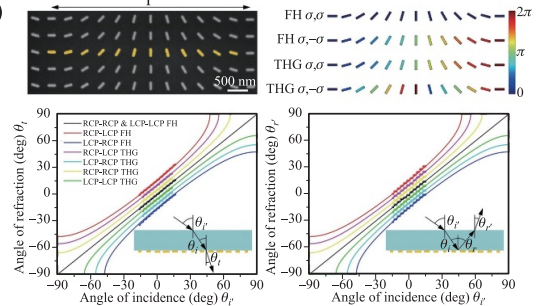
HG $\sigma,-\sigma-\ldots, 1111 \ldots-$
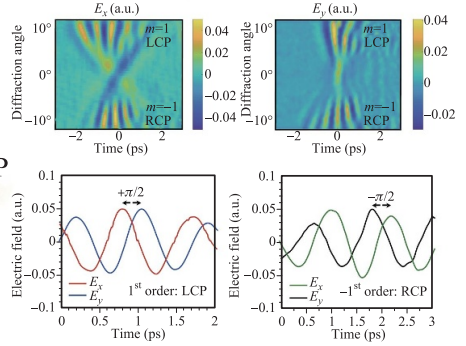

Fig. 14 Nonlinear beam steering using Pacnharatnam-Berry phase gradient metasurfaces. (a) Proposal of gradient nonlinear PB metasurfaces with continuously-varying SRR orientation that diffract the SHG light into different angles, corresonding to the final circular polarization. Reproduced from Ref. [189], Copyright () 2015 American Physical Society. (b) Experimental realiztion of gradient nonlinear PB metasurfaces with two- and four-fold meta-atom symmetry, diffracting the THG signal to differing directions that are correlated with the respective circular polarization change. Reproduced from Ref. [188], Copyright () 2015 Nature Group. (c) Analysis of advanced wavefront control using nonlinear geometric phase metasurfaces. Reproduced from Ref. [191], Copyright () 2016 American Physical Society. (d) Experimental realization of nonlinear PB gradient metasurfaces using gold-on-multiple quantum well SRR meta-atoms having a giant nonlinear response. The meta-atom orientation is varied continuously, resulting in the deflection of SHG light into different angles corresponding to the polarization state. Reproduced from Ref. [160]. (e) Observation of asymmetric nonlinear transmission and refraction of light through PB nonlinear metasurfaces. Reproduced from Ref. [192], Copyright () 2018 American Physical Society. (f) Nonlinear beam steering of FWM light using composite meta-atoms beyond the dipole approximation. Reproduced from Ref. [193], Copyright (C) 2019 American Chemical Society. (g) Nonlinear generation and steering of THz waves from PB metasurfaces with three-fold meta-atom symmetry with a varying orientation. Reproduced from Ref. [194], Copyright () 2021 Nature Group. 
larization, in the presence of a linearly-varying orientation of the meta-atoms [Fig. 14(a)]. Another study by Tymchenko et al. [191] developed the theoretical framework for the design of nonlinear PB metasurfaces, for steering and shaping of light beams [Fig. 14(c)]. Their theory uses an analytic electromagnetic approach for the calculation of the diffracted fields, rather than numerical simulations. An experimental realization of this concept soon followed, where Nookala et al. [160] fabricated a gradient nonlinear metasurfaces comprising gold-on-multiple quantum well SRR meta-atoms with giant nonlinear response, and observed the diffraction of the SHG beam for different polarization and phase gradients [Fig. 14(d)].

Later, Shitrit et al. [192] observed asymmetric light transport induced by nonlinear phase gradient metasurfaces, generalizing Snell's law [Fig. 14(e)]. These con- cepts are not restricted to harmonic generation. Gennaro et al. [193] demonstrated nonlinear diffraction of FWM, finding corrections to the dipole approximation of the $\mathrm{PB}$ phase for composite meta-atoms [Fig. 14(f)]. A recent work by McDonnell et al. [194] demonstrated spatial and temporal shaping of $\mathrm{THz}$ waves generated via DFG (second order optical rectification), using functional nonlinear PB metasurfaces [Fig. 14(g)]. Interestingly, for FWM and DFG, more intricate selection rules arise [193, 194], that are based on the symmetry of the meta-atoms and conservation of spin-angular momentum [176]. In this context, the authors of Ref. [194] used an initial linear polarization for the pump field, since for a pure circular polarization the $\mathrm{THz}$ conversion process was forbidden by the selection rules.

The functionality of nonlinear PB metasurfaces extends (a)

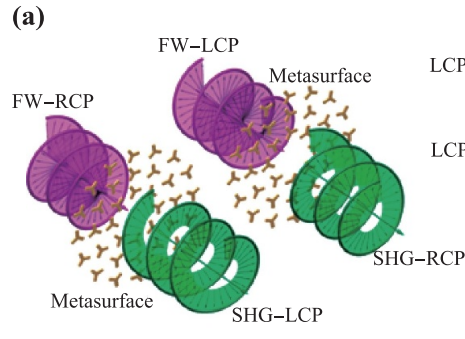

(d)

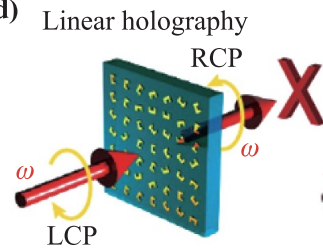

(f)

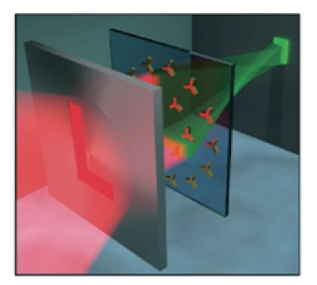

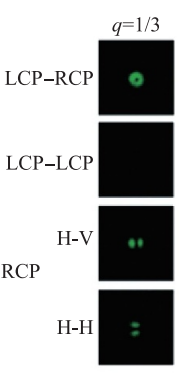
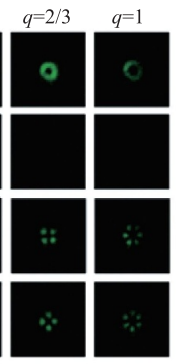

(b)
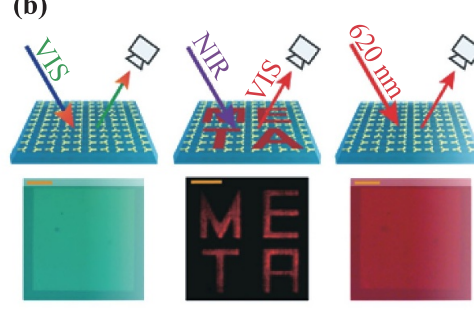

(c)
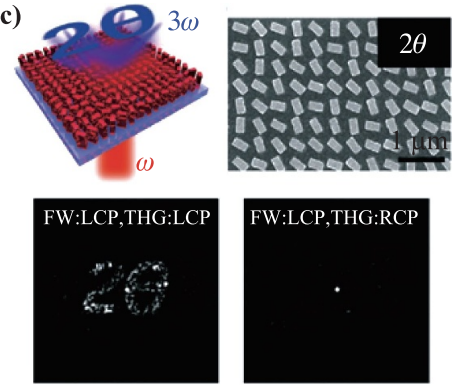

(e)

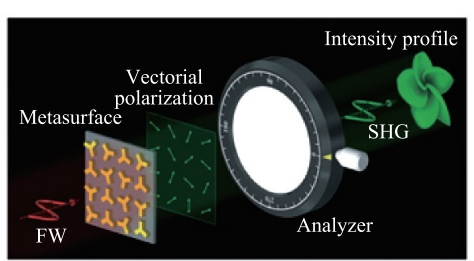

(g)
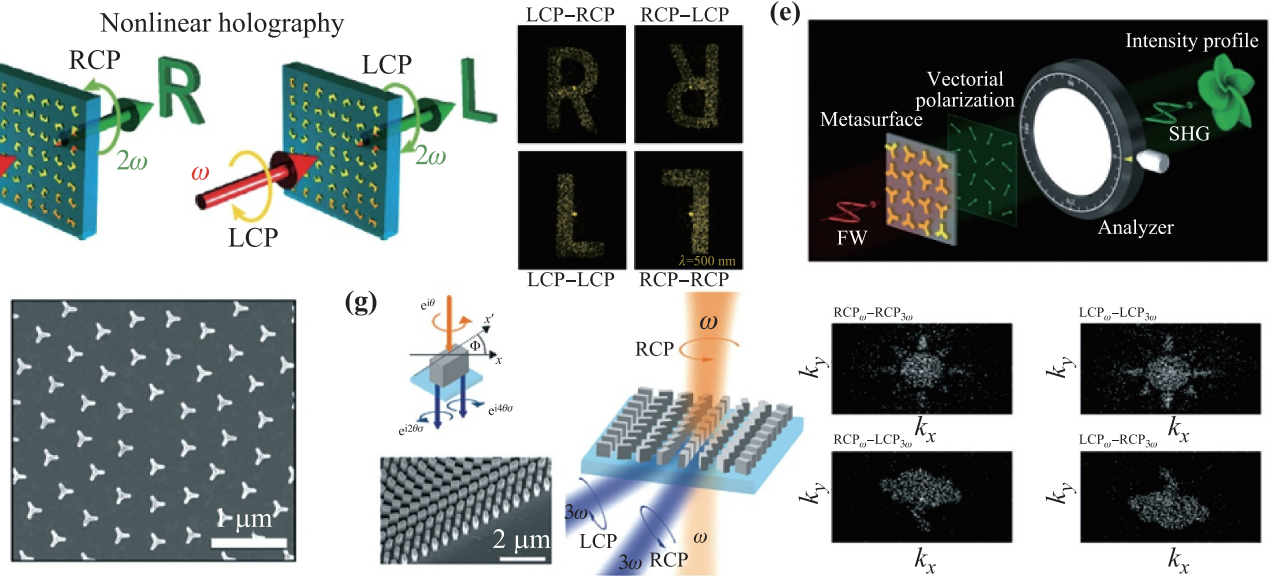

$k_{x}$

$k_{x}$

Fig. 15 Beam shaping and holography with nonlinear PB metasurfaces. (a) Shaping vortex beams at the SHG using nonlinear PB metsurfaces, generalizing the concept of q-plates and spin-orbit couling of light to the nonlinear regime. Reproduced from Ref. [195], Copyright @ 2017 American Chemical Society. (b) Encoding holograms visible only at the SHG, using nonlinear PB metasurfaces with an isotropic linear response (no geometric phase accumulated at the fundamental light). Reproduced from Ref. [190], Copyright $\odot 2017$ American Chemical Society. (c) Holograms and beam steering encoded on all-dielectric nonlinear PB metasurfaces with different meta-atom symmetries. Holograms are visible only for the specific output circular polarization. Reproduced from Ref. [196]. (d) Wavelength and polarization multiplexed holograms, encoded on a nonlinear PB metasurface. The holograms of the letters "X, R, L" are observed at the fundamental, RCP and LCP SHG, respectively. Reproduced from Ref. [197], Copyright @ 2016 Nature Group. (e) Nonlinear hologram encryption on SHG vector beams generated from nonlinear PB metasurfaces. An input fundamental wave with a horizontal polarization is converted to a SHG with a locally-varying linear polarization. After a linear polarizer, the hologram of a flower appears on the screen. Reproduced from Ref. [198], Copyright $\odot$ 2019 American Physical Society. (f) Imaging through a nonlinear PB lens: an object illuminated by an IR fundamental wave is imaged in the SHG signal generated by a nonlinear PB metasurface inducing a quadratic phase. The lens changes between positive and negative as a function of the input circular polarization. Reproduced from Ref. [199]. (g) All-dielectric silicon nonlinear PB metasurfaces for wavefront steering and holography of the THG signal, encoding different holograms for different polarization transitions. Reproduced from Ref. [200], Copyright @ 2019 American Chemical Society. 
beyond diffractive beam steering from geometric phase gradients. Among the effects demonstrated experimentally are nonlinear vortex beam shaping, focusing and imaging through nonlinear PB lenses, and, of course, nonlinear holography. Li et al. [195] designed plasmonic metasurfaces imprinting a vortex phase (i.e., nonlinear q-plates or nonlinear spin-orbit coupling interaction) on a SHG signal [Fig. 15(a)]. The metasurfaces carried different topological charges, inherited by the SHG beam according to the rules Eqs. (48), (50), (51) and Fig. 13(c). The resulting vortex beams were analyzed in the far field for different input polarizations, showing good agreement with simulations. Walter et al. [190] demonstrated holographic image encoding in SHG from plasmonic nonlinear metasurfaces [Fig. 15(b)]. In their experiment, the hologram of the word "META" was visible only at the SHG, since the geometric phase is not accumulated at the fundamental wave (the C3 meta-atom linear response is isotropic).

Liu et al. [196] investigated holography and beam steering of a THG signal from all-dielectric metasurfaces with different meta-atom symmetries [Fig. 15(c)] and realized holograms that are visible only for a certain polarization transition of the THG. Ye et al. [197] multiplexed different holograms on the fundamental and SHG waves of different polarizations, using one-fold symmetric meta-atom plasmonic metasurfaces. Holograms of the letters "X", "R", and "L" were observed for LCP-RCP linear, LCPRCP nonlinear, and LCP-LCP nonlinear, respectively, in the Fourier plane [Fig. 15(d)]. The authors also showed that the interaction is robust and broadband, thanks to the properties of the geometric phase. Tang et al. [198] further extended the context of nonlinear holography to vector beams. By pumping a three-fold symmetric nonlinear metasurface with a horizontally-polarized fundamental wave, the SHG signal results in a linear polarization that is locally rotated by $3 \theta$ according to the orientation of the meta-atoms. After a polarizer is applied to the SHG, a grayscale image with a local intensity of $\cos ^{2} 3 \theta$ is obtained [Fig. 15(e)].

Schlickriede et al. [199] designed a nonlinear metasurface lens for SHG, and observed the imaging of an object illuminated with IR light, where the image is formed by the SHG signal [Fig. 15(f)]. The functionality of the lens is further made unique since for opposite input circular polarization, the focal length is changed from positive to negative. Reineke et al. [200] designed all-dielectric silicon nonlinear PB metasurfaces for wavefront steering and holography of the THG signal, encoding different holograms observed in the THG for different polarization transitions [Fig. 15(g)].

\section{Summary and perspectives}

In this review we presented the recent advancements in the growing field of geometric phase optical elements based on nonlinear frequency conversion. We first covered the physics of the nonlinear interaction of travelling waves in bulk nonlinear photonic crystals, and showed that it can be understood in terms of spin- $1 / 2$ dynamics controlled by external magnetic fields. With this analogy in hand, the realization of both adiabatic and non-adiabatic geometric phases becomes possible in nonlinear frequency conversion. We discussed several theoretical and experimental studies of these systems, with applications ranging from beam shaping, nonreciprocity and all-optical control, to emulation of spin transport phenomena with emergent gauge fields associated with the Berry curvature. The major advantages of using bulk nonlinear media are their high conversion efficiency and robustness thanks to the adiabatic nature of the interaction, and further, that the geometric phase is known to persist even in the fully-nonlinear regime of frequency conversion.

Recent advancements in the fabrication of 3D nonlinear photonic crystals offer exciting new opportunities for the experimental realization of novel effects associated with the geometric phase. As bulk nonlinear crystals can also be employed for shaping and control of quantum light $[106,108,110]$, it is intriguing to consider new directions in which the nonlinear geometric phase could play an important role in shaping and manipulation of down-converted photon pairs in the future.

We then continued to review the fast-growing field of nonlinear Pancharatnam-Berry metasurfaces, that allow the simultaneous nonlinear generation and shaping of light by using ultrathin optical elements with subwavelength phase and amplitude resolution. Their behaviour is dictated by macroscopic selection rules that depend on the rotational symmetry of the constituent meta-atoms, the order of the harmonic generations, and the change in circular polarization. Similar to the linear PB phase, the acquired nonlinear geometric phase depends on the local orientation of the meta-atoms, rather than on their fine-tuned shape - allowing for more robust effects compared to nonlinear beam shaping based on the dynamic phase. Employing these properties, one can steer light beams to different directions, shape various spatial modes, perform nonlinear imaging and multiplex nonlinear holograms, where the functionality is varied according to the generated harmonic order and polarization.

However, the current conversion efficiency of nonlinear metasurfaces is still very low compared to bulk nonlinear photonic crystals. It will be exciting to pursue new materials which exhibit even higher optical nonlinearities in the future, and perhaps combine the concept of $2 \mathrm{D}$ nonlinear PB metasurfaces with 3D phase-matched nonlinear photonic crystals [204]. For example, if future technology allows the fabrication of subwavelength $\chi^{(2)}$ domains, we may expect a new era of nonlinear photonic metamaterials with much higher efficiencies. One possible application of this paradigm could be the generation of entangled photon pairs from metamaterials or highly-efficient nonlinear metasurfaces, a direction that is currently be- 
ing pursued [205-209].

Acknowledgements The authors thank Prof. Eliau Cohen and Prof. Guixin Li for fruitful discussions and comments. A.K. acknowledges support of the Adams Fellowship of the Israeli Academy of Sciences and Humanities. This work was supported by Israel Science Foundation under Grant No. 1415/17.

Open Access This article is licensed under a Creative Commons Attribution 4.0 International License, which permits use, sharing, adaptation, distribution and reproduction in any medium or format, as long as you give appropriate credit to the original author(s) and the source, provide a link to the Creative Commons license, and indicate if changes were made. The images or other third party material in this article are included in the article's Creative Commons license, unless indicated otherwise in a credit line to the material. If material is not included in the article's Creative Commons license and your intended use is not permitted by statutory regulation or exceeds the permitted use, you will need to obtain permission directly from the copyright holder. To view a copy of this license, visit http://creativecommons.org/licenses/by/4.0/.

\section{References}

1. M. V. Berry, Quantal phase factors accompanying adiabatic changes, Proc. R. Soc. A 392(1802), 45 (1984)

2. M. V. Berry, The adiabatic phase and Pancharatnam's phase for polarized light, J. Mod. Opt. 34(11), 1401 (1987)

3. S. M. Rytov, On transition from wave to geometrical optics, Dokl. Akad. Nauk. USSR 18, 263 (1938)

4. V. V. Vladimirsky, The rotation of a polarization plane for curved light ray, Dokl. Akad. Nauk SSSR 31, 222 (1941)

5. S. Pancharatnam, Generalized theory of interference, and its applications, Proc. Indian Acad. Sci. Sect. A 44(5), 247 (1956)

6. M. Berry, Anticipations of the geometric phase, Phys. Today 43(12), 34 (1990)

7. R. Y. Chiao and Y.-S. Wu, Manifestations of Berry's topological phase for the photon, Phys. Rev. Lett. 57(8), 933 (1986)

8. A. Tomita and R. Y. Chiao, Observation of Berry's topological phase by use of an optical fiber, Phys. Rev. Lett. 57(8), 937 (1986)

9. N. Yu and F. Capasso, Flat optics with designer metasurfaces, Nat. Mater. 13, 139 (2014)

10. H. Suchowski, G. Porat, and A. Arie, Adiabatic processes in frequency conversion, Laser Photon. Rev. 8(3), 333 (2014)

11. G. Li, S. Zhang, and T. Zentgraf, Nonlinear photonic metasurfaces, Nat. Rev. Mater. 2(5), 17010 (2017)

12. J. Zak, Berry's phase for energy bands in solids, Phys. Rev. Lett. 62(23), 2747 (1989)

13. J. von Bergmann and H. C. von Bergmann, Foucault pendulum through basic geometry, Am. J. Phys. 75(10), 888 (2007)
14. Y. Zhang, Y. W. Tan, H. L. Stormer, and P. Kim, Experimental observation of the quantum Hall effect and Berry's phase in graphene, Nature 438(7065), 201 (2005)

15. F. D. M. Haldane, Model for a quantum Hall effect without Landau levels: Condensed-matter realization of the "parity anomaly", Phys. Rev. Lett. 61(18), 2015 (1988)

16. B. A. Bernevig, Topological Insulators and Topological Superconductors, Princeton University Press, 2013

17. E. Cohen, H. Larocque, F. Bouchard, F. Nejadsattari, Y. Gefen, and E. Karimi, Geometric phase from AharonovBohm to Pancharatnam-Berry and beyond, Nat. Rev. Phys. 1, 437 (2019)

18. J. Anandan, The geometric phase, Nature 360(6402), 307 (1992)

19. B. Simon, Holonomy, the quantum adiabatic theorem, and Berry's phase, Phys. Rev. Lett. 51(24), 2167 (1983)

20. J. J. Sakurai and J. Napolitano, Modern Quantum Mechanics, Addison-Wesley, 2011

21. Y. Aharonov and J. Anandan, Phase change during a cyclic quantum evolution, Phys. Rev. Lett. 58(16), 1593 (1987)

22. J. Samuel and R. Bhandari, General setting for Berry's phase, Phys. Rev. Lett. 60(23), 2339 (1988)

23. Z. Zhou, Y. Margalit, S. Moukouri, Y. Meir, and R. Folman, An experimental test of the geodesic rule proposition for the noncyclic geometric phase, Sci. Adv. 6(9), eaay8345 (2020)

24. R. Bhandari and J. Samuel, Observation of topological phase by use of a laser interferometer, Phys. Rev. Lett. 60(13), 1211 (1988)

25. T. H. Chyba, R. Simon, L. J. Wang, and L. Mandel, Measurement of the Pancharatnam phase for a light beam, Opt. Lett. 13(7), 562 (1988)

26. Z. Bomzon, G. Biener, V. Kleiner, and E. Hasman, Space-variant Pancharatnam-Berry phase optical elements with computergenerated subwavelength gratings, Optics Lett.27(13), 1141 (2002)

27. E. Hasman, V. Kleiner, G. Biener, and A. Niv, Polarization dependent focusing lens by use of quantized Pancharatnam-Berry phase diffractive optics, Appl. Phys. Lett. 82(3), 328 (2003)

28. X. Ding, F. Monticone, K. Zhang, L. Zhang, D. Gao, S. N. Burokur, A. de Lustrac, Q. Wu, C. W. Qiu, and A. Alù, Ultrathin Pancharatnam-Berry metasurface with maximal cross-polarization efficiency, Adv. Mater. 27(7), 1195 (2015)

29. S. Slussarenko, A. Alberucci, C. P. Jisha, B. Piccirillo, E. Santamato, G. Assanto, and L. Marrucci, Guiding light via geometric phases, Nat. Photon. 10(9), 571 (2016)

30. K. Y. Bliokh, F. J. Rodríguez-Fortuño, F. Nori, and A. V. Zayats, Spin-orbit interactions of light, Nat. Photon. 9, 796 (2015)

31. R. C. Devlin, A. Ambrosio, N. A. Rubin, J. P. B. Mueller, and F. Capasso, Arbitrary spin-to-orbital angular momentum conversion of light, Science 358(6365), 896 (2017) 
32. L. Marrucci, C. Manzo, and D. Paparo, PancharatnamBerry phase optical elements for wave front shaping in the visible domain: Switchable helical mode generation, Appl. Phys. Lett. 88(22), 221102 (2006)

33. G. Biener, A. Niv, V. Kleiner, and E. Hasman, Formation of helical beams by use of Pancharatnam-Berry phase optical elements, Opt. Lett. 27(21), 1875 (2002)

34. F. Cardano, F. Massa, H. Qassim, E. Karimi, S. Slussarenko, D. Paparo, C. De Lisio, F. Sciarrino, E. Santamato, R. W. Boyd, and L. Marrucci, Quantum walks and wavepacket dynamics on a lattice with twisted photons, Sci. Adv. 1(2), e1500087 (2015)

35. J. R. Kurz, A. M. Schober, D. S. Hum, A. J. Saltzman, and M. M. Fejer, Nonlinear physical optics with transversely patterned quasi-phase-matching gratings, IEEE J. Sel. Top. Quantum Electron. 8(3), 660 (2002)

36. A. Shapira, R. Shiloh, I. Juwiler, and A. Arie, Twodimensional nonlinear beam shaping, Opt. Lett. 37(11), 2136 (2012)

37. A. Shapira, I. Juwiler, and A. Arie, Tunable nonlinear beam shaping by non-collinear interactions, Laser Photon. Rev. 7(4), L25 (2013)

38. B. Yang, X. H. Hong, R. E. Lu, Y. Y. Yue, C. Zhang, Y. Q. Qin, and Y. Y. Zhu, 2D wave-front shaping in optical superlattices using nonlinear volume holography, Opt. Lett. 41(13), 2927 (2016)

39. N. V. Bloch, K. Shemer, A. Shapira, R. Shiloh, I. Juwiler, and A. Arie, Twisting light by nonlinear photonic crystals, Phys. Rev. Lett. 108(23), 233902 (2012)

40. S. Trajtenberg-Mills, I. Juwiler, and A. Arie, On-axis shaping of second-harmonic beams, Laser Photon. Rev. 9(6), L40 (2015)

41. Y. Ming, J. Tang, Z.-X. Chen, F. Xu, L.-J. Zhang, and Y.-Q. Lu, Generation of $N 00 N$ state with orbital angular momentum in a twisted nonlinear photonic crystal, IEEE J. Sel. Top. Quantum Electron. 21(3), 225 (2015)

42. T. Ellenbogen, N. Voloch-Bloch, A. Ganany-Padowicz, and A. Arie, Nonlinear generation and manipulation of airy beams, Nat. Photon. 3(7), 395 (2009)

43. J. Imbrock, L. Wesemann, S. Kroesen, M. Ayoub, and C. Denz, Waveguide integrated three-dimensional quasiphase-matching structures, Optica 7(1), 28 (2020)

44. S. Liu, K. Switkowski, C. Xu, J. Tian, B. Wang, P. Lu, W. Krolikowski, and Y. Sheng, Nonlinear wavefront shaping with optically induced three-dimensional nonlinear photonic crystals, Nat. Commun. 10(1), 3208 (2019)

45. P. Mandel, P. Galatola, L. A. Lugiato, and K. G. Wang, Berry phase analogies in nonlinear optics, Opt. Commun. 80(3-4), 262 (1991)

46. M. S. Alber, G. G. Luther, J. E. Marsden, and J. M. Robbins, Geometric phases, reduction and Lie-Poisson structure for the resonant three-wave interaction, Physica D 123(1-4), 271 (1998)

47. V. Y. Toronov and V. L. Derbov, Geometric phases in lasers and liquid flows, Phys. Rev. A 49(2), 1392 (1994)

48. J. C. Garrison and R. Y. Chiao, Geometrical phases from global gauge invariance of nonlinear classical field theories, Phys. Rev. Lett. 60(3), 165 (1988)
49. J. Liu and L. B. Fu, Berry phase in nonlinear systems, Phys. Rev. A 81(5), 052112 (2010)

50. L. D. Zhang, L. B. Fu, and J. Liu, Adiabatic geometric phase in the nonlinear coherent coupler, Eur. Phys. J. D 65(3), 557 (2011)

51. J. Liu, S. C. Li, L. B. Fu, and D. F. Ye, Nonlinear Adiabatic Evolution of Quantum Systems, in: Nonlinear Adiabatic Evolution of Quantum Systems, pp 49-72, Springer Singapore, 2018

52. G. G. Luther, M. S. Alber, J. E. Marsden, and J. M. Robbins, Geometric analysis of optical frequency conversion and its control in quadratic nonlinear media, J. Opt. Soc. Am. B 17(6), 932 (2000)

53. H. Suchowski, D. Oron, A. Arie, and Y. Silberberg, Geometrical representation of sum frequency generation and adiabatic frequency conversion, Phys. Rev. A 78(6), 063821 (2008)

54. H. Suchowski, V. Prabhudesai, D. Oron, A. Arie, and Y. Silberberg, Robust adiabatic sum frequency conversion, Opt. Express 17(15), 12731 (2009)

55. P. Krogen, H. Suchowski, H. Liang, N. Flemens, K. H. Hong, F. X. Kärtner, and J. Moses, Generation and multioctave shaping of mid-infrared intense single-cycle pulses, Nat. Photon. 11(4), 222 (2017)

56. X. Ding, D. Heberle, K. Harrington, N. Flemens, W. Z. Chang, T. A. Birks, and J. Moses, Observation of rapid adiabatic passage in optical four-wave mixing, Phys. Rev. Lett. 124, 153902 (2020)

57. E. Bahar, X. Ding, A. Dahan, H. Suchowski, and J. Moses, Adiabatic four-wave mixing frequency conversion, Opt. Express 26(20), 25582 (2018)

58. K. Wang, Y. Shi, A. S. Solntsev, S. Fan, A. A. Sukhorukov, and D. N. Neshev, Non-reciprocal geometric phase in nonlinear frequency conversion, Opt. Lett. 42(10), 1990 (2017)

59. A. Karnieli and A. Arie, Fully controllable adiabatic geometric phase in nonlinear optics, Opt. Express 26(4), 4920 (2018)

60. A. Karnieli, S. Trajtenberg-Mills, G. Di Domenico, and A. Arie, Experimental observation of the geometric phase in nonlinear frequency conversion, Optica 6(11), 1401 (2019)

61. Y. Li, O. Yesharim, I. Hurvitz, A. Karnieli, S. Fu, G. Porat, and A. Arie, Adiabatic geometric phase in fully nonlinear three-wave mixing, Phys. Rev. A101(3), 033807 (2020)

62. Y. Li, J. Lü, S. Fu, and A. Arie, Geometric representation and the adiabatic geometric phase in four-wave mixing processes, Opt. Express 29(5), 7288 (2021)

63. N. Westerberg, C. Maitland, D. Faccio, K. Wilson, P. Öhberg, and E. M. Wright, Synthetic magnetism for photon fluids, Phys. Rev. A94(2), 023805 (2016)

64. A. Karnieli, S. Tsesses, G. Bartal, and A. Arie, Emulating spin transport with nonlinear optics, from high-order skyrmions to the topological Hall effect, Nat. Commun. 12(1), 1092 (2021)

65. A. Karnieli, S. Tsesses, G. Bartal, and A. Arie, Optical skyrmions and a topological Hall effect in artificial gauge fields, in: CLEO: QELS_Fundamental Science, p. FW4A.6, 2020 
66. D. Smirnova, D. Leykam, Y. Chong, and Y. Kivshar, Nonlinear topological photonics, Appl. Phys. Rev. 7(2), 021306 (2020)

67. R. W. Boyd, Nonlinear Optics, Academic Press, 2008

68. P. D. Maker, R. W. Terhune, M. Nisenoff, and C. M. Savage, Effects of dispersion and focusing on the production of optical harmonics, Phys. Rev. Lett. 8(1), 21 (1962)

69. E. Rozenberg and A. Arie, Broadband and robust adiabatic second harmonic generation by temperature gradient in birefringently phase matched lithium triborate crystal, Opt. Lett. 44(13), 3358 (2019)

70. M. Yamada, N. Nada, M. Saitoh, and K. Watanabe, First-order quasi-phase matched $\mathrm{LiNbO}_{3}$ waveguide periodically poled by applying an external field for efficient blue second-harmonic generation, Appl. Phys. Lett. 62(5), 435 (1993)

71. T. Xu, K. Switkowski, X. Chen, S. Liu, K. Koynov, H. Yu, H. Zhang, J. Wang, Y. Sheng, and W. Krolikowski, Three-dimensional nonlinear photonic crystal in ferroelectric barium calcium titanate, Nat. Photon. 12(10), 591 (2018)

72. D. Wei, C. Wang, H. Wang, X. Hu, D. Wei, X. Fang, Y. Zhang, D. Wu, Y. Hu, J. Li, S. Zhu, and M. Xiao, Experimental demonstration of a three-dimensional lithium niobate nonlinear photonic crystal, Nat. Photon. 12(10), 596 (2018)

73. S. Signorini, M. Mancinelli, M. Borghi, M. Bernard, M. Ghulinyan, G. Pucker, and L. Pavesi, Intermodal fourwave mixing in silicon waveguides, Photon. Res. 6(8), 805 (2018)

74. X. Chen, P. Karpinski, V. Shvedov, K. Koynov, B. Wang, J. Trull, C. Cojocaru, W. Krolikowski, and Y. Sheng, Ferroelectric domain engineering by focused infrared femtosecond pulses, Appl. Phys. Lett. 107(14), 141102 (2015)

75. X. Chen, P. Karpinski, V. Shvedov, A. Boes, A. Mitchell, W. Krolikowski, and Y. Sheng, Quasi-phase matching via femtosecond laser-induced domain inversion in lithium niobate waveguides, Opt. Lett. 41(11), 2410 (2016)

76. S. Keren-Zur and T. Ellenbogen, A new dimension for nonlinear photonic crystals, Nat. Photon. 12, 575 (2018)

77. W. H. Lee, Binary computer-generated holograms, Appl. Opt. 18(21), 3661 (1979)

78. A. Shapira, I. Juwiler, and A. Arie, Nonlinear computergenerated holograms, Opt. Lett. 36(15), 3015 (2011)

79. A. Shapira, L. Naor, and A. Arie, Nonlinear optical holograms for spatial and spectral shaping of light waves, Sci. Bull. (Beijing) 60(16), 1403 (2015)

80. S. Trajtenebrg-Mills and A. Arie, Shaping light beams in nonlinear processes using structured light and patterned crystals, Opt. Mater. Express 7(8), 2928 (2017)

81. G. Imeshev, M. Proctor, and M. M. Fejer, Lateral patterning of nonlinear frequency conversion with radially varying quasi-phase-matching gratings, Opt. Lett. 23(9), 673 (1998)

82. S. M. Saltiel, D. N. Neshev, W. Krolikowski, A. Arie, O. Bang, and Y. S. Kivshar, Multiorder nonlinear diffraction in frequency doubling processes, Opt. Lett. 34(6), 848 (2009)
83. T. Ellenbogen, A. Ganany-Padowicz, and A. Arie, Nonlinear photonic structures for all-optical deflection, Opt. Express 16(5), 3077 (2008)

84. K. Shemer, N. Voloch-Bloch, A. Shapira, A. Libster, I. Juwiler, and A. Arie, Azimuthal and radial shaping of vortex beams generated in twisted nonlinear photonic crystals, Opt. Lett. 38(24), 5470 (2013)

85. B. Zhu, H. Liu, Y. Chen, and X. Chen, High conversion efficiency second-harmonic beam shaping via amplitudetype nonlinear photonic crystals, Opt. Lett. 45(1), 220 (2020)

86. D. Liu, S. Liu, L. M. Mazur, B. Wang, P. Lu, W. Krolikowski, and Y. Sheng, Smart optically induced nonlinear photonic crystals for frequency conversion and control, Appl. Phys. Lett. 116(5), 051104 (2020)

87. B. Zhu, H. Liu, Y. Liu, X. Yan, Y. Chen, and X. Chen, Secondharmonic computer-generated holographic imaging through monolithic lithium niobate crystal by femtosecond laser micromachining, Opt. Lett. 45(15), 4132 (2020)

88. A. Libster-Hershko, S. Trajtenberg-Mills, and A. Arie, Dynamic control of light beams in second harmonic generation, Opt. Lett. 40(9), 1944 (2015)

89. T. Ellenbogen, I. Dolev, and A. Arie, Mode conversion in quadratic nonlinear crystals, Opt. Lett. 33(11), 1207 (2008)

90. Y. Z. Zhi, Y. Li, S. D. Dong, K. J. Yun, W. Zhang, S. Shi, S. S. Bao, and C. G. Guang, Generation of light with controllable spatial patterns via the sum frequency in quasi-phase matching crystals, Sci. Rep. 4(1), 1 (2014)

91. Y. Qin, C. Zhang, Y. Zhu, X. Hu, and G. Zhao, Wavefront engineering by Huygens-Fresnel principle for nonlinear optical interactions in domain engineered structures, Phys. Rev. Lett. 100(6), 063902 (2008)

92. H. H. Xu, B. Yang, C. Zhang, Q. Q. Yi, and Y. Z. Yong, Nonlinear volume holography for wave-front engineering, Phys. Rev. Lett. 113(16), 163902 (2014)

93. I. Dolev, T. Ellenbogen, N. Voloch-Bloch, and A. Arie, Control of free space propagation of Airy beams generated by quadratic nonlinear photonic crystals, Appl. Phys. Lett. 95, 201112 (2009)

94. I. Dolev, A. Libster, and A. Arie, Selfaccelerating parabolic beams in quadratic nonlinear media, Appl. Phys. Lett. 101(10), 101109 (2012)

95. I. Dolev and A. Arie, Three wave mixing of airy beams in a quadratic nonlinear photonic crystals, Appl. Phys. Lett. 97(17), 171102 (2010)

96. I. Dolev, T. Ellenbogen, and A. Arie, Switching the acceleration direction of Airy beams by a nonlinear optical process, Opt. Lett. 35(10), 1581 (2010)

97. I. Dolev, I. Kaminer, A. Shapira, M. Segev, and A. Arie, Experimental observation of self-accelerating beams in quadratic nonlinear media, Phys. Rev. Lett. 108(11), 113903 (2012)

98. S. Trajtenberg-Mills, I. Juwiler, and A. Arie, Generation of second-harmonic beams with switchable curved trajectories, Optica 4(1), 153 (2017) 
99. D. Liu, Y. Zhang, J. Wen, Z. Chen, D. Wei, X. Hu, G. Zhao, S. N. Zhu, and M. Xiao, Diffraction interference induced superfocusing in nonlinear Talbot effect, Sci. Rep. 4, 6134 (2014)

100. G. Imeshev, M. A. Arbore, M. M. Fejer, A. Galvanauskas, M. Fermann, and D. Harter, Ultrashortpulse second-harmonic generation with longitudinally nonuniform quasi-phase-matching gratings: Pulse compression and shaping, J. Opt. Soc. Am. B 17(2), 304 (2000)

101. C. R. Phillips, B. W. Mayer, L. Gallmann, and U. Keller, Frequency-domain nonlinear optics in two-dimensionally patterned quasi-phase-matching media, Opt. Express 24(14), 15940 (2016)

102. R. Shiloh and A. Arie, Spectral and temporal holograms with nonlinear optics, Opt. Lett. 37(17), 3591 (2012)

103. A. Leshem, R. Shiloh, and A. Arie, Experimental realization of spectral shaping using nonlinear optical holograms, Opt. Lett. 39(18), 5370 (2014)

104. R. Remez and A. Arie, Super-narrow frequency conversion, Optica 2(5), 472 (2015)

105. J. P. Torres, A. Alexandrescu, S. Carrasco, and L. Torner, Quasi-phase-matching engineering for spatial control of entangled twophoton states, Opt. Lett. 29(4), 376 (2004)

106. S. Trajtenberg-Mills, A. Karnieli, N. Voloch-Bloch, E. Megidish, H. S. Eisenberg, and A. Arie, Simulating correlations of structured spontaneously down-converted photon pairs, Laser Photon. Rev. 14(3), 1900321 (2020)

107. H. Y. Leng, X. Q. Yu, Y. X. Gong, P. Xu, Z. D. Xie, H. Jin, C. Zhang, and S. N. Zhu, On-chip steering of entangled photons in nonlinear photonic crystals, Nat. Commun. 2(1), 429 (2011)

108. E. Megidish, A. Halevy, H. S. Eisenberg, A. GananyPadowicz, N. Habshoosh, and A. Arie, Compact 2D nonlinear photonic crystal source of beamlike path entangled photons, Opt. Express 21(6), 6689 (2013)

109. L. L. Lu, P. Xu, M. L. Zhong, Y. F. Bai, and S. N. Zhu, Orbital angular momentum entanglement via forkpoling nonlinear photonic crystals, Opt. Express 23(2), 1203 (2015)

110. H. Jin, P. Xu, X. W. Luo, H. Y. Leng, Y. X. Gong, W. J. Yu, M. L. Zhong, G. Zhao, and S. N. Zhu, Compact engineering of path-entangled sources from a monolithic quadratic nonlinear photonic crystal, Phys. Rev. Lett. 111(2), 023603 (2013)

111. A. Bahabad and A. Arie, Generation of optical vortex beams by nonlinear wave mixing, Opt. Express 15(26), 17619 (2007)

112. Y. Zhang, Y. Sheng, S. Zhu, M. Xiao, and W. Krolikowski, Nonlinear photonic crystals: From 2D to 3D, Optica 8(3), 372 (2021)

113. S. Liu, L. M. Mazur, W. Krolikowski, and Y. Sheng, Nonlinear volume holography in 3D nonlinear photonic crystals, Laser Photon. Rev. 14(11), 2000224 (2020)

114. D. Wei, C. Wang, X. Xu, H. Wang, Y. Hu, P. Chen, J. Li, Y. Zhu, C. Xin, X. Hu, Y. Zhang, D. Wu, J. Chu, S. Zhu, and M. Xiao, Efficient nonlinear beam shaping in three-dimensional lithium niobate nonlinear photonic crystals, Nat. Commun. 10(1), 4193 (2019)
115. A. Rangelov and S. Longhi, Nonlinear adiabatic optical isolator, Appl. Opt. 56(11), 2991 (2017)

116. K. Abdelsalam, T. Li, J. B. Khurgin, and S. Fathpour, Linear isolators using wavelength conversion, Optica 7(3), 209 (2020)

117. A. Markov, A. Mazhorova, H. Breitenborn, A. Bruhacs, M. Clerici, D. Modotto, O. Jedrkiewicz, P. di Trapani, A. Major, F. Vidal, and R. Morandotti, Broadband and efficient adiabatic threewave-mixing in a temperaturecontrolled bulk crystal, Opt. Express 26(4), 4448 (2018)

118. T. Ozawa, H. M. Price, A. Amo, N. Goldman, M. Hafezi, L. Lu, M. C. Rechtsman, D. Schuster, J. Simon, O. Zilberberg, and I. Carusotto, Topological photonics, Rev. Mod. Phys. 91(1), 015006 (2019)

119. L. Lu, J. D. Joannopoulos, and M. Soljačić, Topological photonics, Nat. Photon. 8(11), 821 (2014)

120. G. Harari, M. A. Bandres, Y. Lumer, M. C. Rechtsman, Y. D. Chong, M. Khajavikhan, Demetrios N. Christodoulides, and M. Sege, Topological insulator laser: Theory, Science 359(6381), eaar4003 (2018)

121. B. Bahari, A. Ndao, F. Vallini, A. El Amili, Y. Fainman, and B. Kanté, Nonreciprocal lasing in topological cavities of arbitrary geometries, Science 358(6363), 636 (2017)

122. S. Mukherjee and M. C. Rechtsman, Observation of Floquet solitons in a topological bandgap, Science 368(6493), 856 (2020)

123. Y. Wang, L. J. Lang, H. L. Ching, B. Zhang, and Y. D. Chong, Topologically enhanced harmonic generation in a nonlinear transmission line metamaterial, Nat. Commun. 10(1), 1102 (2019)

124. S. Kruk, A. Poddubny, D. Smirnova, L. Wang, A. Slobozhanyuk, A. Shorokhov, I. Kravchenko, B. LutherDavies, and Y. Kivshar, Nonlinear light generation in topological nanostructures, Nat. Nanotechnol. 14(2), 126 (2019)

125. X. Z. Yu, Y. Onose, N. Kanazawa, J. H. Park, J. H. Han, Y. Matsui, N. Nagaosa, and Y. Tokura, Realspace observation of a two-dimensional skyrmion crystal, Nature 465(7300), 901 (2010)

126. A. Fert, N. Reyren, and V. Cros, Magnetic skyrmions: Advances in physics and potential applications, Nat. Rev. Mater. 2(7), 17031 (2017)

127. A. Karnieli and A. Arie, All-optical Stern-Gerlach effect, Phys. Rev. Lett. 120(5), 053901 (2018)

128. A. Karnieli and A. Arie, Frequency domain SternGerlach effect for photonic qubits and qutrits, Optica 5(10), 1297 (2018)

129. P. Bruno, V. K. Dugaev, and M. Taillefumier, Topological Hall effect and Berry phase in magnetic nanostructures, Phys. Rev. Lett. 93(9), 096806 (2004)

130. A. Neubauer, C. Pfleiderer, B. Binz, A. Rosch, R. Ritz, P. G. Niklowitz, and P. Böni, Topological Hall effect in the a phase of MnSi, Phys. Rev. Lett. 102(18), 186602 (2009)

131. N. Kanazawa, Y. Onose, T. Arima, D. Okuyama, K. Ohoyama, S. Wakimoto, K. Kakurai, S. Ishiwata, and Y. Tokura, Large topological Hall effect in a short-period helimagnet MnGe, Phys. Rev. Lett. 106(15), 156603 (2011) 
132. K. Everschor-Sitte and M. Sitte, Realspace Berry phases: Skyrmion soccer, J. Appl. Phys. 115(17), 172602 (2014)

133. J. Ping Liu, Z. Zhang, and G. Zhao, Skyrmions: Topological Structures, Properties, and Applications, CRC Press, 2016

134. G. Porat and A. Arie, Efficient, broadband, and robust frequency conversion by fully nonlinear adiabatic threewave mixing, J. Opt. Soc. Am. B 30(5), 1342 (2013)

135. C. R. Phillips, C. Langrock, D. Chang, Y. W. Lin, L. Gallmann, and M. M. Fejer, Apodization of chirped quasi-phasematching devices, J. Opt. Soc. Am. B 30(6), 1551 (2013)

136. C. R. Phillips and M. M. Fejer, Adiabatic optical parametric oscillators: Steady-state and dynamical behavior, Opt. Express 20(3), 2466 (2012)

137. C. R. Phillips and M. M. Fejer, Efficiency and phase of optical parametric amplification in chirped quasi-phasematched gratings, Opt. Lett. 35(18), 3093 (2010)

138. C. Heese, C. R. Phillips, L. Gallmann, M. M. Fejer, and U. Keller, Ultrabroadband, highly flexible amplifier for ultrashort midinfrared laser pulses based on a periodically poled $\mathrm{Mg}: \mathrm{LiNbO}_{3}$, Opt. Lett. 35(14), 2340 (2010)

139. C. Heese, C. R. Phillips, L. Gallmann, M. M. Fejer, and U. Keller, Role of apodization in optical parametric amplifiers based on aperiodic quasi-phase matching gratings, Opt. Express 20(16), 18066 (2012)

140. C. Heese, C. R. Phillips, B. W. Mayer, L. Gallmann, M. M. Fejer, and U. Keller, $75 \mathrm{MW}$ few-cycle midinfrared pulses from a collinear apodized APPLN-based OPCPA, Opt. Express 20(24), 26888 (2012)

141. O. Yaakobi, L. Caspani, M. Clerici, F. Vidal, and R. Morandotti, Complete energy conversion by autoresonant three-wave mixing in nonuniform media, Opt. Express 21(2), 1623 (2013)

142. O. Yaakobi, M. Clerici, L. Caspani, F. Vidal, and R. Morandotti, Complete pump depletion by autoresonant second harmonic generation in a nonuniform medium, $J$. Opt. Soc. Am. B 30(6), 1637 (2013)

143. J. T. Lü,F. Y. Zhao,W. Pang, and Y. Y. Li, Constant adiabatic geometric phase in three-wave mixing under different depletion levels, Phys. Lett. A 397, 127266 (2021)

144. F. Y. Zhao, J. T. Lü, H. X. He, Y. G. Zhou, S. H. Fu, and Y. Y. Li, Geometric phase with full-wedge and half-wedge rotation in nonlinear frequency conversion, Opt. Express 29(14), 21820 (2021)

145. J. Liu, B. Wu, and Q. Niu, Nonlinear evolution of quantum states in the adiabatic regime, Phys. Rev. Lett. 90(17), 170404 (2003)

146. N. Segal, S. Keren-Zur, N. Hendler, and T. Ellenbogen, Controlling light with metamaterial-based nonlinear photonic crystals, Nat. Photon. 9(3), 180 (2015)

147. S. Keren-Zur, O. Avayu, L. Michaeli, and T. Ellenbogen, Nonlinear beam shaping with plasmonic metasurfaces, ACS Photon. 3(1), 117 (2016)

148. X. L. Gui, M. C. Shu, Y. Cai, S. Zhang, and K. W. Cheah, Third harmonic generation of optical vortices using holography-based Gold-Fork microstructure, Adv. Opt. Mater. 2(4), 389 (2014)
149. E. Almeida, G. Shalem, and Y. Prior, Subwavelength nonlinear phase control and anomalous phase matching in plasmonic metasurfaces, Nat. Commun. 7(1), 10367 (2016)

150. L. Wang, S. Kruk, K. Koshelev, I. Kravchenko, B. LutherDavies, and Y. Kivshar, Nonlinear wavefront control with all-dielectric metasurfaces, Nano Lett. 18(6), 3978 (2018)

151. E. Almeida, O. Bitton, and Y. Prior, Nonlinear metamaterials for holography, Nat. Commun. 7(1), 12533 (2016)

152. C. Schlickriede, S. S. Kruk, L. Wang, B. Sain, Y. Kivshar, and T. Zentgraf, Nonlinear imaging with alldielectric metasurfaces, Nano Lett. 20(6), 4370 (2020)

153. S. Keren-Zur, M. Tal, S. Fleischer, D. M. Mittleman, and T. Ellenbogen, Generation of spatiotemporally tailored terahertz wavepackets by nonlinear metasurfaces, Nat. Commun. 10(1), 1778 (2019)

154. Y. Gao, Y. Fan, Y. Wang, W. Yang, Q. Song, and S. Xiao, Nonlinear holographic all-dielectric metasurfaces, Nano Lett. 18(12), 8054 (2018)

155. A. Krasnok, M. Tymchenko, and A. Alù, Nonlinear metasurfaces: A paradigm shift in nonlinear optics, Mater. Today21(1), 8 (2018)

156. B. Sain, C. Meier, and T. Zentgraf, Nonlinear optics in all-dielectric nanoantennas and metasurfaces: A review, Adv. Photon. 1(02), 1 (2019)

157. S. Keren-Zur, L. Michaeli, H. Suchowski, and T. Ellenbogen, Shaping light with nonlinear metasurfaces, Adv. Opt. Photon. 10(1), 309 (2018)

158. T. Huang, X. Zhao, S. Zeng, A. Crunteanu, P. P. Shum, and N. Yu, Planar nonlinear metasurface optics and their applications, Rep. Prog. Phys. 83(12), 126101 (2020)

159. C. Gigli, G. Marino, A. Artioli, D. Rocco, C. De Angelis, J. Claudon, J. M. Gérard, and G. Leo, Tensorial phase control in nonlinear meta-optics, Optica 8(2), 269 (2021)

160. N. Nookala, J. Lee, M. Tymchenko, J. Sebastian GomezDiaz, F. Demmerle, G. Boehm, K. Lai, G. Shvets, M. C. Amann, A. Alù, and M. Belkin, Ultrathin gradient nonlinear metasurface with a giant nonlinear response, Optica 3(3), 283 (2016)

161. N. Bloembergen and P. S. Pershan, Light waves at the boundary of nonlinear media, Phys. Rev. 128(2), 606 (1962)

162. N. Bloembergen, Surface nonlinear optics: A historical overview, Appl. Phys. B 68(3), 289 (1999)

163. M. W. Klein, C. Enkrich, M. Wegener, and S. Linden, Second-harmonic generation from magnetic metamaterials, Science 313(5786), 502 (2006)

164. S. P. Rodrigues, S. Lan, L. Kang, Y. Cui, and W. Cai, Nonlinear imaging and spectroscopy of chiral metamaterials, Adv. Mater. 26(35), 6157 (2014)

165. R. Hou, V. Shynkar, C. Lafargue, R. Kolkowski, J. Zyss, and F. Lagugné-Labarthet, Second harmonic generation from gold meta-molecules with three-fold symmetry, Phys. Chem. Chem. Phys. 18(11), 7956 (2016)

166. A. Salomon, M. Zielinski, R. Kolkowski, J. Zyss, and Y. Prior, Size and shape resonances in second harmonic generation from silver nanocavities, J. Phys. Chem. C 117(43), 22377 (2013) 
167. V. K. Valev, Characterization of nanostructured plasmonic surfaces with second harmonic generation, Langmuir 28(44), 15454 (2012)

168. J. Lee, M. Tymchenko, C. Argyropoulos, P. Y. Chen, F. Lu, F. Demmerle, G. Boehm, M. C. Amann, A. Alù, and M. A. Belkin, Giant nonlinear response from plasmonic metasurfaces coupled to intersubband transitions, Nature 511(7507), 65 (2014)

169. H. Husu, R. Siikanen, J. Mäkitalo, J. Lehtolahti, J. Laukkanen, M. Kuittinen, and M. Kauranen, Metamaterials with tailored nonlinear optical response, Nano Lett. 12(2), 673 (2012)

170. V. K. Valev, N. Smisdom, A. V. Silhanek, B. De Clercq, W. Gillijns, M. Ameloot, V. V. Moshchalkov, and T. Verbiest, Plasmonic ratchet wheels: Switching circular dichroism by arranging chiral nanostructures, Nano Lett. 9(11), 3945 (2009)

171. K. O'Brien, H. Suchowski, J. Rho, A. Salandrino, B. Kante, X. Yin, and X. Zhang, Predicting nonlinear properties of metamaterials from the linear response, Nat. Mater. 14(4), 379 (2015)

172. H. Aouani, M. Rahmani, M. Navarro-Cía, and S. A. Maier, Third-harmonic upconversion enhancement from a single semiconductor nanoparticle coupled to a plasmonic antenna, Nat. Nanotechnol. 9(4), 290 (2014)

173. M. J. Huttunen, G. Bautista, M. Decker, S. Linden, M. Wegener, and M. Kauranen, Nonlinear chiral imaging of subwavelength-sized twisted-cross gold nanodimers, Opt. Mater. Express 1(1), 46 (2011) (Invited)

174. S. Linden, F. B. P. Niesler, J. Förstner, Y. Grynko, T. Meier, and M. Wegener, Collective effects in secondharmonic generation from splitring-resonator arrays, Phys. Rev. Lett. 109(1), 015502 (2012)

175. K. Konishi, T. Higuchi, J. Li, J. Larsson, S. Ishii, and M. Kuwata-Gonokami, Polarization-controlled circular second harmonic generation from metal hole arrays with threefold rotational symmetry, Phys. Rev. Lett. 112(13), 135502 (2014)

176. S. Chen, G. Li, F. Zeuner, W. H. Wong, Y. B. P. Edwin, T. Zentgraf, K. W. Cheah, and S. Zhang, Symmetry-selective third-harmonic generation from plasmonic metacrystals, Phys. Rev. Lett. 113(3), 033901 (2014)

177. L. Michaeli, S. Keren-Zur, O. Avayu, H. Suchowski, and T. Ellenbogen, Nonlinear surface lattice resonance in plasmonic nanoparticle arrays, Phys. Rev. Lett. 118(24), 243904 (2017)

178. Y. Yang, W. Wang, A. Boulesbaa, I. I. Kravchenko, D. P. Briggs, A. Puretzky, D. Geohegan, and J. Valentine, Nonlinear fano-resonant dielectric metasurfaces, Nano Lett. 15(11), 7388 (2015)

179. M. R. Shcherbakov, D. N. Neshev, B. Hopkins, A. S. Shorokhov, I. Staude, E. V. Melik-Gaykazyan, M. Decker, A. A. Ezhov, A. E. Miroshnichenko, I. Brener, A. A. Fedyanin, and Y. S. Kivshar, Enhanced third-harmonic generation in silicon nanoparticles driven by magnetic response, Nano Lett. 14(11), 6488 (2014)
180. W. Cai, A. P. Vasudev, and M. L. Brongersma, Electrically controlled nonlinear generation of light with plasmonics, Science 333(6050), 1720 (2011)

181. R. Czaplicki, H. Husu, R. Siikanen, J. Mäkitalo, M. Kauranen, J. Laukkanen, J. Lehtolahti, and M. Kuittinen, Enhancement of second-harmonic generation from metal nanoparticles by passive elements, Phys. Rev. Lett. 110(9), 093902 (2013)

182. P. Genevet, J. P. Tetienne, E. Gatzogiannis, R. Blanchard, M. A. Kats, M. O. Scully, and F. Capasso, Large enhancement of nonlinear optical phenomena by plasmonic nanocavity gratings, Nano Lett. 10(12), 4880 (2010)

183. J. Renger, R. Quidant, N. Van Hulst, and L. Novotny, Surface-enhanced nonlinear four-wave mixing, Phys. Rev. Lett. 104(4), 046803 (2010)

184. Z. Lin, L. Huang, T. X. Zhen, X. Li, T. Zentgraf, and Y. Wang, Four-wave mixing holographic multiplexing based on nonlinear metasurfaces, Adv. Opt. Mater. 7(21), 1900782 (2019)

185. O. Wolf, S. Campione, A. Benz, A. P. Ravikumar, S. Liu, T. S. Luk, E. A. Kadlec, E. A. Shaner, J. F. Klem, M. B. Sinclair, and I. Brener, Phased-array sources based on nonlinear metamaterial nanocavities, Nat. Commun. 6(1), 7667 (2015)

186. R. Kolkowski, L. Petti, M. Rippa, C. Lafargue, and J. Zyss, Octupolar plasmonic meta-molecules for nonlinear chiral watermarking at subwavelength scale, ACS Photon. 2(7), 899 (2015)

187. R. Camacho-Morales, M. Rahmani, S. Kruk, L. Wang, L. $\mathrm{Xu}$, D. A. Smirnova, A. S. Solntsev, A. Miroshnichenko, H. H. Tan, F. Karouta, S. Naureen, K. Vora, L. Carletti, C. De Angelis, C. Jagadish, Y. S. Kivshar, and D. N. Neshev, Nonlinear generation of vector beams from AlGaAs nanoantennas, Nano Lett. 16(11), 7191 (2016)

188. G. Li, S. Chen, N. Pholchai, B. Reineke, P. W. H. Wong, E. Y. B. Pun, K. W. Cheah, T. Zentgraf, and S. Zhang, Continuous control of the nonlinearity phase for harmonic generations, Nat. Mater. 14, 607 (2015)

189. M. Tymchenko, J. S. Gomez-Diaz, J. Lee, N. Nookala, M. A. Belkin, and A. Alù, Gradient nonlinear Pancharatnam-Berry metasurfaces, Phys. Rev. Lett. 115(20), 207403 (2015)

190. F. Walter, G. Li, C. Meier, S. Zhang, and T. Zentgraf, Ultrathin nonlinear metasurface for optical image encoding, Nano Lett. 17(5), 3171 (2017)

191. M. Tymchenko, J. S. Gomez-Diaz, J. Lee, N. Nookala, M. A. Belkin, and A. Alù, Advanced control of nonlinear beams with Pancharatnam-Berry metasurfaces, Phys. Rev. B 94(21), 214303 (2016)

192. N. Shitrit, J. Kim, D. S. Barth, H. Ramezani, Y. Wang, and X. Zhang, Asymmetric free-space light transport at nonlinear metasurfaces, Phys. Rev. Lett. 121(4), 046101 (2018)

193. S. D. Gennaro, Y. Li, S. A. Maier, and R. F. Oulton, Nonlinear Pancharatnam-Berry phase metasurfaces beyond the dipole approximation, ACS Photon. 6(9), 2335 (2019) 
194. C. McDonnell, J. Deng, S. Sideris, T. Ellenbogen, and G. Li, Functional THz emitters based on PancharatnamBerry phase nonlinear metasurfaces, Nat. Commun. 12(1), 30 (2021)

195. G. Li, L. Wu, K. F. Li, S. Chen, C. Schlickriede, Z. Xu, S. Huang, W. Li, Y. Liu, E. Y. B. Pun, T. Zentgraf, K. W. Cheah, Y. Luo, and S. Zhang, Nonlinear metasurface for simultaneous control of spin and orbital angular momentum in second harmonic generation, Nano Lett. 17(12), 7974 (2017)

196. B. Liu, B. Sain, B. Reineke, R. Zhao, C. Meier, L. Huang, Y. Jiang, and T. Zentgraf, Nonlinear wavefront control by geometric-phase dielectric metasurfaces: Influence of mode field and rotational symmetry, Adv. Opt. Mater. 8(9), 1902050 (2020)

197. W. Ye, F. Zeuner, X. Li, B. Reineke, S. He, W. Q. Cheng, J. Liu, Y. Wang, S. Zhang, and T. Zentgraf, Spin and wavelength multiplexed nonlinear metasurface holography, Nat. Commun. 7(1), 11930 (2016)

198. Y. Tang, Y. Intaravanne, J. Deng, K. F. Li, X. Chen, and G. Li, Nonlinear vectorial metasurface for optical encryption, Phys. Rev. Appl. 10(2), 024028 (2019)

199. C. Schlickriede, N. Waterman, B. Reineke, P. Georgi, G. $\mathrm{Li}$, S. Zhang, and T. Zentgraf, Imaging through nonlinear metalens using second harmonic generation, Adv. Mater. 30(8), 1703843 (2018)

200. B. Reineke, B. Sain, R. Zhao, L. Carletti, B. Liu, L. Huang, C. De Angelis, and T. Zentgraf, Silicon metasurfaces for third harmonic geometric phase manipulation and multiplexed holography, Nano Lett. 19(9), 6585 (2019)

201. L. Huang, S. Zhang, and T. Zentgraf, Metasurface holography: From fundamentals to applications, Nanophotonics 7(6), 1169 (2018)
202. S. Chen, G. Li, K. W. Cheah, T. Zentgraf, and S. Zhang, Controlling the phase of optical nonlinearity with plasmonic metasurfaces, Nanophotonics 7(6), 1013 (2018)

203. Z. L. Deng and G. Li, Metasurface optical holography, Mater. Today Phys. 3, 16 ( 2017)

204. T. Stolt, J. Kim, S. Héron, A. Vesala, Y. Yang, J. Mun, M. Kim, M. J. Huttunen, R. Czaplicki, M. Kauranen, J. Rho, and P. Genevet, Backward phase-matched secondharmonic generation from stacked metasurfaces, Phys. Rev. Lett. 126(3), 033901 (2021)

205. T. Santiago-Cruz, A. Fedotova, V. Sultanov, M. A. Weissflog, D. Arslan, M. Younesi, T. Pertsch, I. Staude, F. Setzpfandt, and M. Chekhova, Photon pairs from resonant metasurfaces, Nano Lett. 21(10), 4423 (2021)

206. A. S. Solntsev, G. S. Agarwal, and Y. S. Kivshar, Metasurfaces for quantum photonics, Nat. Photon. 15, 327 (2021)

207. C. Okoth, A. Cavanna, T. Santiago-Cruz, and M. V. Chekhova, Microscale generation of entangled photons without momentum conservation, Phys. Rev. Lett. 123(26), 263602 (2019)

208. L. Li, Z. Liu, X. Ren, S. Wang, V.-C. Su, M.-K. Chen, C. H. Chu, H. Y. Kuo, B. Liu, W. Zang, G. Guo, L. Zhang, Z. Wang, S. Zhu, and D. P. Tsai, Metalens-array-based high-dimensional and multiphoton quantum source, Science 368(6498), 1487 (2020)

209. G. Marino, A. S. Solntsev, L. Xu, V. F. Gili, L. Carletti, A. N. Poddubny, M. Rahmani, D. A. Smirnova, H. Chen, A. Lemaître, G. Zhang, A. V. Zayats, C. De Angelis, G. Leo, A. A. Sukhorukov, and D. N. Neshev, Spontaneous photon-pair generation from a dielectric nanoantenna, Optica 6(11), 1416 (2019) 